\title{
An Atom-Economic Inverse Solid-Phase Peptide Synthesis Using Bn or BcM esters of amino acids
}

Jian $\mathrm{Li}^{1,2,3 \dagger}$, Yue Zhu ${ }^{2,4 \dagger}$, Bo Liu' ${ }^{1,2^{*}}$, Feng Tang ${ }^{1,2,3}$, Xing Zheng ${ }^{4}$, Wei Huang ${ }^{1,2,3^{*}}$

${ }^{1}$ School of Pharmaceutical Science and Technology, Hangzhou Institute for Advanced Study, Hangzhou 310024, China. ${ }^{2}$ CAS Key Laboratory of Receptor Research, CAS Center for Excellence in Molecular Cell Science, Shanghai Institute of Materia Medica, Chinese Academy of Sciences, Shanghai 201203, China. 3University of Chinese Academy of Sciences, No.19A Yuquan Road, Beijing 100049, China. ${ }^{4}$ Group of Lead Compound, Department of Pharmacy, Hengyang Medicinal School, University of South China, Hunan Provincial Key Laboratory of Tumor Microenvironment Responsive Drug research, Hunan Province Cooperative Innovation Center for Molecular Target New Drug Study, Hengyang, Hunan, 421001, China. 


\section{Table of Contents}

1. General information $\quad$ S 3

2. Synthesis of building blocks $\quad$ S3

3. General Methods for Peptide Synthesis $\quad$ S13

4. Hydrazinolysis results: Ac-FFGNX-OBn / Ac-FFGNX-NHNH2 S14

$\begin{array}{ll}\text { 5. Survey on the hydrazinolysis efficiency of bulky AAs } & \text { S28 }\end{array}$

6. Research on the extent of racemization $\quad$ S32

7. Evaluation of the efficiency by Fmoc-SPPS V.S. Azide-ISPPS S33

8. Applications of this method S36

9. List of compatible side chain groups in this method S41

10. Copies of ${ }^{1} \mathrm{H}$ and ${ }^{13} \mathrm{C}$ NMR spectra $\quad$ S42 


\section{General information}

\subsection{Reagents}

All commercial materials were used as received without further purification unless otherwise noted. Chemicals were purchased from Sigma Aldrich, J\&K Chemical, Gill Biochemistry or 3A Chemical and were used without further purification. Protected Fmoc-amino acids and coupling reagents were purchased from Shanghai Gill Biochemistry. DCM, DMF, Acetone, Et $2 \mathrm{O}$, EtOAc, piperidine, sodium nitrite, hydrochloric acid, and sodium hydroxide were purchased from Shanghai Sinopharm Chemical Reagent and were used without further purification. 2-Cl-Trt resin $(1.18 \mathrm{mmol} / \mathrm{g})$ and Rink amide AM resin $(0.6 \mathrm{mmol} / \mathrm{g})$ were purchased from Shanghai Gill Biochemistry. ${ }^{1} \mathrm{H}$ NMR spectra were recorded on a Bruker Ascend ${ }^{\mathrm{TM}} 400 \mathrm{MHz}$ or $600 \mathrm{MHz}$ or $500 \mathrm{MHz}$ instrument. Chemical shifts (d) are reported in ppm, and coupling constants $(J)$ are given in $\mathrm{Hz}$.

\subsection{Instruments}

Analytic HPLC analysis was performed on a Beijing ChuangXinTongHeng LC-3000 (analytic model) instrument with a C-18 column $(5 \mu \mathrm{m}, 4.6 \times 150 \mathrm{~mm})$ at $40{ }^{\circ} \mathrm{C}$. Another analytic HPLC analysis was performed on a Chiralcel OJ/OJ-H with a C-18 column $(4.6 \mathrm{~mm} * 150 \mathrm{~mm} * 5 \mathrm{u})$ hand chromatographic column. The column was eluted with a linear gradient of 2-90\% acetonitrile containing $0.1 \%$ TFA for $30 \mathrm{~min}$ at a flow rate of $1 \mathrm{~mL} / \mathrm{min}$. Preparative HPLC was performed on a Beijing Chuang Xin LC-3000 (preparative model) instrument with a C-18 column (10 $\mu \mathrm{m}, 19 \mathrm{x}$ $250 \mathrm{~mm}$ ) at room temperature. The column was eluted with a suitable gradient of 2-90\% acetonitrile containing $0.1 \%$ TFA for $40 \mathrm{~min}$ at a flow rate of $10 \mathrm{~mL} / \mathrm{min}$.

The ESI-MS spectra were measured on an Agilent 6230 LC-TOF MS spectrometer. Key source parameters: drying nitrogen gas flow of $11 \mathrm{~L} / \mathrm{min}$; nebulizer pressure of $40 \mathrm{psi}$; gas temperature of $350{ }^{\circ} \mathrm{C}$; fragmenter voltage of $175 \mathrm{~V}$; skimmer voltage of $65 \mathrm{~V}$; and capillary voltage of $4000 \mathrm{~V}$.

\section{Synthesis of building blocks}

\subsection{Synthesis of $\mathrm{H}-\mathrm{X}_{\mathrm{AA}}-\mathrm{OBn}$}

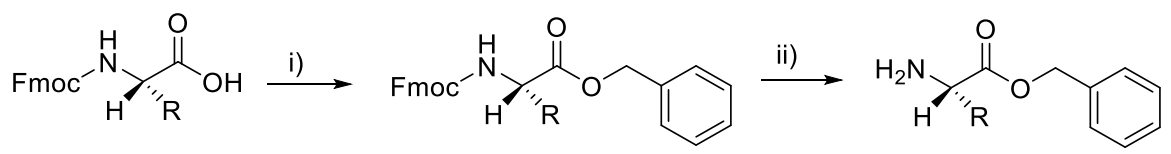

Scheme 1. Synthesis of Amino acid benzyl esters. Reagents and conditions: i) Benzyl bromide, $\mathrm{NaHCO}_{3}$, Aliquate $336, \mathrm{CH}_{2} \mathrm{Cl}_{2}, \mathrm{H}_{2} \mathrm{O}, 11$ h. ii) $20 \%$ Piperidine in DMF.

\section{General Procedure for Preparation of Amino acid benzyl esters ( $\left.\mathrm{H}-\mathrm{X}_{\mathrm{AA}}-\mathrm{OBn}\right)$.}

Fmoc- $\mathrm{X}_{\mathrm{AA}-\mathrm{OH}}$ (1 mmol, 1 equiv.) was stirred in a solution of water and DCM (1:1), which containing $\mathrm{NaHCO}_{3}(1 \mathrm{mmol}, 84 \mathrm{mg}, 1$ equiv), Aliquate 336 ( $1 \mathrm{mmol}, 404 \mathrm{mg}, 1$ equiv.) and benzyl bromide ( $5 \mathrm{mmol}, 593 \mu \mathrm{L}, 5$ equiv.). The reaction was kept under stirring overnight at room temperature. Subsequently, water was added in before transferred to a separating funnel. The aqueous phase was extracted by DCM for 3 times and the combined DCM layer was washed by brine twice afterwards. The organic layer was dried over anhydrous $\mathrm{Na}_{2} \mathrm{SO}_{4}$ and the solvent was removed under reduced pressure. Fmoc- $\mathrm{X}_{\mathrm{AA}}-\mathrm{OBn}$ was purified by flash chromatography (EA/PE, 
1:3) as a white/yellow solidified foam or viscous oil. Then Fmoc- $\mathrm{X}_{\mathrm{AA}}-\mathrm{OBn}$ was dissolved in DCM containing $20 \%$ piperidine. The mixture was stirred under room temperature for $15 \mathrm{~min}$. Finally, organic solvent was concentrated under vacuo and purified by flash column chromatography (EA/PE, 1:1) to give corresponding $\mathrm{H}-\mathrm{X}_{\mathrm{AA}}-\mathrm{OBn}$ (yield 46-88\%).<smiles>CCSCC(N)C(=O)OCc1ccccc1</smiles>

\section{Benzyl S-trityl- $L$-cysteinate}

H-Cys(Trt)-OBn was prepared as a white powder (262.6 mg) in 58\% yield. ${ }^{1} \mathrm{H}$ NMR (500 MHz, Chloroform- $d$ ) $\delta 7.41-7.17$ (m, 20H), 5.07 (s, 2H), 3.14 (dd, $J=8.4,4.3 \mathrm{~Hz}, 1 \mathrm{H}$ ), $2.97(\mathrm{dd}, J=14.1,8.2 \mathrm{~Hz}, 1 \mathrm{H}), 2.81(\mathrm{dd}, J=14.2,4.2 \mathrm{~Hz}, 1 \mathrm{H}) .{ }^{13} \mathrm{C} \mathrm{NMR}\left(126 \mathrm{MHz}, \mathrm{CDCl}_{3}\right) \delta 167.6$, 143.6, 134.1, 129.3, 128.8, 128.6, 128.5, 128.3, 127.2, 68.6, 67.7, 52.1, 31.7 .

ESI-MS, m/z: $[\mathrm{M}+\mathrm{H}]^{+}$calcd for $\mathrm{C}_{29} \mathrm{H}_{28} \mathrm{NO}_{2} \mathrm{~S}^{+} 454.1841$; found 454.1849 .<smiles>NC(Cc1cncn1P)C(=O)OCc1ccccc1</smiles>

\section{Benzyl N $N^{\mathrm{P}}$-trityl- $L$-histidinate}

$\mathrm{H}-\mathrm{His}(\mathrm{Trt})-\mathrm{OBn}$ was prepared as a white powder $(224.5 \mathrm{mg})$ in $46 \%$ yield. ${ }^{1} \mathrm{H}$ NMR $(500 \mathrm{MHz}$, Chloroform- $d$ ) $\delta 7.36(\mathrm{~d}, J=1.4 \mathrm{~Hz}, 1 \mathrm{H}), 7.34-7.07(\mathrm{~m}, 20 \mathrm{H}), 5.18-4.94(\mathrm{~m}$, 2H), 3.90 (dd, $J=7.1,4.6 \mathrm{~Hz}, 1 \mathrm{H}), 3.10-2.80(\mathrm{~m}, 2 \mathrm{H}), 2.42(\mathrm{~s}, 2 \mathrm{H}) .{ }^{13} \mathrm{C}$ NMR $\left(126 \mathrm{MHz}, \mathrm{CDCl}_{3}\right) \delta$ 142.4, 138.7, 135.7, 129.8, 128.5, 128.3, 128.2, 128.1, 119.5, 75.3, 66.7, 54.7, 32.8 .

ESI-MS, m/z: $[\mathrm{M}+\mathrm{H}]^{+}$calcd for $\mathrm{C}_{32} \mathrm{H}_{30} \mathrm{~N}_{3} \mathrm{O}_{2}{ }^{+} 488.2338$; found 488.2342 .<smiles>CSCCC(N)C(=O)OCc1ccccc1</smiles>

\section{Benzyl $L$-methioninate}

H-Met-OBn was prepared as a white powder $(186.6 \mathrm{mg})$ in $78 \%$ yield ${ }^{1} \mathrm{H}$ NMR (500 MHz, Chloroform- $d$ ) $\delta 7.33$ (tdd, $\left.J=6.7,4.9,1.8 \mathrm{~Hz}, 5 \mathrm{H}\right), 5.23(\mathrm{~d}, J=12.1 \mathrm{~Hz}, 1 \mathrm{H})$, $5.16(\mathrm{~d}, J=12.0 \mathrm{~Hz}, 1 \mathrm{H}), 4.20(\mathrm{t}, J=6.2 \mathrm{~Hz}, 1 \mathrm{H}), 2.58-2.57(\mathrm{~m}, 2 \mathrm{H}), 2.31-2.17(\mathrm{~m}, 2 \mathrm{H}), 2.02(\mathrm{~s}$, $3 \mathrm{H}) .{ }^{13} \mathrm{C}$ NMR $\left(126 \mathrm{MHz}, \mathrm{CDCl}_{3}\right) \delta 169.3,134.3,128.9,128.8,128.6,68.5,52.1,29.2,14.8$.

ESI-MS, m/z: $[\mathrm{M}+\mathrm{H}]^{+}$calcd for $\mathrm{C}_{12} \mathrm{H}_{18} \mathrm{NO}_{2} \mathrm{~S}^{+} 240.1058$; found 240.1080 .<smiles>CCNC(=O)C[C@H](N)C(=O)OCc1ccccc1</smiles>

\section{Benzyl N $N^{4}$-trityl- $L$-asparaginate}

$\mathrm{H}-\mathrm{Asn}(\mathrm{Trt})-\mathrm{OBn}$ was prepared as a white powder $(250.7 \mathrm{mg})$ in $54 \%$ yield ${ }^{1} \mathrm{H}$ NMR (500 MHz, Chloroform- $d$ ) $\delta 8.56(\mathrm{~s}, 1 \mathrm{H}), 7.46-7.16(\mathrm{~m}, 20 \mathrm{H}), 5.25-5.08(\mathrm{~m}, 2 \mathrm{H}), 3.90(\mathrm{dd}$, $J=8.9,3.3 \mathrm{~Hz}, 1 \mathrm{H}), 2.83-2.53(\mathrm{~m}, 2 \mathrm{H}) .{ }^{13} \mathrm{C}$ NMR $\left(126 \mathrm{MHz}, \mathrm{CDCl}_{3}\right) \delta 174.0,169.2,144.8,135.3$, 128.7, 128.7, 128.5, 128.3, 127.9, 127.9, 126.9, 70.5, 67.3, 51.6, 40.7.

ESI-MS, m/z: $[\mathrm{M}+\mathrm{H}]^{+}$calcd for $\mathrm{C}_{30} \mathrm{H}_{29} \mathrm{~N}_{2} \mathrm{O}_{3}{ }^{+} 465.2178$; found 465.2174 . 
<smiles>CC(O)C(N)C(=O)OCc1ccccc1</smiles>

\section{Benzyl O-(tert-butyl)- $L$-threoninate}

$\mathrm{H}-\mathrm{Thr}\left({ }^{t} \mathrm{Bu}\right)-\mathrm{OBn}$ was prepared as a white powder $(233.3 \mathrm{mg})$ in $88 \%$ yield ${ }^{1} \mathrm{H}$ NMR (500 MHz, Chloroform-d) $\delta 7.35$ (s, $\left.5 \mathrm{H}\right), 5.27-5.00(\mathrm{~m}, 2 \mathrm{H}), 4.25(\mathrm{qd}, J=6.4,1.9 \mathrm{~Hz}, 1 \mathrm{H})$, $4.02(\mathrm{~d}, J=2.6 \mathrm{~Hz}, 1 \mathrm{H}), 1.33(\mathrm{~d}, J=6.4 \mathrm{~Hz}, 3 \mathrm{H}), 1.07(\mathrm{~s}, 9 \mathrm{H}) .{ }^{13} \mathrm{C}$ NMR $\left(126 \mathrm{MHz}, \mathrm{CDCl}_{3}\right) \delta 168.5$, 134.3, 128.9, 128.8, 128.7, 75.1, 68.4, 65.8, 58.6, 28.2, 20.5.

ESI-MS, m/z: $[\mathrm{M}+\mathrm{H}]^{+}$calcd for $\mathrm{C}_{15} \mathrm{H}_{24} \mathrm{NO}_{3}{ }^{+}$266.1756; found 266.1778 .

\subsection{Synthesis of $\mathrm{H}-\mathrm{X}_{\mathrm{AA}}-\mathrm{OBcM}$}

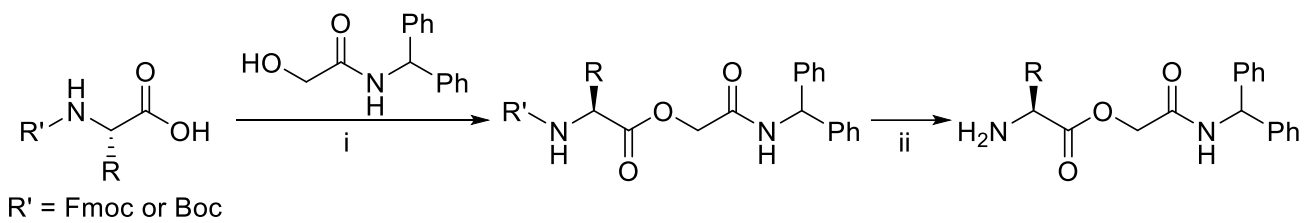

Scheme 2. Synthesis of Amino acid (benzhydryl-carbamoyl)methyl (BcM) ester. Reagents and conditions: i) DCC/DMAP. ii) 20\% piperidine / 50\% TFA in DCM.

\section{General Procedure for Preparation of Amino acid (benzhydryl-carbamoyl)methyl (BcM) ester (H-AA-OBcM)}

(a) Fmoc- $\mathrm{X}_{\mathrm{AA}}-\mathrm{OH}$ (1 mmol, 1 equiv.), N-benzhydryl-2-hydroxyacetamide (1.2mmol, 288mg, 1.2 equiv.), DCC (1.1 mmol, $227 \mathrm{mg}, 1.1$ equiv.) and DMAP (0.1 mmol, $12.2 \mathrm{mg}, 0.1$ equiv.) were added into DCM and stirred until the reaction was finished as monitored by TLC (EA/PE 1:1). The solid was removed by filtration and the filtrate was concentrated to dryness and purified by flash chromatography (EA/PE 1:1) to obtained intermediate, which was dissolved in $16 \mathrm{~mL}$ of a mixture of $\mathrm{CH}_{2} \mathrm{Cl}_{2} /$ Piperidine $(1 / 1, \mathrm{v} / \mathrm{v})$ and stirred at room temperature for $30 \mathrm{~min}$ to remove the Fmoc group. The reaction mixture was concentrated under vacuo and purified by flash column chromatography on silica gel $\left(\mathrm{CH}_{2} \mathrm{Cl}_{2} / \mathrm{MeOH}, 20: 1\right)$ to give corresponding products (yield 30-68\%).

(b) Boc- $\mathrm{X}_{\mathrm{AA}-\mathrm{OH}}$ (1 mmol, 1.0 equiv.), N-benzhydryl-2-hydroxyacetamide (1.2mmol, 288mg, 1.2 equiv.), DCC (1.1 mmol, 227mg, 1.1 equiv.) and DMAP (0.1 mmol, $12.2 \mathrm{mg}, 0.1$ equiv.) were added into DCM and stirred until the reaction was finished as monitored by TLC (EA/PE 1:1). The solid was removed by filtration and the filtrate was concentrated to dryness and purified by flash chromatography to obtained intermediate. Then the residue was subjected with 50\% TFA in DCM to remove the Boc group for 1 hour and evaporated under reduced pressure. The crude compound was purified by flash chromatography (PE/EA 2:1) to get corresponding products (yield 66-89\%). 
<smiles>NCC(=O)OCC(=O)NC(c1ccccc1)c1ccccc1</smiles>

\section{2-(benzhydrylamino)-2-oxoethyl glycinate}

$\mathrm{H}-\mathrm{Gly}-\mathrm{OBcM}$ was prepared as a white powder $(256.3 \mathrm{mg})$ in $86 \%$ yield

${ }^{1} \mathrm{H}$ NMR $\left(500 \mathrm{MHz}\right.$, DMSO- $\left.d_{6}\right) \delta 9.09(\mathrm{~d}, J=8.5 \mathrm{~Hz}, 1 \mathrm{H}), 8.33(\mathrm{~s}, 2 \mathrm{H}), 7.38-7.31(\mathrm{~m}, 4 \mathrm{H}), 7.31$ $-7.23(\mathrm{~m}, 6 \mathrm{H}), 6.14(\mathrm{~d}, J=8.5 \mathrm{~Hz}, 1 \mathrm{H}), 4.78(\mathrm{~s}, 2 \mathrm{H}), 3.93$ (s, 2H). ${ }^{13} \mathrm{C}$ NMR (126 MHz, DMSO$\left.d_{6}\right) \delta 168.0,165.7,142.4,128.9,127.7,127.6,63.7,56.3$.

ESI-MS, m/z: $[\mathrm{M}+\mathrm{H}]^{+}$calcd for $\mathrm{C}_{17} \mathrm{H}_{19} \mathrm{~N}_{2} \mathrm{O}_{3}{ }^{+}$299.1396; found 299.1384 .<smiles>CC(N)C(=O)OCC(=O)NC(c1ccccc1)c1ccccc1</smiles>

\section{2-(benzhydrylamino)-2-oxoethyl $L$-alaninate}

H-Ala-OBcM was prepared as a white powder $(277.7 \mathrm{mg})$ in $89 \%$ yield

${ }^{1} \mathrm{H}$ NMR (500 MHz, DMSO- $\left.d_{6}\right) \delta 9.08(\mathrm{~d}, J=8.5 \mathrm{~Hz}, 1 \mathrm{H}), 8.47(\mathrm{~s}, 2 \mathrm{H}), 7.39-7.31(\mathrm{~m}, 4 \mathrm{H}), 7.31$ $-7.22(\mathrm{~m}, 6 \mathrm{H}), 6.13(\mathrm{~d}, J=8.5 \mathrm{~Hz}, 1 \mathrm{H}), 4.82(\mathrm{~d}, J=14.6 \mathrm{~Hz}, 1 \mathrm{H}), 4.75(\mathrm{~d}, J=14.6 \mathrm{~Hz}, 1 \mathrm{H}), 4.20$ $(\mathrm{q}, J=7.2 \mathrm{~Hz}, 1 \mathrm{H}), 1.44(\mathrm{~d}, J=7.2 \mathrm{~Hz}, 3 \mathrm{H}) .{ }^{13} \mathrm{C}$ NMR $\left(126 \mathrm{MHz}\right.$, DMSO- $\left.d_{6}\right) \delta 170.3,165.6,142.4$, 128.9, 127.7, 127.7, 127.5, 127.5, 63.6, 56.4, 48.2, 16.3.

ESI-MS, m/z: $[\mathrm{M}+\mathrm{H}]^{+}$calcd for $\mathrm{C}_{18} \mathrm{H}_{21} \mathrm{~N}_{2} \mathrm{O}_{3}{ }^{+} 313.1552$; found 313.1547 .

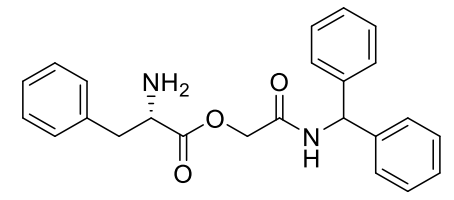

\section{2-(benzhydrylamino)-2-oxoethyl $L$-phenylalaninate}

H-Phe-OBcM was prepared as a light yellow powder $(318.1 \mathrm{mg})$ in $82 \%$ yield ${ }^{1} \mathrm{H}$ NMR $\left(500 \mathrm{MHz}, \mathrm{DMSO}-d_{6}\right) \delta 9.08(\mathrm{~d}, J=8.5 \mathrm{~Hz}, 1 \mathrm{H}), 8.52(\mathrm{~s}, 2 \mathrm{H}), 7.40-7.21(\mathrm{~m}, 15 \mathrm{H}), 6.15$ $(\mathrm{d}, J=8.5 \mathrm{~Hz}, 1 \mathrm{H}), 4.82-4.72(\mathrm{~m}, 2 \mathrm{H}), 4.41(\mathrm{t}, J=6.5 \mathrm{~Hz}, 1 \mathrm{H}), 3.15-3.06(\mathrm{~m}, 2 \mathrm{H}) .{ }^{13} \mathrm{C}$ NMR $(126$ MHz, DMSO- $\left.d_{6}\right) \delta 168.7,165.1,141.9,134.5,129.5,128.5,128.4,127.2,127.2,127.1,63.2,55.9$, 53.0, 35.8 .

ESI-MS, m/z: $[\mathrm{M}+\mathrm{H}]^{+}$calcd for $\mathrm{C}_{24} \mathrm{H}_{25} \mathrm{~N}_{2} \mathrm{O}_{3}{ }^{+} 389.1865$; found 389.1885 .<smiles>CC(C)[C@H](N)C(=O)OCC(=O)NC(c1ccccc1)c1ccccc1</smiles>

\section{2-(benzhydrylamino)-2-oxoethyl $L$-valinate}

H-Val-OBcM was prepared as a white powder $(261.9 \mathrm{mg})$ in $77 \%$ yield.

${ }^{1} \mathrm{H}$ NMR $\left(500 \mathrm{MHz}\right.$, DMSO- $\left.d_{6}\right) \delta 9.08(\mathrm{~d}, J=8.5 \mathrm{~Hz}, 1 \mathrm{H}), 8.41(\mathrm{~s}, 2 \mathrm{H}), 7.41-7.16(\mathrm{~m}, 10 \mathrm{H}), 6.13$ $(\mathrm{d}, J=8.5 \mathrm{~Hz}, 1 \mathrm{H}), 4.81-4.70(\mathrm{~m}, 2 \mathrm{H}), 4.02(\mathrm{~d}, J=4.1 \mathrm{~Hz}, 1 \mathrm{H}), 2.26-2.16(\mathrm{~m}, 1 \mathrm{H}), 1.01-1.04(\mathrm{~m}$, 
6H). ${ }^{13} \mathrm{C}$ NMR (126 MHz, DMSO- $\left.d_{6}\right) \delta 168.7,165.1,142.0,141.99,128.4,128.4,127.2,127.2$, 127.0, 63.2, 57.0, 55.9, 29.4, 17.8, 17.4.

ESI-MS, m/z: $[\mathrm{M}+\mathrm{H}]^{+}$calcd for $\mathrm{C}_{20} \mathrm{H}_{25} \mathrm{~N}_{2} \mathrm{O}_{3} 341.1865$; found 341.1889 .<smiles>CCC(C)[C@H](N)C(=O)OCC(=O)NC(c1ccccc1)c1ccccc1</smiles>

\section{2-(benzhydrylamino)-2-oxoethyl $L$-isoleucinate}

H-Ile-OBcM was prepared as a white powder $(297.5 \mathrm{mg})$ in $84 \%$ yield.

${ }^{1} \mathrm{H}$ NMR $\left(500 \mathrm{MHz}\right.$, DMSO- $\left.d_{6}\right) \delta 9.08(\mathrm{~d}, J=8.5 \mathrm{~Hz}, 1 \mathrm{H}), 8.45(\mathrm{~s}, 2 \mathrm{H}), 7.37-7.22(\mathrm{~m}, 10 \mathrm{H}), 6.13$ $(\mathrm{d}, J=8.5 \mathrm{~Hz}, 1 \mathrm{H}), 4.80-4.76(\mathrm{~m}, 2 \mathrm{H}), 4.07(\mathrm{~d}, J=3.7 \mathrm{~Hz}, 1 \mathrm{H}), 1.96-1.85(\mathrm{~m}, 1 \mathrm{H}), 1.62-1.50$ $(\mathrm{m}, 1 \mathrm{H}), 1.39-1.26(\mathrm{~m}, 1 \mathrm{H}), 0.96(\mathrm{~d}, J=6.9 \mathrm{~Hz}, 3 \mathrm{H}), 0.88(\mathrm{t}, J=7.4 \mathrm{~Hz}, 3 \mathrm{H}) .{ }^{13} \mathrm{C} \mathrm{NMR}(126 \mathrm{MHz}$, DMSO- $\left.d_{6}\right) \delta 168.5,165.1,142.0,142.0,128.4,128.4,127.2,127.2,127.1,127.0,63.2,56.0,55.8$, 36.1, 24.8, 13.9, 11.5 .

ESI-MS, m/z: $[\mathrm{M}+\mathrm{H}]^{+}$calcd for $\mathrm{C}_{21} \mathrm{H}_{27} \mathrm{~N}_{2} \mathrm{O}_{3}{ }^{+}$355.2022; found 355.2017.<smiles>CC(C)CC(N)C(=O)OCC(=O)NC(c1ccccc1)c1ccccc1</smiles>

\section{2-(benzhydrylamino)-2-oxoethyl $L$-leucinate}

H-Leu-OBcM was prepared as a white powder $(286.6 \mathrm{mg})$ in $81 \%$ yield.

${ }^{1} \mathrm{H}$ NMR $\left(500 \mathrm{MHz}\right.$, DMSO- $\left.d_{6}\right) \delta 9.06(\mathrm{~d}, J=8.5 \mathrm{~Hz}, 1 \mathrm{H}), 8.43(\mathrm{~s}, 1 \mathrm{H}), 7.29-7.23(\mathrm{~m}, 2 \mathrm{H}), 6.12(\mathrm{~d}$, $J=8.5 \mathrm{~Hz}, 1 \mathrm{H}), 4.79(\mathrm{q}, J=14.6 \mathrm{~Hz}, 1 \mathrm{H}), 4.06(\mathrm{~d}, J=7.0 \mathrm{~Hz}, 1 \mathrm{H}), 1.88-1.79(\mathrm{~m}, 1 \mathrm{H}), 1.76-$ $1.55(\mathrm{~m}, 1 \mathrm{H}), 0.89(\mathrm{t}, J=3.2 \mathrm{~Hz}, 2 \mathrm{H}) .{ }^{13} \mathrm{C}$ NMR $\left(126 \mathrm{MHz}\right.$, DMSO- $\left.d_{6}\right) \delta 170.2,165.6,142.5,142.5$, 128.9, 127.7, 127.6, 127.6, 127.5, 63.7, 56.4, 50.8, 24.0, 22.6, 22.3.

ESI-MS, m/z: $[\mathrm{M}+\mathrm{H}]^{+}$calcd for $\mathrm{C}_{21} \mathrm{H}_{27} \mathrm{~N}_{2} \mathrm{O}_{3}{ }^{+}$355.2022; found 355.2017.<smiles>NC(CO)C(=O)OCC(=O)NC(c1ccccc1)c1ccccc1</smiles>

\section{2-(benzhydrylamino)-2-oxoethyl $L$-serinate}

H-Ser-OBcM was prepared as a white amorphous solid $(284.2 \mathrm{mg})$ in $74 \%$ yield.

${ }^{1} \mathrm{H}$ NMR $\left(500 \mathrm{MHz}\right.$, DMSO- $\left.d_{6}\right) \delta 9.05(\mathrm{~s}, 1 \mathrm{H}), 8.47(\mathrm{~s}, 1 \mathrm{H}), 7.34(\mathrm{~d}, J=7.3 \mathrm{~Hz}, 1 \mathrm{H}), 7.31-7.23$ $(\mathrm{m}, 4 \mathrm{H}), 6.14(\mathrm{~d}, J=8.5 \mathrm{~Hz}, 1 \mathrm{H}), 5.63(\mathrm{~s}, 1 \mathrm{H}), 4.81(\mathrm{~s}, 1 \mathrm{H}), 4.78(\mathrm{~d}, J=8.7 \mathrm{~Hz}, 1 \mathrm{H}), 4.25(\mathrm{~d}, J=$ $4.3 \mathrm{~Hz}, 1 \mathrm{H}), 3.88(\mathrm{~s}, 1 \mathrm{H}), 3.81(\mathrm{~d}, J=11.7 \mathrm{~Hz}, 1 \mathrm{H}) .{ }^{13} \mathrm{C}$ NMR $\left(126 \mathrm{MHz}, \mathrm{DMSO}-d_{6}\right) \delta 168.3,165.8$, $142.4,142.3,128.9,128.9,127.7,127.7,127.6,127.6,63.8,60.1,56.4,54.7$.

ESI-MS, m/z: $[\mathrm{M}+\mathrm{H}]^{+}$calcd for $\mathrm{C}_{22} \mathrm{H}_{29} \mathrm{~N}_{2} \mathrm{O}_{4}{ }^{+}$385.2127; found 385.2146. 
<smiles>CC(O)[C@H](N)C(=O)OCC(=O)NC(c1ccccc1)c1ccccc1</smiles>

\section{2-(benzhydrylamino)-2-oxoethyl $L$-threoninate}

H-Thr-OBcM was prepared as a white powder $(235.9 \mathrm{mg})$ in $69 \%$ yield.

${ }^{1} \mathrm{H}$ NMR $\left(500 \mathrm{MHz}\right.$, DMSO- $\left.d_{6}\right) \delta 9.07(\mathrm{~d}, J=8.5 \mathrm{~Hz}, 1 \mathrm{H}), 8.36(\mathrm{~s}, 2 \mathrm{H}), 7.38-7.31(\mathrm{~m}, 4 \mathrm{H}), 7.31$ $-7.23(\mathrm{~m}, 6 \mathrm{H}), 6.14(\mathrm{~d}, J=8.5 \mathrm{~Hz}, 1 \mathrm{H}), 5.65(\mathrm{~s}, 1 \mathrm{H}), 4.86-4.76(\mathrm{~m}, 2 \mathrm{H}), 4.21-4.13(\mathrm{~m}, 1 \mathrm{H})$, $4.04(\mathrm{~d}, J=4.3 \mathrm{~Hz}, 1 \mathrm{H}), 1.24(\mathrm{~d}, J=6.5 \mathrm{~Hz}, 3 \mathrm{H}) .{ }^{13} \mathrm{C}$ NMR $\left(126 \mathrm{MHz}, \mathrm{DMSO}-d_{6}\right) \delta 168.4,165.8$, 142.4, 142.3, 128.9, 127.7, 127.6, 65.6, 63.8, 58.2, 56.4, 20.2.

ESI-MS, m/z: $[\mathrm{M}+\mathrm{H}]^{+}$calcd for $\mathrm{C}_{19} \mathrm{H}_{23} \mathrm{~N}_{2} \mathrm{O}_{4}{ }^{+} 343.1658$; found 343.1687 .<smiles>CSCCC(N)C(=O)OCC(=O)NC(c1ccccc1)c1ccccc1</smiles>

\section{2-(benzhydrylamino)-2-oxoethyl $L$-methioninate}

H-Met-OBcM was prepared as a transparent oil $(230 \mathrm{mg})$ in $62 \%$ yield.

${ }^{1} \mathrm{H}$ NMR $\left(500 \mathrm{MHz}, \mathrm{DMSO}-d_{6}\right) \delta 9.10(\mathrm{~d}, J=8.5 \mathrm{~Hz}, 1 \mathrm{H}), 8.53(\mathrm{~s}, 2 \mathrm{H}), 7.38-7.22(\mathrm{~m}, 10 \mathrm{H}), 6.13$ $(\mathrm{d}, J=8.5 \mathrm{~Hz}, 1 \mathrm{H}), 4.81-4.78(\mathrm{~m}, 2 \mathrm{H}), 4.23(\mathrm{t}, J=6.2 \mathrm{~Hz}, 1 \mathrm{H}), 2.66(\mathrm{t}, J=7.7 \mathrm{~Hz}, 2 \mathrm{H}), 2.17-2.04$ $(\mathrm{m}, 2 \mathrm{H}), 2.04(\mathrm{~d}, J=7.7 \mathrm{~Hz}, 3 \mathrm{H}) .{ }^{13} \mathrm{C}$ NMR $\left(126 \mathrm{MHz}, \mathrm{DMSO}-d_{6}\right) \delta 169.0,165.2,142.0,141.9$, 128.4, 127.2, 127.1, 127.1, 127.0, 63.3, 55.9, 50.7, 29.6, 28.0, 14.1 .

ESI-MS, m/z: $[\mathrm{M}+\mathrm{H}]^{+}$calcd for $\mathrm{C}_{2} \mathrm{H}_{25} \mathrm{~N}_{2} \mathrm{O}_{3} \mathrm{~S}^{+} 373.1586$; found 373.1583 .<smiles>CC(C)SC[C@H](N)C(=O)OCC(=O)NC(c1ccccc1)c1ccccc1</smiles>

\section{2-(benzhydrylamino)-2-oxoethyl S-trityl- $L$-cysteinate}

H-Cys(Trt)-OBcM was prepared as a light yellow powder (316.3 $\mathrm{mg}$ ) in 54\% yield.

${ }^{1} \mathrm{H}$ NMR $\left(500 \mathrm{MHz}\right.$, DMSO- $\left.d_{6}\right) \delta 8.88(\mathrm{~d}, J=8.6 \mathrm{~Hz}, 1 \mathrm{H}), 7.33-7.29(\mathrm{~m}, 16 \mathrm{H}), 7.27-7.20(\mathrm{~m}$, 9H), $6.12(\mathrm{~d}, J=8.5 \mathrm{~Hz}, 1 \mathrm{H}), 4.60(\mathrm{~d}, J=14.6 \mathrm{~Hz}, 1 \mathrm{H}), 4.54(\mathrm{~d}, J=14.6 \mathrm{~Hz}, 1 \mathrm{H}), 3.22(\mathrm{t}, J=6.5$ $\mathrm{Hz}, 1 \mathrm{H}), 2.68(\mathrm{~s}, 1 \mathrm{H}), 2.44(\mathrm{~m}, 1 \mathrm{H}), 2.30(\mathrm{dd}, J=12.1,7.1 \mathrm{~Hz}, 1 \mathrm{H}) .{ }^{13} \mathrm{C}$ NMR $(126 \mathrm{MHz}$, DMSO$\left.d_{6}\right) \delta 173.7,166.1,144.8,142.5,129.5,128.8,128.5,127.7,127.5,127.2,66.4,62.9,56.1,54.0$, 38.7, 36.5.

ESI-MS, m/z: $[\mathrm{M}+\mathrm{H}]^{+}$calcd for $\mathrm{C}_{37} \mathrm{H}_{35} \mathrm{~N}_{2} \mathrm{O}_{3} \mathrm{~S}^{+}$587.2368; found 587.2370.<smiles>CC(C)(C)OC(=O)C[C@H](N)C(=O)OCC(=O)NC(c1ccccc1)c1ccccc1</smiles>

1-(2-(benzhydrylamino)-2-oxoethyl) 4-(tert-butyl) $L$-aspartate

H-Asp( $\left.{ }^{(} \mathbf{B u}\right)-\mathbf{O B c M}$ was prepared as a light yellow oil $(173.2 \mathrm{mg})$ in $42 \%$ yield. 
${ }^{1} \mathrm{H}$ NMR $\left(500 \mathrm{MHz}\right.$, DMSO- $\left.d_{6}\right) \delta 8.90(\mathrm{~d}, J=8.5 \mathrm{~Hz}, 1 \mathrm{H}), 7.34-7.23(\mathrm{~m}, 10 \mathrm{H}), 6.16(\mathrm{~d}, J=8.5$ $\mathrm{Hz}, 1 \mathrm{H}), 4.65(\mathrm{~s}, 2 \mathrm{H}), 3.73(\mathrm{t}, J=6.1 \mathrm{~Hz}, 1 \mathrm{H}), 2.62(\mathrm{~m}, 1 \mathrm{H}), 2.48(\mathrm{dd}, J=15.7,7.1 \mathrm{~Hz}, 1 \mathrm{H}), 1.37$ (s, 9H). ${ }^{13} \mathrm{C}$ NMR $\left(126 \mathrm{MHz}\right.$, DMSO- $\left.d_{6}\right) \delta 174.4,170.4,166.4,142.5,128.8,127.7,127.5,80.6$, 62.9, 56.2, 51.5, 40.5, 28.1 .

ESI-MS, m/z: $[\mathrm{M}+\mathrm{H}]^{+}$calcd for $\mathrm{C}_{23} \mathrm{H}_{29} \mathrm{~N}_{2} \mathrm{O}_{5}+413.2076$; found 413.2065 .<smiles>CC(C)(C)OC(=O)CC[C@H](N)C(=O)OCC(=O)NC(c1ccccc1)c1ccccc1</smiles>

\section{1-(2-(benzhydrylamino)-2-oxoethyl) 5-(tert-butyl) $L$-glutamate}

H-Glu( $\left({ }^{t} \mathbf{B u}\right)-\mathbf{O B c M}$ was prepared as a light yellow oil $(132.5 \mathrm{mg})$ in $31 \%$ yield.

${ }^{1} \mathrm{H}$ NMR $\left(500 \mathrm{MHz}\right.$, DMSO- $\left.d_{6}\right) \delta 9.11(\mathrm{~d}, J=8.5 \mathrm{~Hz}, 1 \mathrm{H}), 8.46(\mathrm{~s}, 2 \mathrm{H}), 7.38-7.31(\mathrm{~m}, 4 \mathrm{H}), 7.27$ $(\mathrm{dd}, J=14.0,7.1 \mathrm{~Hz}, 6 \mathrm{H}), 6.12(\mathrm{~d}, J=8.5 \mathrm{~Hz}, 1 \mathrm{H}), 4.80-4.78(\mathrm{~m}, 2 \mathrm{H}), 4.18(\mathrm{~s}, 1 \mathrm{H}), 2.50-2.46(\mathrm{~m}$, $2 \mathrm{H}), 2.10-1.96(\mathrm{~m}, 2 \mathrm{H}), 1.39(\mathrm{~s}, 9 \mathrm{H}) .{ }^{13} \mathrm{C}$ NMR $\left(126 \mathrm{MHz}, \mathrm{DMSO}-d_{6}\right) \delta 171.5,169.5,165.7,142.5$, $142.4,128.9,127.7,127.6,127.6,127.6,80.6,63.8,56.3,51.4,30.2,28.2,25.8$.

ESI-MS, m/z: $[\mathrm{M}+\mathrm{H}]^{+}$calcd for $\mathrm{C}_{24} \mathrm{H}_{31} \mathrm{~N}_{2} \mathrm{O}_{5}{ }^{+} 427.2233$; found 427.2253 .

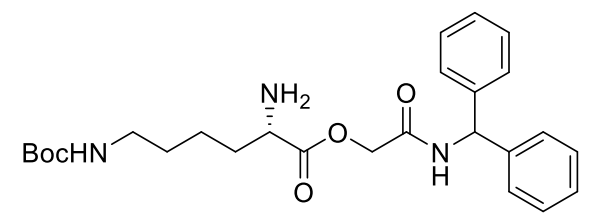

\section{2-(benzhydrylamino)-2-oxoethyl $\mathbf{N}^{6}$-(tert-butoxycarbonyl)- $L$-lysinate}

H-Lys (Boc)-OBcM was prepared as a white powder (230.4 $\mathrm{mg}$ ) in $49 \%$ yield. ${ }^{1} \mathrm{H}$ NMR $\left(500 \mathrm{MHz}\right.$, DMSO- $\left.d_{6}\right) \delta 8.96(\mathrm{~d}, J=8.6 \mathrm{~Hz}, 1 \mathrm{H}), 7.36-7.25(\mathrm{~m}, 11 \mathrm{H}), 6.78(\mathrm{t}, J=5.5$ $\mathrm{Hz}, 1 \mathrm{H}), 6.13(\mathrm{~d}, J=8.5 \mathrm{~Hz}, 1 \mathrm{H}), 4.62(\mathrm{q}, J=14.6 \mathrm{~Hz}, 2 \mathrm{H}), 3.36(\mathrm{~m}, 3 \mathrm{H}), 2.87(\mathrm{~d}, J=5.7 \mathrm{~Hz}, 2 \mathrm{H})$, $1.66-1.40(\mathrm{~m}, 2 \mathrm{H}), 1.36(\mathrm{~s}, 9 \mathrm{H}), 1.34-1.30(\mathrm{~m}, 2 \mathrm{H}) .{ }^{13} \mathrm{C}$ NMR $\left(126 \mathrm{MHz}\right.$, DMSO- $\left.d_{6}\right) \delta 175.8$, 171.5, 166.4, 156.0, 142.7, 142.6, 142.5, 128.8, 127.7, 127.7, 127.5, 127.4, 77.7, 62.6, 61.9, 56.1, $55.9,54.2,34.5,29.7,28.7,22.9$.

ESI-MS, m/z: $[\mathrm{M}+\mathrm{H}]^{+}$calcd for $\mathrm{C}_{26} \mathrm{H}_{36} \mathrm{~N}_{3} \mathrm{O}_{5}{ }^{+} 470.2655$; found 470.2686 .<smiles>N[C@@H]1Cc2cncn2C1C(=O)OCC(=O)NC(c1ccccc1)c1ccccc1</smiles>

\section{2-(benzhydrylamino)-2-oxoethyl $\mathrm{N}^{\mathrm{P}}$-trityl- $L$-histidinate}

H-His(Trt)-OBcM was prepared as a white powder (229.8 $\mathrm{mg}$ ) in 37\% yield.

${ }^{1} \mathrm{H}$ NMR $\left(500 \mathrm{MHz}\right.$, DMSO- $\left.d_{6}\right) \delta 9.63(\mathrm{~d}, J=8.6 \mathrm{~Hz}, 1 \mathrm{H}), 7.39-7.31(\mathrm{~m}, 2 \mathrm{H}), 7.22(\mathrm{~d}, J=7.5 \mathrm{~Hz}$, $1 \mathrm{H}), 7.18(\mathrm{t}, J=7.5 \mathrm{~Hz}, 1 \mathrm{H}), 7.15-7.09(\mathrm{~m}, 3 \mathrm{H}), 6.97(\mathrm{dd}, J=5.2,1.7 \mathrm{~Hz}, 3 \mathrm{H}), 6.69(\mathrm{~s}, 1 \mathrm{H}), 6.55$ $(\mathrm{d}, J=1.3 \mathrm{~Hz}, 1 \mathrm{H}), 6.20(\mathrm{~d}, J=8.6 \mathrm{~Hz}, 1 \mathrm{H}), 4.71(\mathrm{~d}, J=15.1 \mathrm{~Hz}, 1 \mathrm{H}), 4.52(\mathrm{~d}, J=15.1 \mathrm{~Hz}, 1 \mathrm{H})$, $3.64(\mathrm{~d}, J=5.4 \mathrm{~Hz}, 1 \mathrm{H}), 2.91-2.83(\mathrm{~m}, 1 \mathrm{H}), 2.76(\mathrm{~m}, 1 \mathrm{H}) .{ }^{13} \mathrm{C}$ NMR $\left(126 \mathrm{MHz}\right.$, DMSO- $\left.d_{6}\right) \delta$ $175.1,167.5,142.5,142.1,142.1,138.4,136.8,129.6,128.7,128.6,128.4,127.9,127.8,127.5$, $127.4,119.9,74.8,63.0,55.7,54.5,33.0$.

ESI-MS, m/z: $[\mathrm{M}+\mathrm{H}]^{+}$calcd for $\mathrm{C}_{40} \mathrm{H}_{37} \mathrm{~N}_{4} \mathrm{O}_{3}{ }^{+} 621.2866$; found 621.2886 . 
<smiles>CCNC(=O)C[C@H](N)C(=O)OCC(=O)NC(c1ccccc1)c1ccccc1</smiles>

\section{2-(benzhydrylamino)-2-oxoethyl $N^{4}$-trityl- $L$-asparaginate}

H-Asn(Trt)-OBcM was prepared as a white powder $(388.7 \mathrm{mg})$ in $65 \%$ yield.

${ }^{1} \mathrm{H}$ NMR $\left(500 \mathrm{MHz}\right.$, DMSO- $\left.d_{6}\right) \delta 9.17(\mathrm{~s}, 1 \mathrm{H}), 8.95(\mathrm{~d}, J=8.6 \mathrm{~Hz}, 1 \mathrm{H}), 7.34-7.28(\mathrm{~m}, 4 \mathrm{H}), 7.28$ $-7.23(\mathrm{~m}, 12 \mathrm{H}), 7.21-7.15(\mathrm{~m}, 9 \mathrm{H}), 6.12(\mathrm{~d}, J=8.6 \mathrm{~Hz}, 1 \mathrm{H}), 4.68-4.56(\mathrm{~m}, 2 \mathrm{H}), 3.73(\mathrm{dd}, J=$ 7.4, $5.2 \mathrm{~Hz}, 1 \mathrm{H}), 2.63-2.56(\mathrm{~m}, 2 \mathrm{H}) .{ }^{13} \mathrm{C}$ NMR $\left(126 \mathrm{MHz}, \mathrm{DMSO}-d_{6}\right) \delta 174.7,169.9,166.4,145.2$, $142.5,128.9,128.8,128.8,127.9,127.7,127.5,126.8,69.8,62.8,56.2,51.6,21.2$

ESI-MS, m/z: $[\mathrm{M}+\mathrm{H}]^{+}$calcd for $\mathrm{C}_{38} \mathrm{H}_{36} \mathrm{~N}_{3} \mathrm{O}_{4}{ }^{+}$598.2706; found 598.2718.<smiles>CCNC(=O)CCC(N)C(=O)OCC(=O)NC(c1ccccc1)c1ccccc1</smiles>

\section{2-(benzhydrylamino)-2-oxoethyl $\mathrm{N}^{5}$-trityl- $L$-glutaminate}

H-GIn(Trt)-OBcM was prepared as a white powder $(416.2 \mathrm{mg})$ in $68 \%$ yield.

${ }^{1} \mathrm{H}$ NMR $\left(500 \mathrm{MHz}\right.$, DMSO- $\left.d_{6}\right) \delta 9.10(\mathrm{~d}, J=8.5 \mathrm{~Hz}, 1 \mathrm{H}), 8.66(\mathrm{~s}, 1 \mathrm{H}), 8.42(\mathrm{~s}, 2 \mathrm{H}), 7.48-7.08$ $(\mathrm{m}, 25 \mathrm{H}), 6.10(\mathrm{~d}, J=8.5 \mathrm{~Hz}, 1 \mathrm{H}), 4.80-4.78(\mathrm{~m}, 2 \mathrm{H}), 4.01(\mathrm{~s}, 1 \mathrm{H}), 2.55(\mathrm{dd}, J=11.3,5.6 \mathrm{~Hz}, 2 \mathrm{H})$, 1.98-1.96 (m, 2H). ${ }^{13} \mathrm{C}$ NMR (126 MHz, DMSO- $\left.d_{6}\right) \delta 170.7,169.1,165.2,144.2,141.9,141.9$, 128.4, 128.4, 127.4, 127.2, 127.2, 127.1, 127.1, 126.3, 69.3, 63.2, 55.9, 51.3, 31.1, 26.2.

ESI-MS, m/z: $[\mathrm{M}+\mathrm{H}]^{+}$calcd for $\mathrm{C}_{39} \mathrm{H}_{38} \mathrm{~N}_{3} \mathrm{O}_{4}{ }^{+}$612.2862; found 612.2886 .

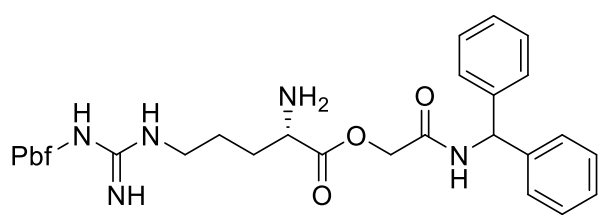

2-(benzhydrylamino)-2-oxoethyl

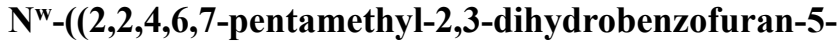
yl)sulfonyl)- $L$-argininate

$\mathbf{H}-\mathbf{A r g}(\mathbf{P b f})-\mathbf{O B c M}$ was prepared as a white powder $(357.7 \mathrm{mg})$ in $55 \%$ yield.

${ }^{1} \mathrm{H}$ NMR $\left(500 \mathrm{MHz}\right.$, DMSO- $\left.d_{6}\right) \delta 9.08(\mathrm{~d}, J=8.5 \mathrm{~Hz}, 1 \mathrm{H}), 8.42(\mathrm{~s}, 2 \mathrm{H}), 7.36-7.22(\mathrm{~m}, 10 \mathrm{H}), 6.13(\mathrm{~d}$, $J=8.5 \mathrm{~Hz}, 1 \mathrm{H}), 4.79(\mathrm{q}, J=14.6 \mathrm{~Hz}, 2 \mathrm{H}), 4.14(\mathrm{~d}, J=5.1 \mathrm{~Hz}, 1 \mathrm{H}), 3.05(\mathrm{dd}, J=12.4,6.5 \mathrm{~Hz}, 2 \mathrm{H}), 2.95$ (s, 2H), 2.48 (s, 3H), 2.42 (s, 3H), $2.00(\mathrm{~s}, 3 \mathrm{H}), 1.90-1.70(\mathrm{~m}, 2 \mathrm{H}), 1.66-1.51(\mathrm{~m}, 2 \mathrm{H}), 1.40(\mathrm{~s}, 6 \mathrm{H})$. ${ }^{13} \mathrm{C}$ NMR $\left(126 \mathrm{MHz}, \mathrm{DMSO}-d_{6}\right) \delta 169.2,165.1,158.3,158.0,157.7,157.4,156.1,141.9,137.2$, 131.4, 128.4, 127.2, 127.2, 127.1, 124.2, 117.1, 116.2, 86.3, 63.2, 55.9, 51.5, 42.4, 28.2, 27.5, 18.9, 17.6, 12.2 .

ESI-MS, m/z: $[\mathrm{M}+\mathrm{H}]^{+}$calcd for $\mathrm{C}_{34} \mathrm{H}_{44} \mathrm{~N}_{5} \mathrm{O}_{6} \mathrm{~S}^{+}$650.3012; found 650.3054 .<smiles>NC(Cc1cn(C(=O)c2ccccc2)c2ccccc12)C(=O)OCC(=O)NC(c1ccccc1)c1ccccc1</smiles> 
tert-butyl (S)-3-(2-amino-3-(2-(benzhydrylamino)-2-oxoethoxy)-3-oxopropyl)-1H-indole-1carboxylate

H-Trp(Boc)-OBcM was prepared as a white powder (179.4 mg) in 34\% yield.

${ }^{1} \mathrm{H}$ NMR (500 MHz, DMSO- $\left.d_{6}\right) \delta 9.10(\mathrm{~d}, J=8.5 \mathrm{~Hz}, 1 \mathrm{H}), 8.48(\mathrm{~s}, 2 \mathrm{H}), 8.06(\mathrm{~d}, J=8.3 \mathrm{~Hz}, 1 \mathrm{H})$, $7.71(\mathrm{~s}, 1 \mathrm{H}), 7.61(\mathrm{~d}, J=7.8 \mathrm{~Hz}, 1 \mathrm{H}), 7.40-7.22(\mathrm{~m}, 12 \mathrm{H}), 6.16(\mathrm{~d}, J=8.5 \mathrm{~Hz}, 1 \mathrm{H}), 4.80(\mathrm{q}, J=$ $14.6 \mathrm{~Hz}, 2 \mathrm{H}), 4.46$ (s, 1H), 3.29-3.27 (m, 2H), 1.61 (s, 9H). ${ }^{13} \mathrm{C}$ NMR (126 MHz, DMSO-d 6 ) $\delta 168.7$, 165.2, 158.1, 157.8, 148.9, 141.9, 134.8, 129.6, 128.4, 128.4, 127.2, 127.2, 127.2, 125.1, 124.3, $122.0,118.1,114.8,112.9,83.2,63.3,55.9,51.6,27.6,25.4$.

ESI-MS, m/z: [M+H] $]^{+}$calcd for $\mathrm{C}_{31} \mathrm{H}_{34} \mathrm{~N}_{3} \mathrm{O}_{5}+528.2498$; found 528.2470.<smiles>CCC(C)Oc1ccc(C[C@H](N)C(=O)OCC(=O)NC(c2ccccc2)c2ccccc2)cc1</smiles>

2-(benzhydrylamino)-2-oxoethyl (S)-2-amino-3-(4-(tert-butoxy)phenyl)propanoate

H-Tyr( $\left.{ }^{(} \mathbf{B u}\right)-O B c M$ was prepared as a white powder $(237.2 \mathrm{mg})$ in $57 \%$ yield.

${ }^{1} \mathrm{H}$ NMR $\left(500 \mathrm{MHz}, \mathrm{DMSO}-d_{6}\right) \delta 9.08(\mathrm{~d}, J=8.5 \mathrm{~Hz}, 1 \mathrm{H}), 8.45(\mathrm{~s}, 2 \mathrm{H}), 7.38-7.25(\mathrm{~m}, 10 \mathrm{H}), 7.21$ $-7.14(\mathrm{~m}, 2 \mathrm{H}), 6.92-6.85(\mathrm{~m}, 2 \mathrm{H}), 6.14(\mathrm{~d}, J=8.5 \mathrm{~Hz}, 1 \mathrm{H}), 4.77(\mathrm{~s}, 2 \mathrm{H}), 4.38(\mathrm{~d}, J=6.0 \mathrm{~Hz}, 1 \mathrm{H})$, 3.10-3.07 (m, 2H), 1.28 (s, 9H). ${ }^{13} \mathrm{C}$ NMR (126 MHz, DMSO- $\left.d_{6}\right) \delta 168.8,165.1,154.3,141.9,130.1$, $128.8,128.4,127.2,127.1,123.6,77.8,63.2,55.9,53.0,35.2,28.5$.

ESI-MS, m/z: [M+H] $]^{+}$calcd for $\mathrm{C}_{28} \mathrm{H}_{33} \mathrm{~N}_{2} \mathrm{O}_{4}{ }^{+} 416.2440$; found 416.2462.<smiles>O=C(COC(=O)[C@@H]1CCCN1)NC(c1ccccc1)c1ccccc1</smiles>

\section{2-(benzhydrylamino)-2-oxoethyl $L$-prolinate}

H-Pro-OBcM was prepared as a white powder $(162.9 \mathrm{mg})$ in $48 \%$ yield.

${ }^{1} \mathrm{H}$ NMR (500 MHz, DMSO- $\left.d_{6}\right) \delta 9.30$ (s, 1H), 9.08 (d, $J=8.4$ Hz, 1H), 7.29-7.24 (m, 10H), 6.12 $(\mathrm{d}, J=8.4 \mathrm{~Hz}, 1 \mathrm{H}), 4.85(\mathrm{~d}, J=14.6 \mathrm{~Hz}, 1 \mathrm{H}), 4.77(\mathrm{~d}, J=14.6 \mathrm{~Hz}, 1 \mathrm{H}), 4.51(\mathrm{dd}, J=8.6,7.2 \mathrm{~Hz}$, 1H), $3.28-3.17(\mathrm{~m}, 2 \mathrm{H}), 2.30-2.28(\mathrm{~m}, 1 \mathrm{H}), 2.16-2.06(\mathrm{~m}, 1 \mathrm{H}), 1.99-1.84(\mathrm{~m}, 2 \mathrm{H}) .{ }^{13} \mathrm{C}$ NMR (126 MHz, DMSO-d $d_{6} \delta 168.7,165.1,141.9,141.9,128.4,127.2,127.1,63.3,58.4,55.9,45.5,27.8$, 22.8 .

ESI-MS, m/z: $[\mathrm{M}+\mathrm{H}]^{+}$calcd for $\mathrm{C}_{20} \mathrm{H}_{23} \mathrm{~N}_{2} \mathrm{O}_{3}{ }^{+}$339.1709; found 339.1713.

\subsection{Synthesis of linker (compounds 5a-5d)}

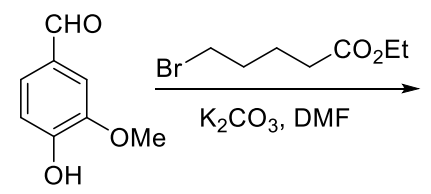<smiles>CCOC(=O)CCCCOc1ccc(C=O)cc1OC</smiles>
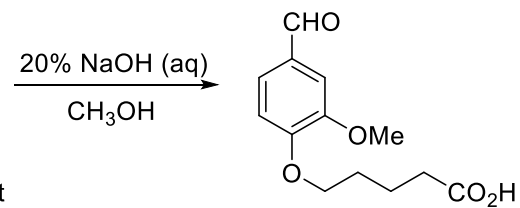

Scheme 3. Synthesis of linker 1(compounds 5a).

Synthesis procedure: $\mathrm{K}_{2} \mathrm{CO}_{3}(20 \mathrm{mmol})$ was added to 4-hydroxy-3-methoxybenzaldehyde (5 $\mathrm{mmol}$ ) in DMF and stirred at $\mathrm{rt}$ for $30 \mathrm{~min}$. Ethyl 5-chloropentanoate 10 (mmol) was added to the 
mixture and heated at $50^{\circ} \mathrm{C}$ for $30 \mathrm{~min}$. After completion of the reaction, the mixture was cooled to rt, diluted with $\mathrm{H}_{2} \mathrm{O}(150 \mathrm{~mL})$, and extracted with ethyl acetate $(150 \mathrm{~mL} \times 2)$. The organic phases were combined, hydrolyzed with Sodium hydroxide aqueous solution for $2 \mathrm{~h}$, then adjust $\mathrm{pH}$ to neutral by aqueous hydrochloric acid. Then washed with $\mathrm{H}_{2} \mathrm{O}(200 \mathrm{~mL} \times 2)$ and brine $(200 \mathrm{~mL} \times 2)$, dried over anhydrous $\mathrm{Na}_{2} \mathrm{SO}_{4}$, evaporated under vacuum, and purified by flash column chromatography (PE/EA 1:1). Linker 1 (5a) was prepared as a white powder $(224.3 \mathrm{mg})$ in $89 \%$ yield.<smiles>COc1cc(C=O)ccc1OCCCCC(=O)O</smiles>

\section{5-(4-formyl-2-methoxyphenoxy)pentanoic acid}

${ }^{1} \mathrm{H} \mathrm{NMR}\left(500 \mathrm{MHz}, \mathrm{CDCl}_{3}\right) \delta 9.85(\mathrm{~s}, 1 \mathrm{H}), 7.44(\mathrm{dd}, J=8.2,1.8 \mathrm{~Hz}, 1 \mathrm{H}), 7.41(\mathrm{~d}, J=1.8 \mathrm{~Hz}, 1 \mathrm{H})$, $6.96(\mathrm{~d}, J=8.2 \mathrm{~Hz}, 1 \mathrm{H}), 4.13(\mathrm{t}, J=6.3 \mathrm{~Hz}, 2 \mathrm{H}), 3.93(\mathrm{~s}, 3 \mathrm{H}), 2.48(\mathrm{t}, J=7.3 \mathrm{~Hz}, 2 \mathrm{H}), 2.01-1.92$ $(\mathrm{m}, 2 \mathrm{H}), 1.91-1.81(\mathrm{~m}, 2 \mathrm{H}) .{ }^{13} \mathrm{C}$ NMR $\left(126 \mathrm{MHz}, \mathrm{CDCl}_{3}\right) \delta 191.0,178.5,153.9,149.8,130.0$, $126.8,111.3,109.2,68.5,56.0,33.4,28.2,21.3$.

ESI-MS, m/z: [M+H]+ calcd for $\mathrm{C}_{13} \mathrm{H}_{17} \mathrm{O}_{5}{ }^{+} 253.1078$, ; found 253.1077 .<smiles>COc1cc(OCCCCC(=O)O)ccc1C=O</smiles>

The synthesis steps of linker $2 \mathbf{( 5 b )}$ are the same as above, linker $\mathbf{2}(\mathbf{5 b})$ was prepared as a yellow powder (205.9 $\mathrm{mg})$ in $82 \%$ yield.

\section{5-(4-formyl-3-methoxyphenoxy)pentanoic acid}

${ }^{1} \mathrm{H}$ NMR $\left(500 \mathrm{MHz}, \mathrm{CDCl}_{3}\right) \delta 10.28(\mathrm{~s}, 1 \mathrm{H}), 7.80(\mathrm{~d}, J=8.7 \mathrm{~Hz}, 1 \mathrm{H}), 6.53(\mathrm{dd}, J=8.7,2.0 \mathrm{~Hz}$, $1 \mathrm{H}), 6.44(\mathrm{~d}, J=2.0 \mathrm{~Hz}, 1 \mathrm{H}), 4.06(\mathrm{t}, J=5.7 \mathrm{~Hz}, 2 \mathrm{H}), 3.90(\mathrm{~s}, 3 \mathrm{H}), 2.46(\mathrm{t}, J=6.8 \mathrm{~Hz}, 2 \mathrm{H}), 1.90-$ $1.83(\mathrm{~m}, 4 \mathrm{H}) .{ }^{13} \mathrm{C} \mathrm{NMR}\left(126 \mathrm{MHz}, \mathrm{CDCl}_{3}\right) \delta 188.4,177.4,165.5,163.6,130.8,118.9,106.0,98.3$, 67.7, 55.6, 33.2, 28.3, 21.3, 21.3.

ESI-MS, m/z: $[\mathrm{M}+\mathrm{H}]+$ calcd for $\mathrm{C}_{13} \mathrm{H}_{17} \mathrm{O}_{5}{ }^{+} 253.1078$; found 253.1075 .<smiles>COc1cc(C=O)cc(OC)c1OCCCCC(=O)O</smiles>

The synthesis steps of linker $3(\mathbf{5 c})$ are the same as above, Linker $3(\mathbf{5 c})$ was prepared as a white powder $(219.3 \mathrm{mg})$ in $87 \%$ yield.

\section{5-(4-formyl-2,6-dimethoxyphenoxy)pentanoic acid}


${ }^{1} \mathrm{H}$ NMR $\left(500 \mathrm{MHz}, \mathrm{CDCl}_{3}\right) \delta 9.87(\mathrm{~s}, 1 \mathrm{H}), 7.13(\mathrm{~s}, 2 \mathrm{H}), 4.09(\mathrm{t}, J=5.7 \mathrm{~Hz}, 2 \mathrm{H}), 3.92(\mathrm{~s}, 6 \mathrm{H}), 2.50$

$-2.43(\mathrm{~m}, 2 \mathrm{H}), 1.93-1.77(\mathrm{~m}, 4 \mathrm{H}) .{ }^{13} \mathrm{C} \mathrm{NMR}\left(126 \mathrm{MHz}, \mathrm{CDCl}_{3}\right) \delta 191.2,179.3,153.8,142.7$, 131.7, 106.7, 72.3, 56.5, 33.4, 29.3, 21.2.

ESI-MS, m/z: $[\mathrm{M}+\mathrm{H}]+$ calcd for $\mathrm{C}_{13} \mathrm{H}_{17} \mathrm{O}_{5}{ }^{+} 283.1178$; found 283.1174 .<smiles>COc1ccc(C=O)c(OCCCCC(=O)O)c1</smiles>

The synthesis steps of linker 4 (5d) are the same as above, linker $3(\mathbf{5 d})$ was prepared as a white powder $(221.6 \mathrm{mg})$ in $88 \%$ yield.

\section{5-(2-formyl-5-methoxyphenoxy)pentanoic acid}

${ }^{1} \mathrm{H}$ NMR $\left(500 \mathrm{MHz}, \mathrm{CDCl}_{3}\right) \delta 10.31(\mathrm{~s}, 1 \mathrm{H}), 7.81(\mathrm{~d}, J=8.7 \mathrm{~Hz}, 1 \mathrm{H}), 6.57-6.51(\mathrm{~m}, 1 \mathrm{H}), 6.42(\mathrm{~d}$, $J=2.2 \mathrm{~Hz}, 1 \mathrm{H}), 4.10-4.04(\mathrm{~m}, 2 \mathrm{H}), 3.86(\mathrm{~s}, 3 \mathrm{H}), 2.47(\mathrm{t}, J=7.1 \mathrm{~Hz}, 2 \mathrm{H}), 1.97-1.81(\mathrm{~m}, 4 \mathrm{H}) .{ }^{13} \mathrm{C}$ NMR $\left(126 \mathrm{MHz}, \mathrm{CDCl}_{3}\right) \delta 188.4,178.4,166.2,163.0,130.5,119.0,105.9,98.5,67.89,55.6,33.3$, 28.3, 21.3.

ESI-MS, m/z: $[\mathrm{M}+\mathrm{H}]+$ calcd for $\mathrm{C}_{13} \mathrm{H}_{17} \mathrm{O}_{5}{ }^{+} 253.1078$; found 253.1069 .

\section{General Methods for Peptide Synthesis}

\section{General Methods for Peptide Synthesis by Acyl Azid-ISPPS}

AM resin $(250 \mathrm{mg}, 1.0 \mathrm{mmol} / \mathrm{g})$ was swelled in DMF/DCM (1:1) for $30 \mathrm{~min}$, then was added into a $10 \mathrm{~mL}$ peptide synthesis tube. After removing the solvent, for linker-4: a solution of the linker-4 (252 mg, $0.5 \mathrm{mmol}, 2.0$ equiv) and 0.4 M HATU ((2.5 mL, 4 equiv) and $0.8 \mathrm{M}$ DIPEA (2.5 mL, 8 equiv) in DMF was added and the reaction mixture was shaken for $1.5 \mathrm{~h}$ at room temperature. For linker PA: a solution of the 1,5-pentanedioic acid ( $57 \mathrm{mg}, 0.5 \mathrm{mmol}, 2.0$ equiv) and pyridine (40.1 $\mu \mathrm{L}, 0.5 \mathrm{mmol}, 2.0$ equiv) in DMF was added and the reaction mixture was shaken for $1.5 \mathrm{~h}$ at room temperature.

For linker-4 using RA reaction of amino acids: The loading resin was treated with a solution of AA-OBn (1.0 mmol, 4 equiv), $\mathrm{NaBH}_{3} \mathrm{CN}$ (2.0 mmol, 8 equiv) and $\mathrm{AcOH}(0.25 \mathrm{mmol}, 0.1$ equiv) in $5 \mathrm{ml}$ DMF for $2.0 \mathrm{hr}$, then washed by DMF and DCM for three times.

For linker PA using coupling of amino acids: The loading resin was treated with a solution of $\mathrm{H}$ $\mathrm{X}_{\mathrm{AA}-\mathrm{OBn}}$ ( $1 \mathrm{mmol}, 4$ equiv), HATU ( $1 \mathrm{mmol}, 4$ equiv), and DIPEA ( 2 mmol, 8 equiv) in $5 \mathrm{~mL} \mathrm{DMF}$ for $1.5 \mathrm{~h}$, then washed by DMF and DCM for three times.

For hydrazinolysis: The resin was treated with $20 \% \mathrm{NH}_{2} \mathrm{NH}_{2}$ in DMF $(5 \mathrm{~mL})$, then washed by DMF and DCM for three times. (Note: The reaction time is $1-2 \mathrm{~h}$ with benzyl ester, 15 min with (benzhydryl-carbamoyl)-methyl ester.)

For acyl azide coupling method: The resin was swelled in $5.0 \mathrm{~mL} \mathrm{DMF}$ and treated with $5 \mathrm{M}$ sodium nitrite ( $500 \mu \mathrm{L}, 2.5 \mathrm{mmol}, 10$ equiv) and adjust $\mathrm{pH} \sim 3$ for 15-30 min. Then a solution of amino acid ester ( $1.25 \mathrm{mmol}, 5.0$ equiv) was added in resin system and adjust $\mathrm{pH} \sim 8$ for $30-45 \mathrm{~min}$, then washed by DMF and DCM for three times.

Repeating the hydrazinolysis and acyl azide coupling process, peptide chain can be elongated successively. 
The cleavage cocktail of TFA/water/TIPS (95/2.5/2.5) was employed and the cleavage was carried out for $1.5 \mathrm{~h}$. Then, the resin was removed by filtration and the TFA in the filtrate was removed by blowing $\mathrm{N}_{2}$. The crude peptides were obtained by precipitating with cold diethyl ether.

\section{General Methods for Peptide Synthesis by Fmoc-SPPS}

Rink amide AM resin (416.7 mg, $0.6 \mathrm{mmol} / \mathrm{g}$ ) was added into a $10 \mathrm{~mL}$ peptide synthesis tube following being swelled in DMF/DCM (1:1) for $30 \mathrm{~min}$. After removing the solvent, a solution of the Fmoc-AA-OBn (445 mg, $1 \mathrm{mmol}, 4.0$ equiv) and $0.4 \mathrm{M} \mathrm{HATU} \mathrm{((2.5} \mathrm{mL,} 4$ equiv) and $0.8 \mathrm{M}$ DIPEA ( $2.5 \mathrm{~mL}, 8$ equiv) in DMF was added and the reaction mixture was shaken for 1.5 hour at room temperature. Then the solvent was removed and the system was washed with DMF $(20 \mathrm{~mL})$ and DCM $(20 \mathrm{~mL})$, followed by treating with a solution of $20 \%$ piperidine/DMF for $10 \mathrm{~min}$ twice. Afterwards the resin was washed with DMF (10 mL), DCM (3 x $10 \mathrm{~mL})$.

Repeating the coupling and deprotection steps, peptide chain can be elongated successively. After the completion of peptide elongation, the resin was capped with acetic anhydride and pyridine.

\section{Hydrazinolysis result: Ac-FFGN(AA)-OBn / Ac-FFGN(AA)-NHNH}

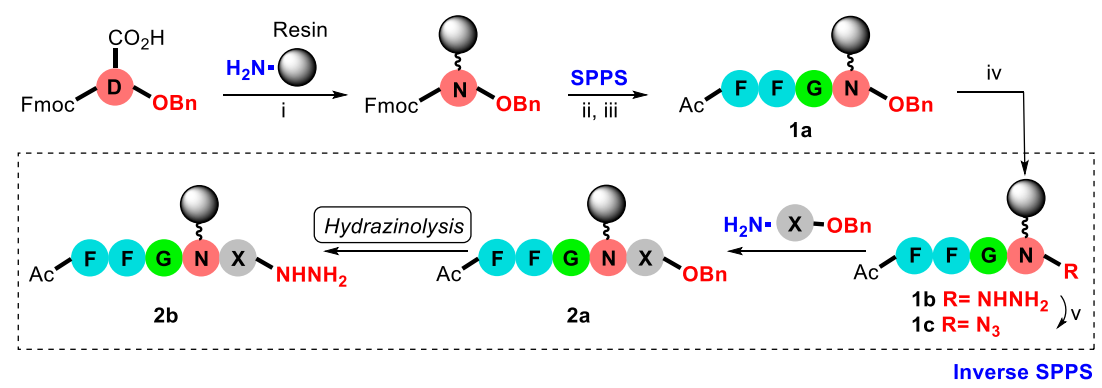

i) HATU, DIPEA; ii) Fmoc SPPS cycle: 1) piperdine, DMF; 2) HATU, DIPEA, DMF; iii) acetic anhydride, pyridine iv) $20 \% \mathrm{NH}_{2} \mathrm{NH}_{2}$ in DMF; v) $\mathrm{NaNO}_{2}$ (Sodium nitrite solution), $\mathrm{HCl}$

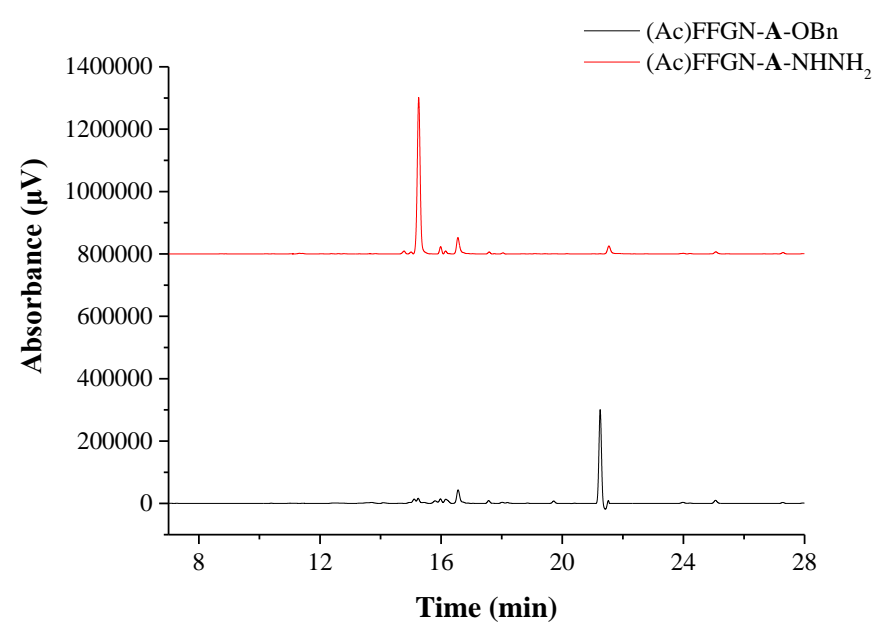



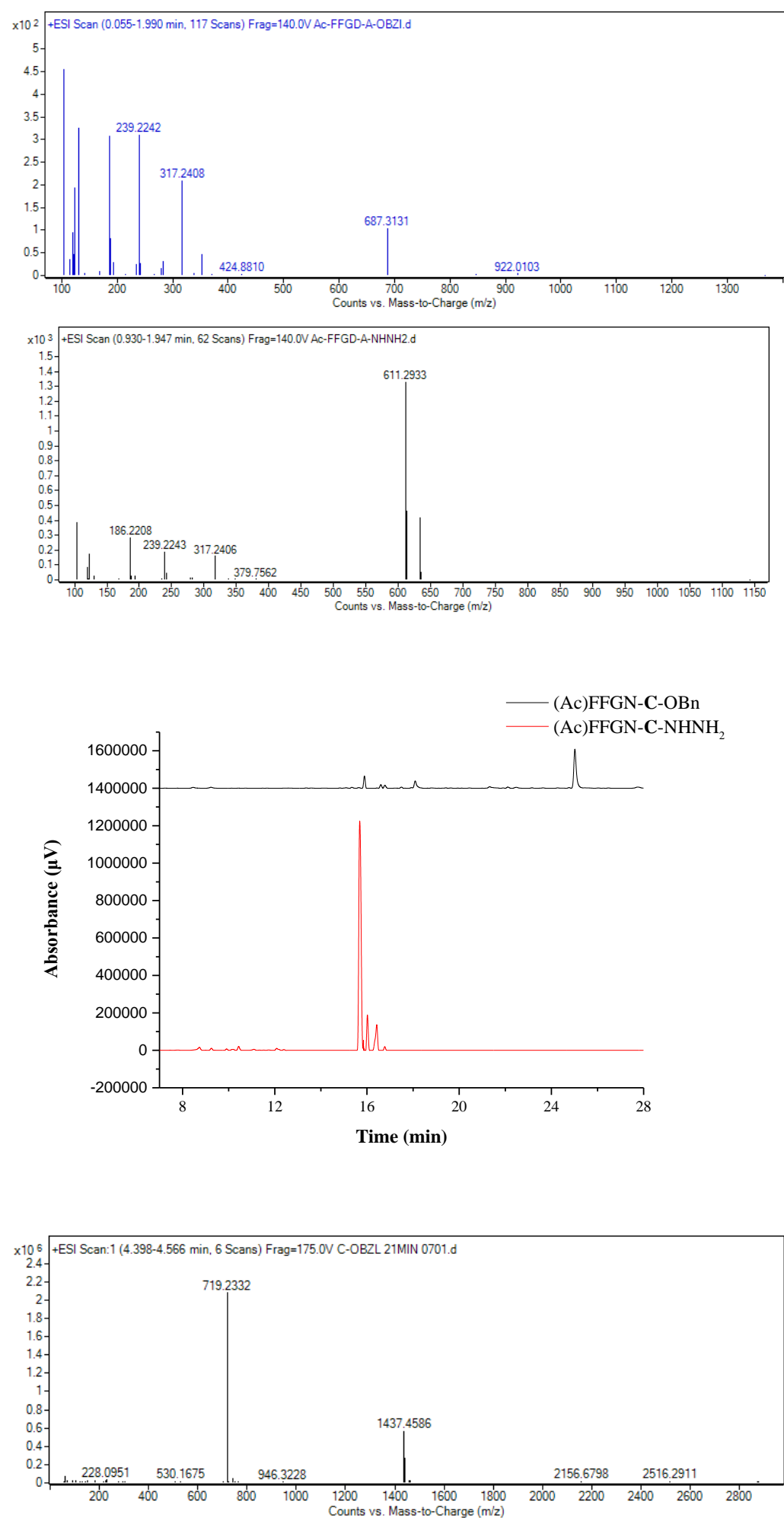

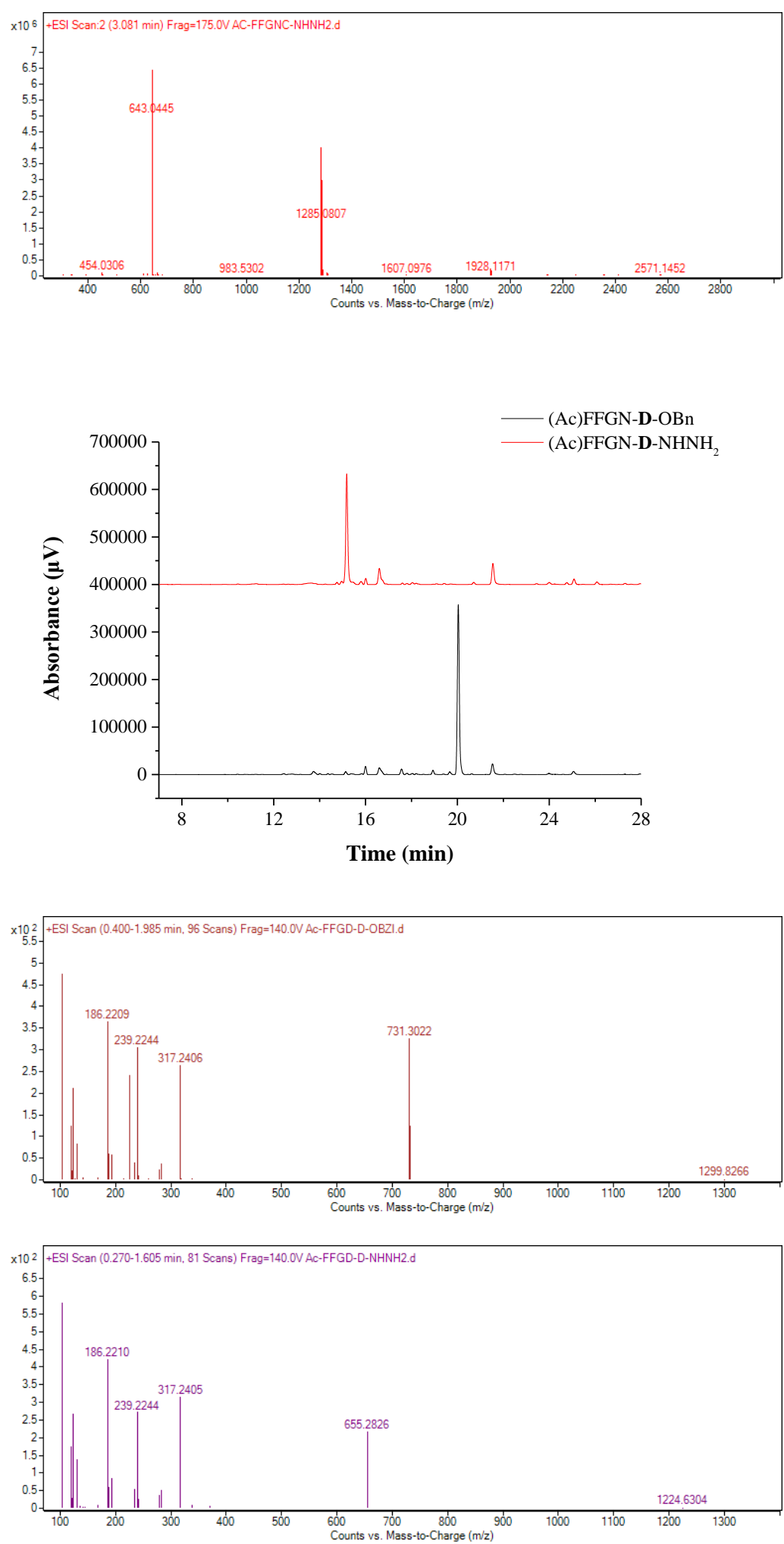

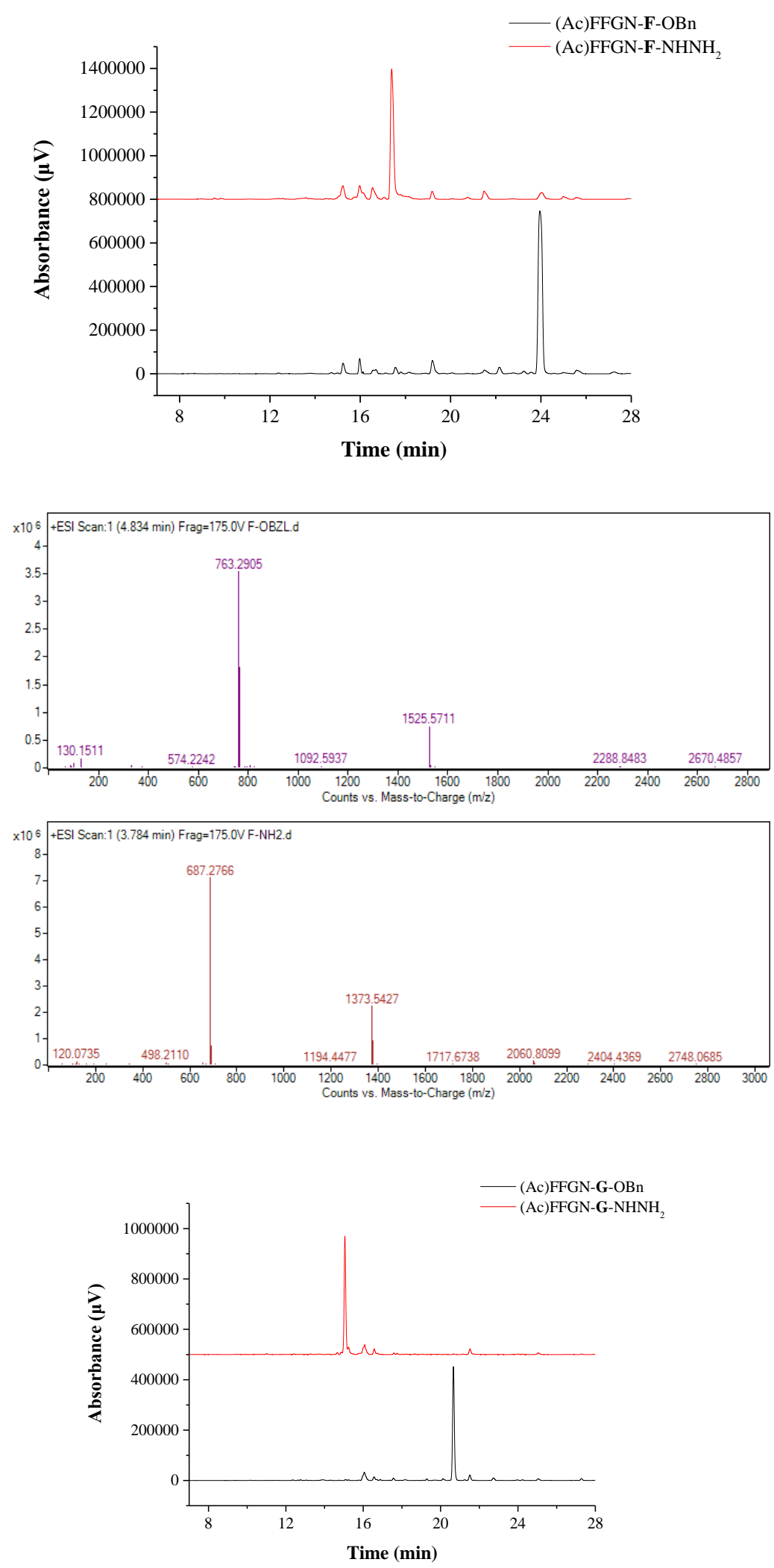

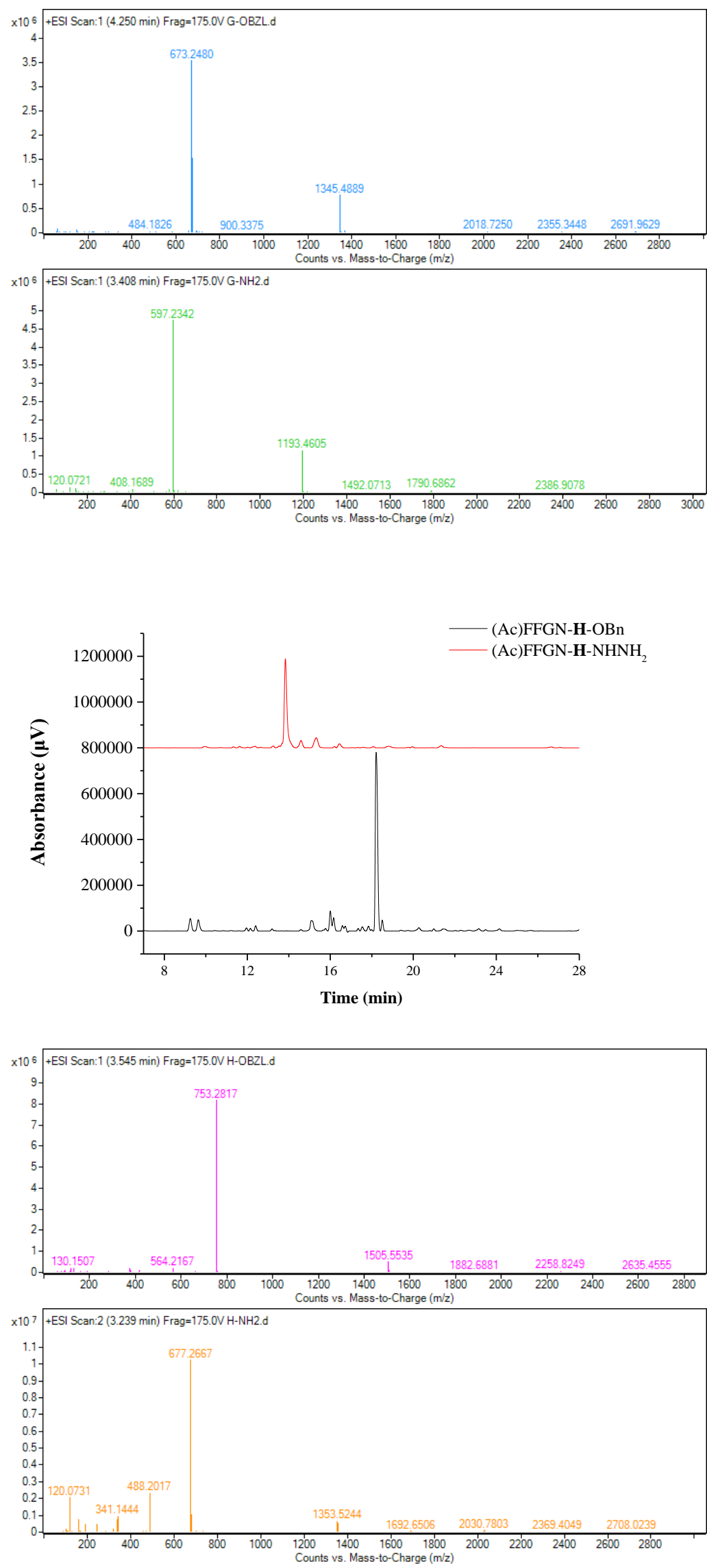

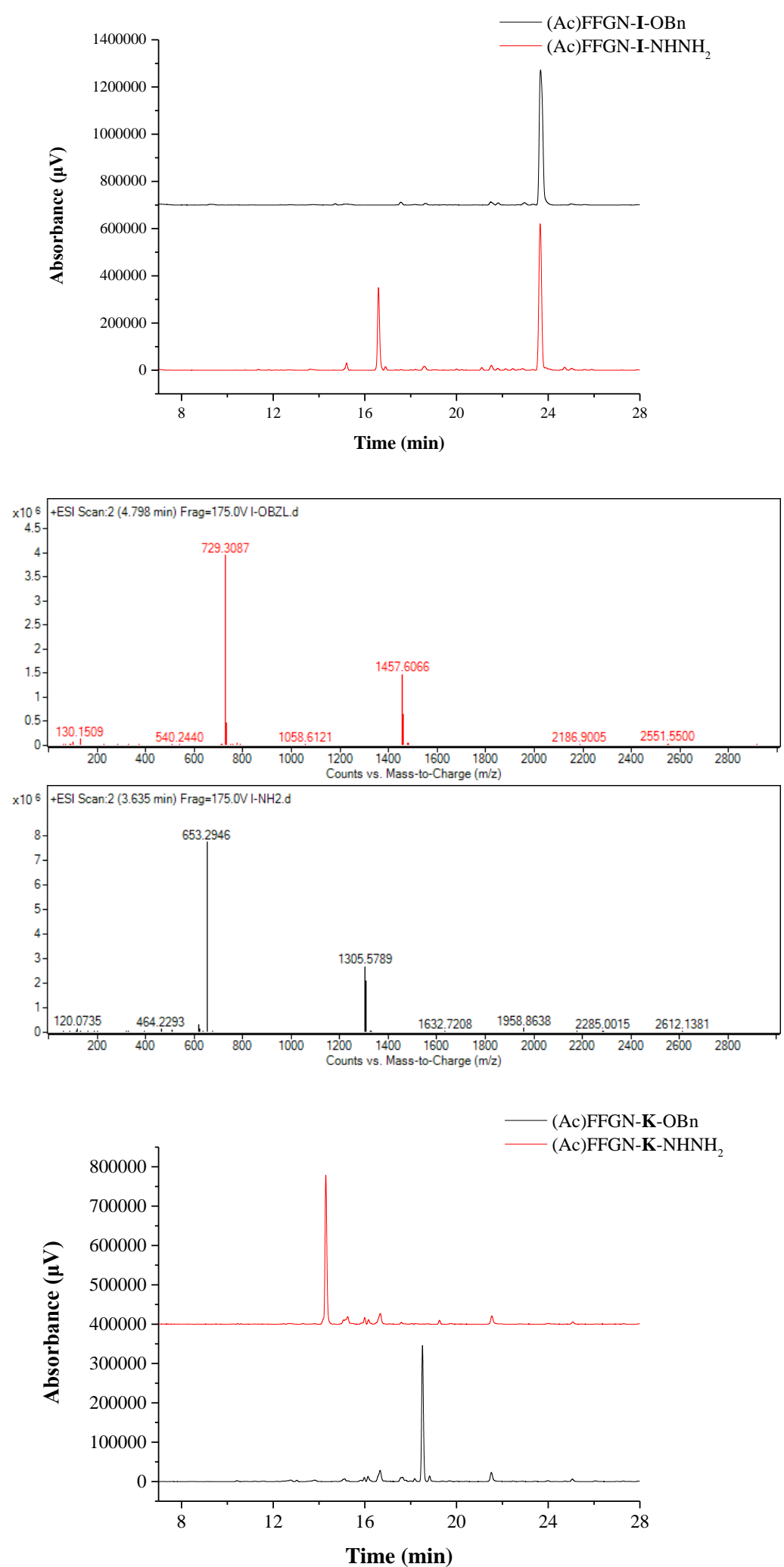

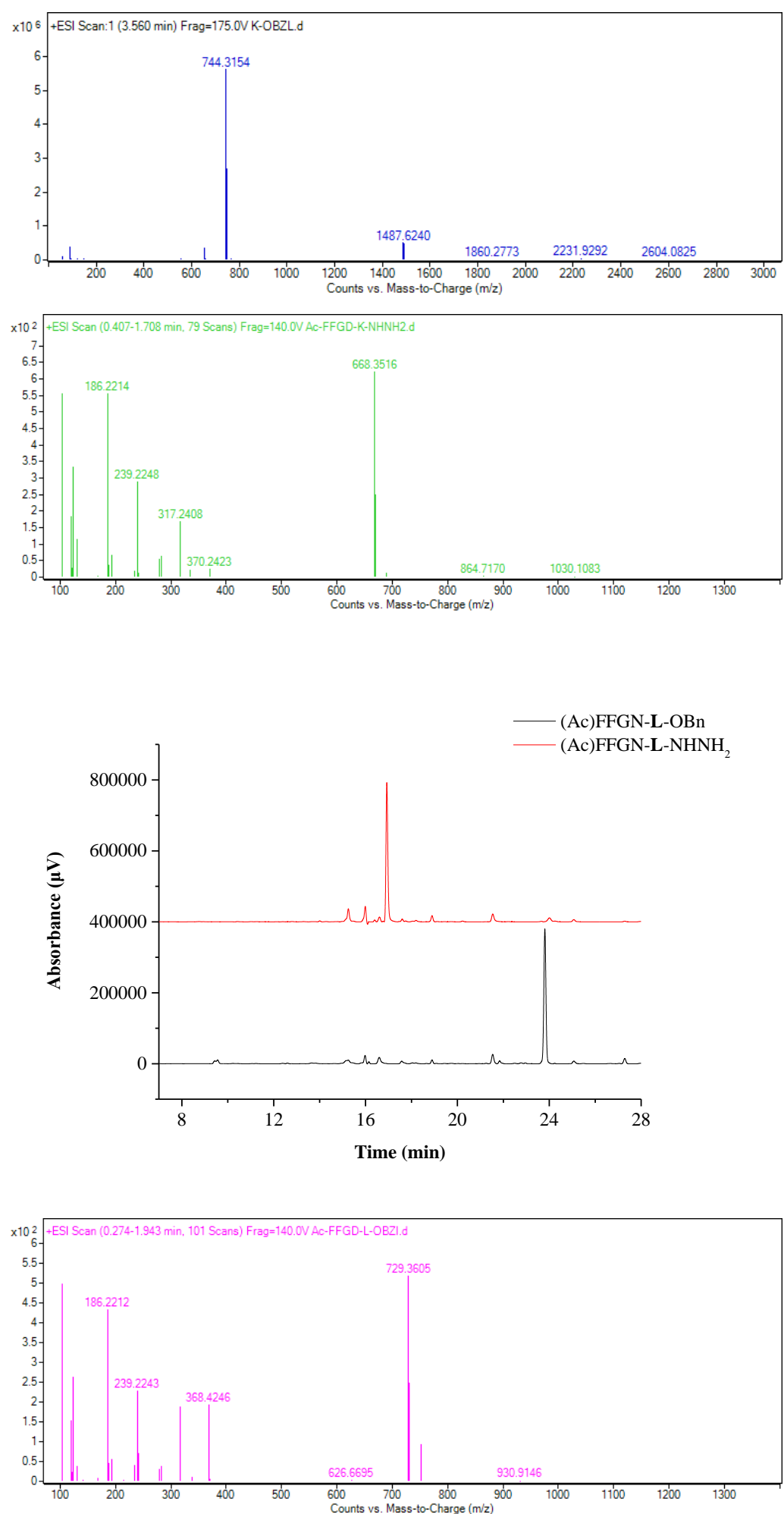

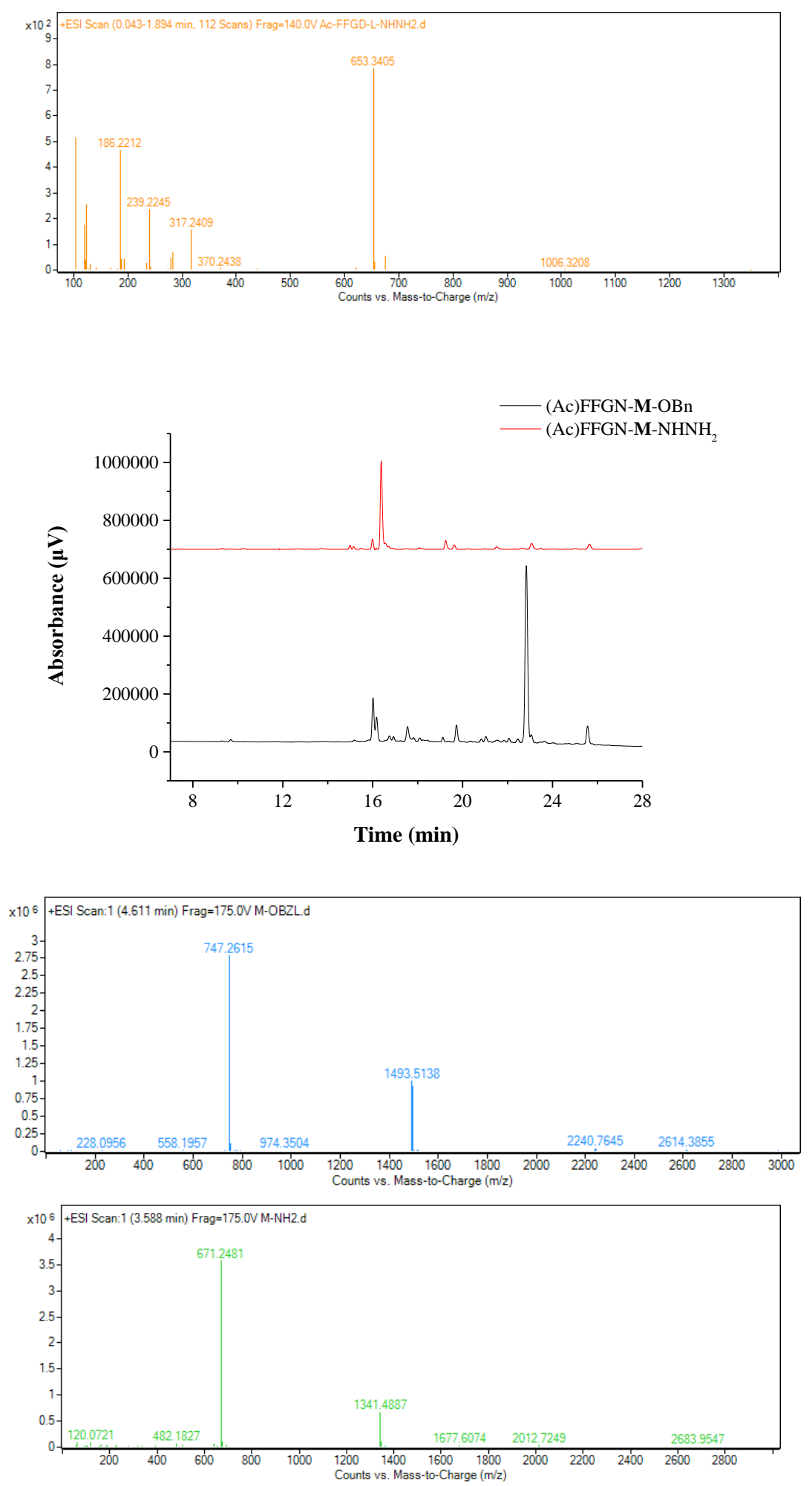

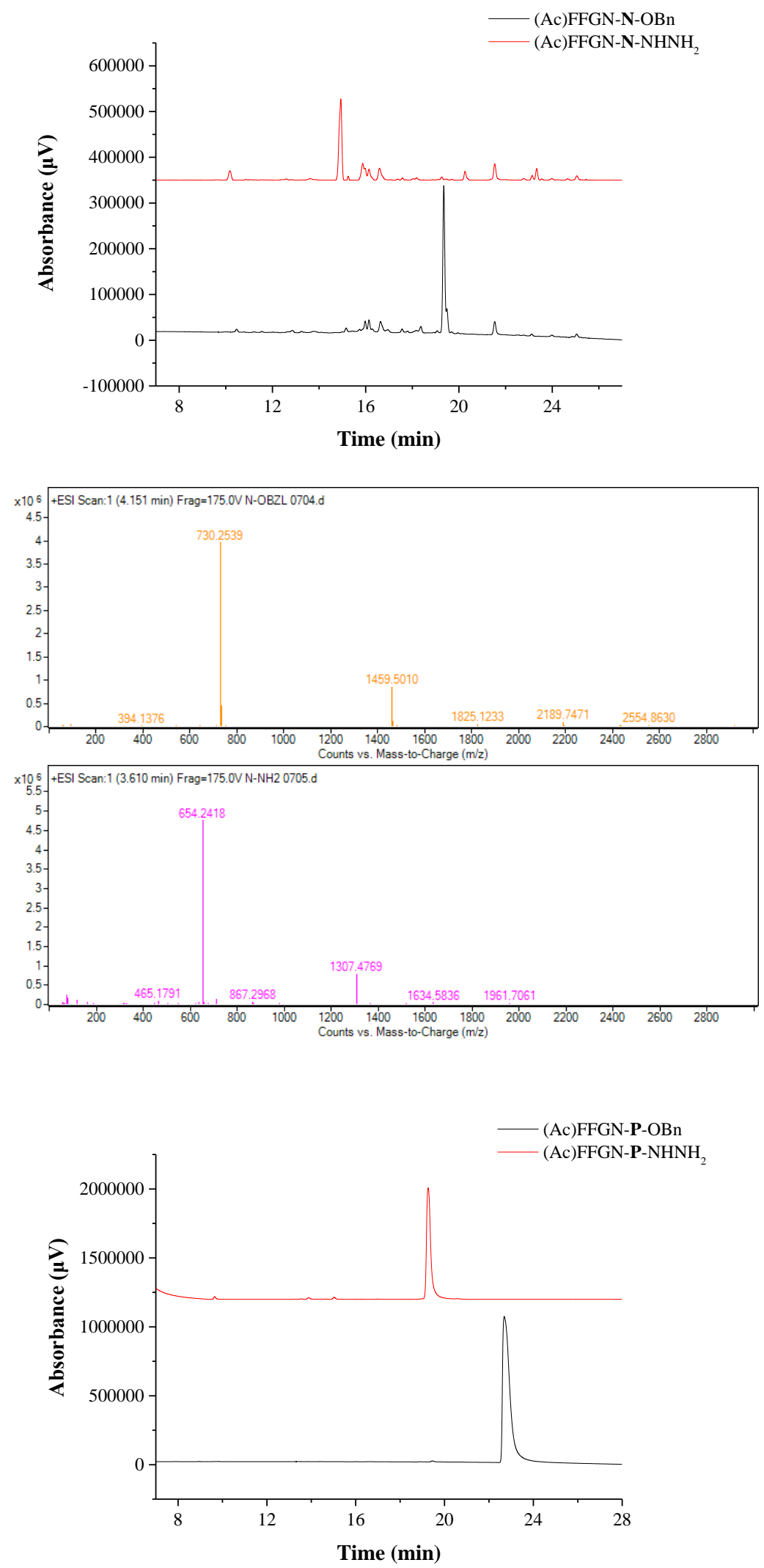

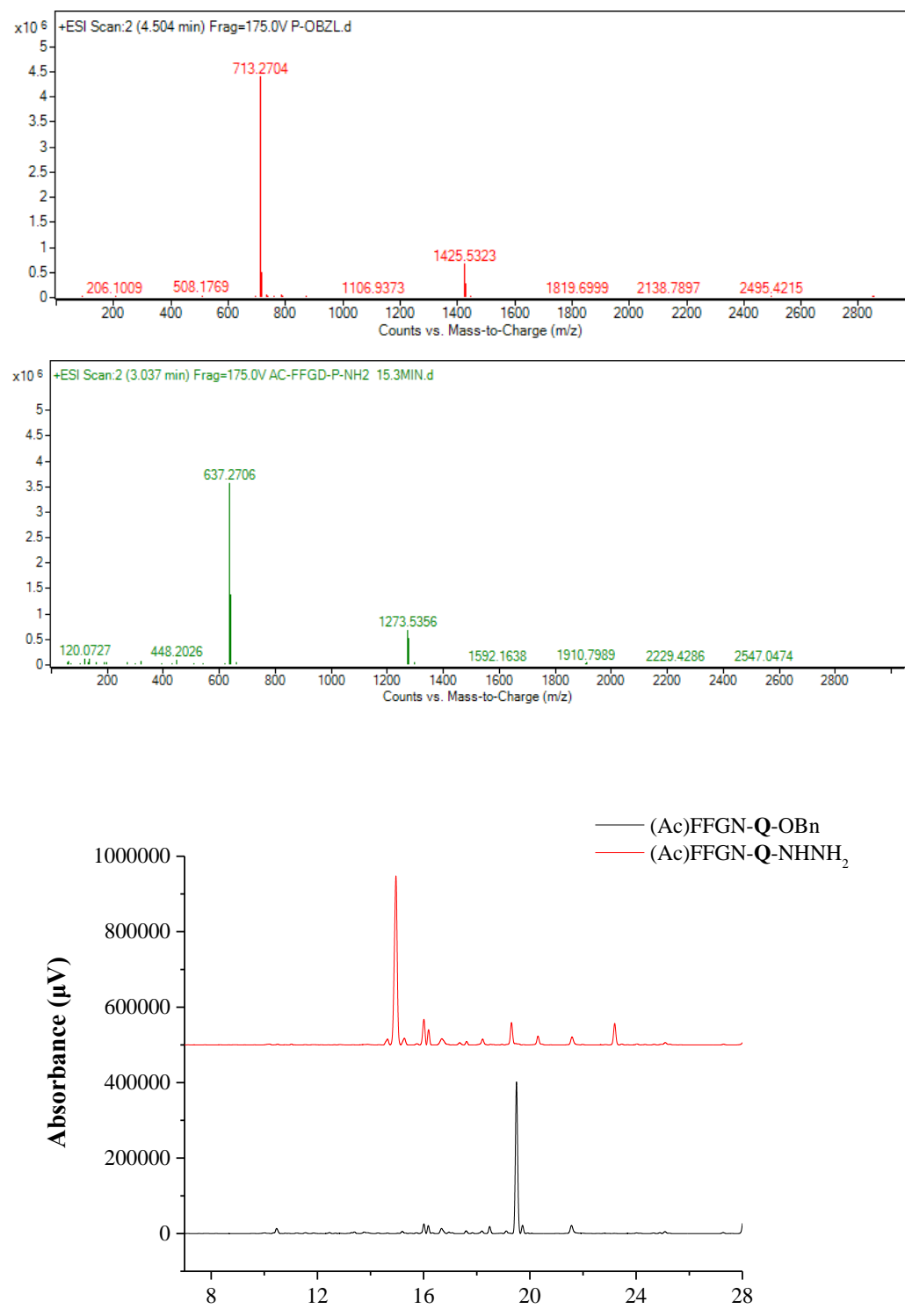

Time (min)
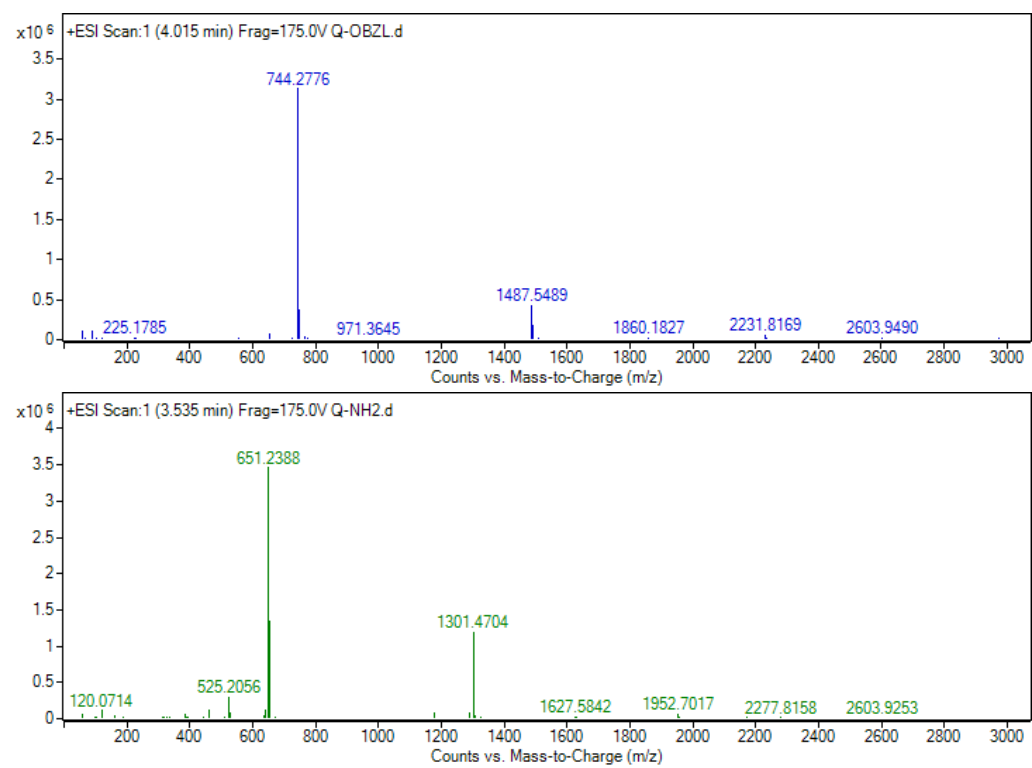

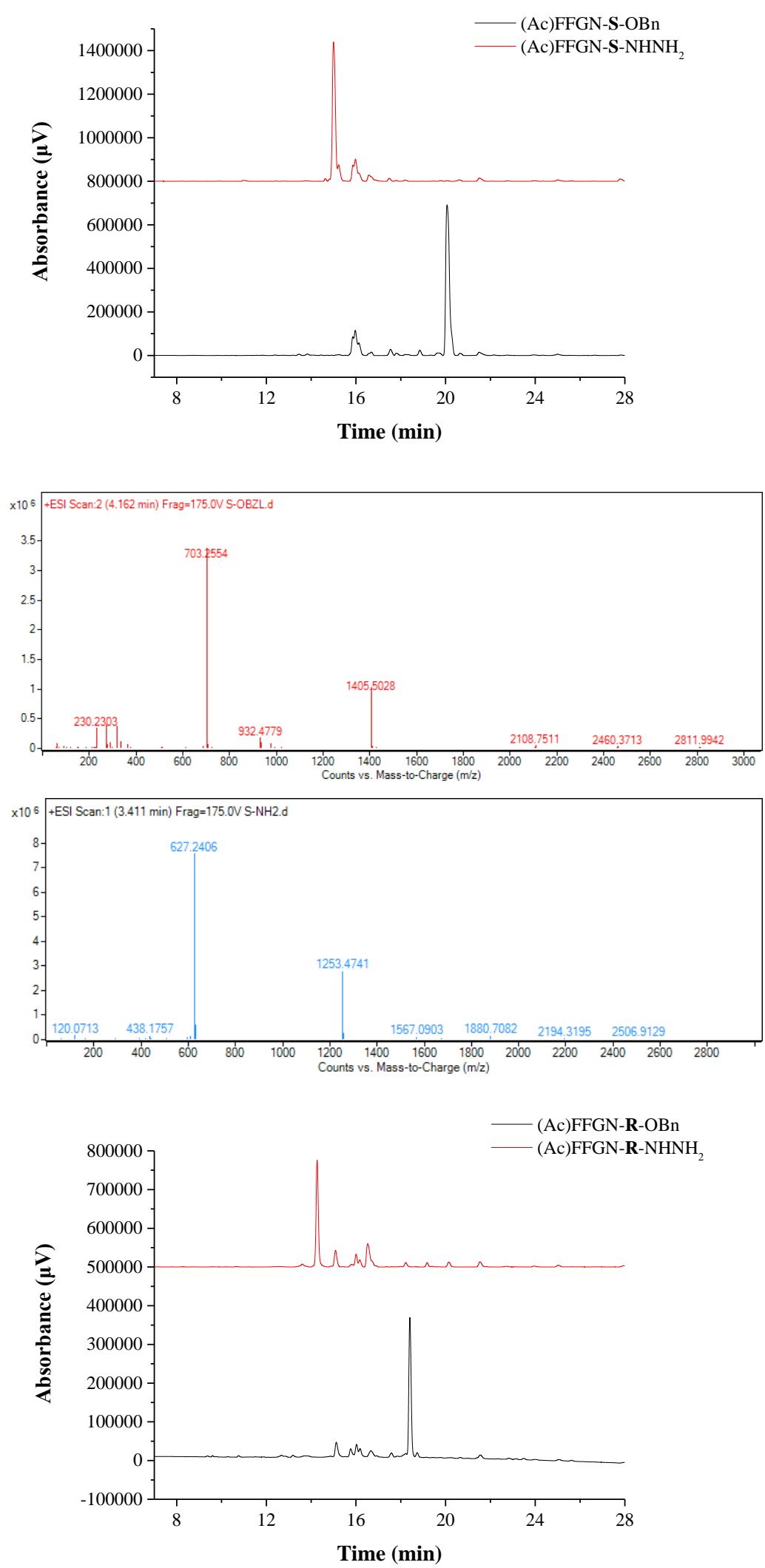

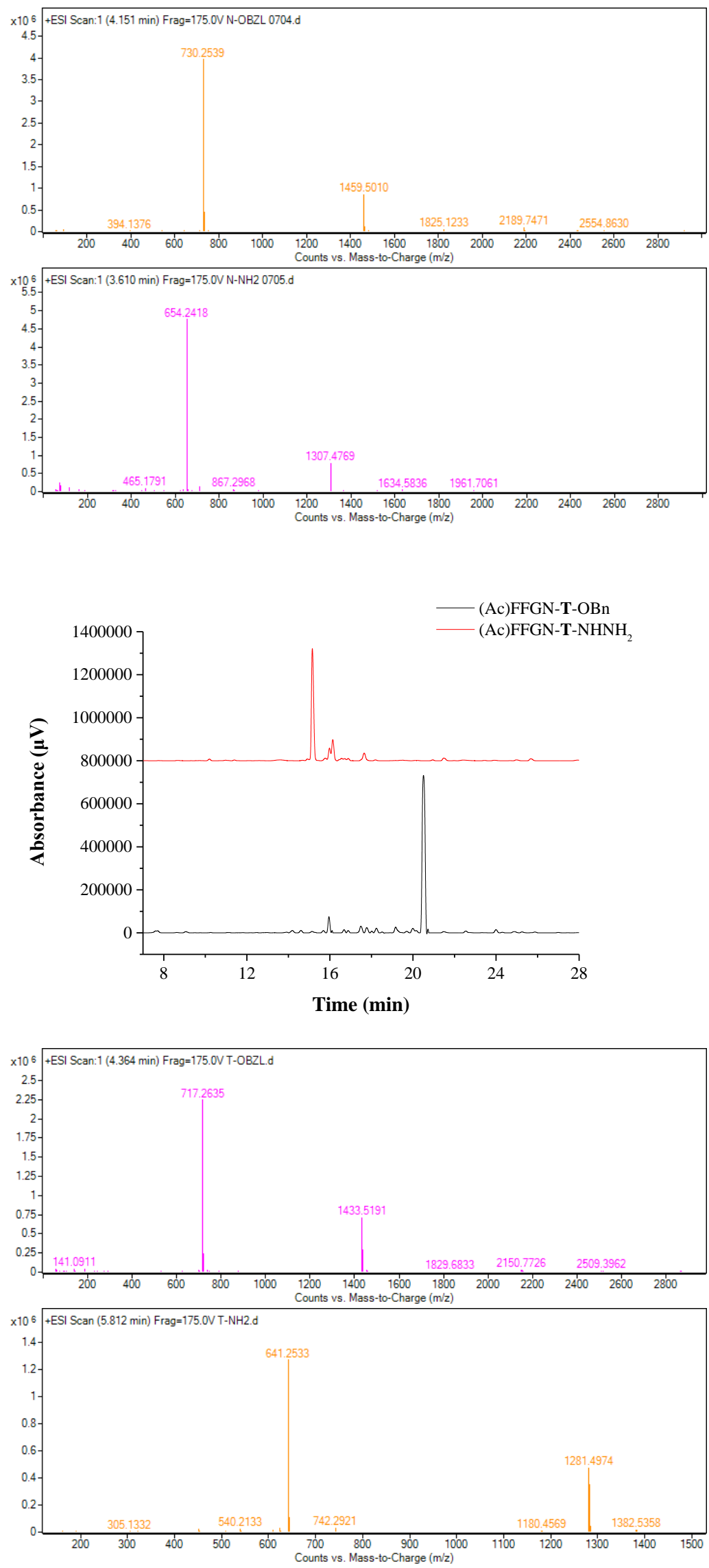

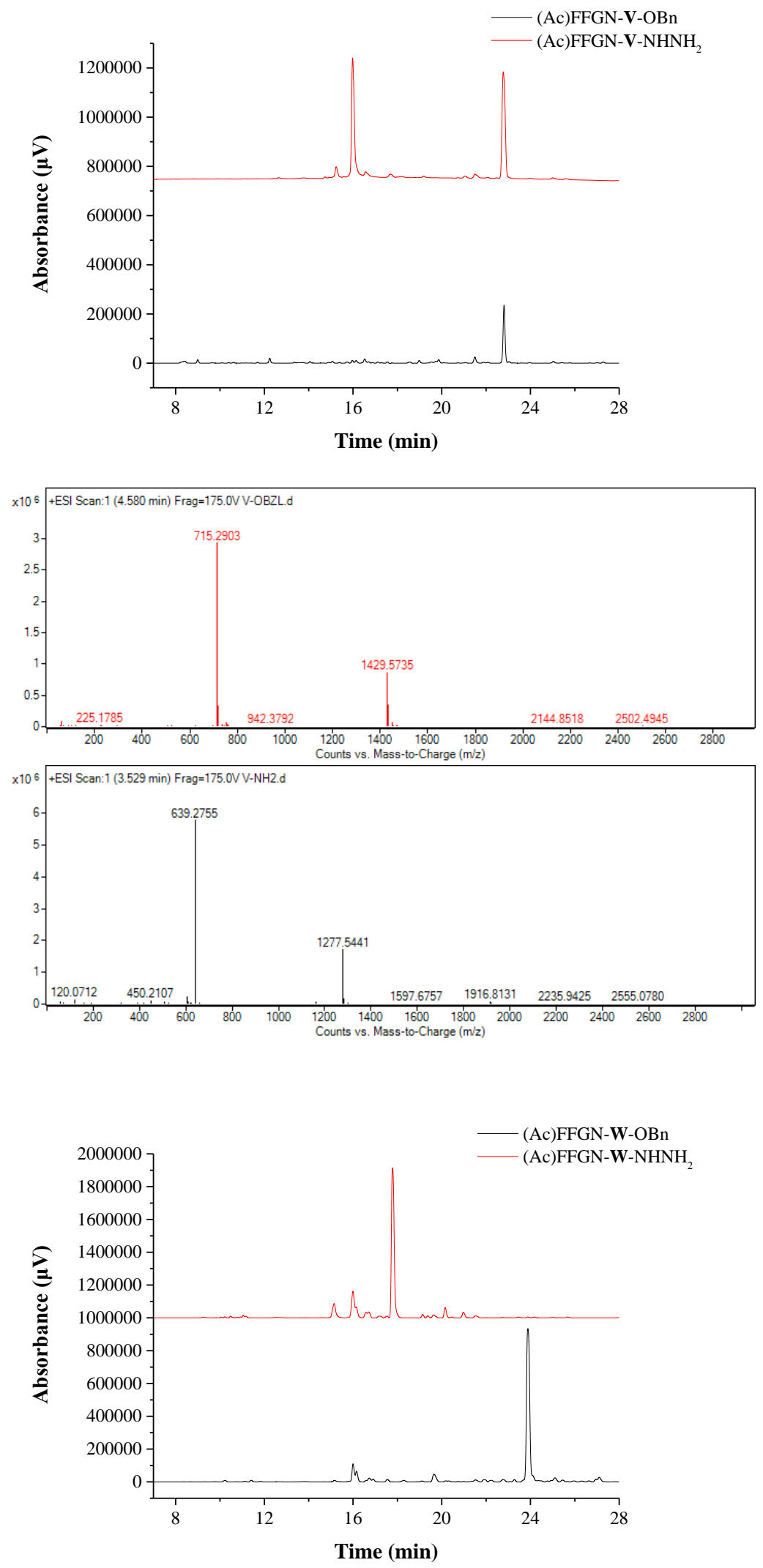

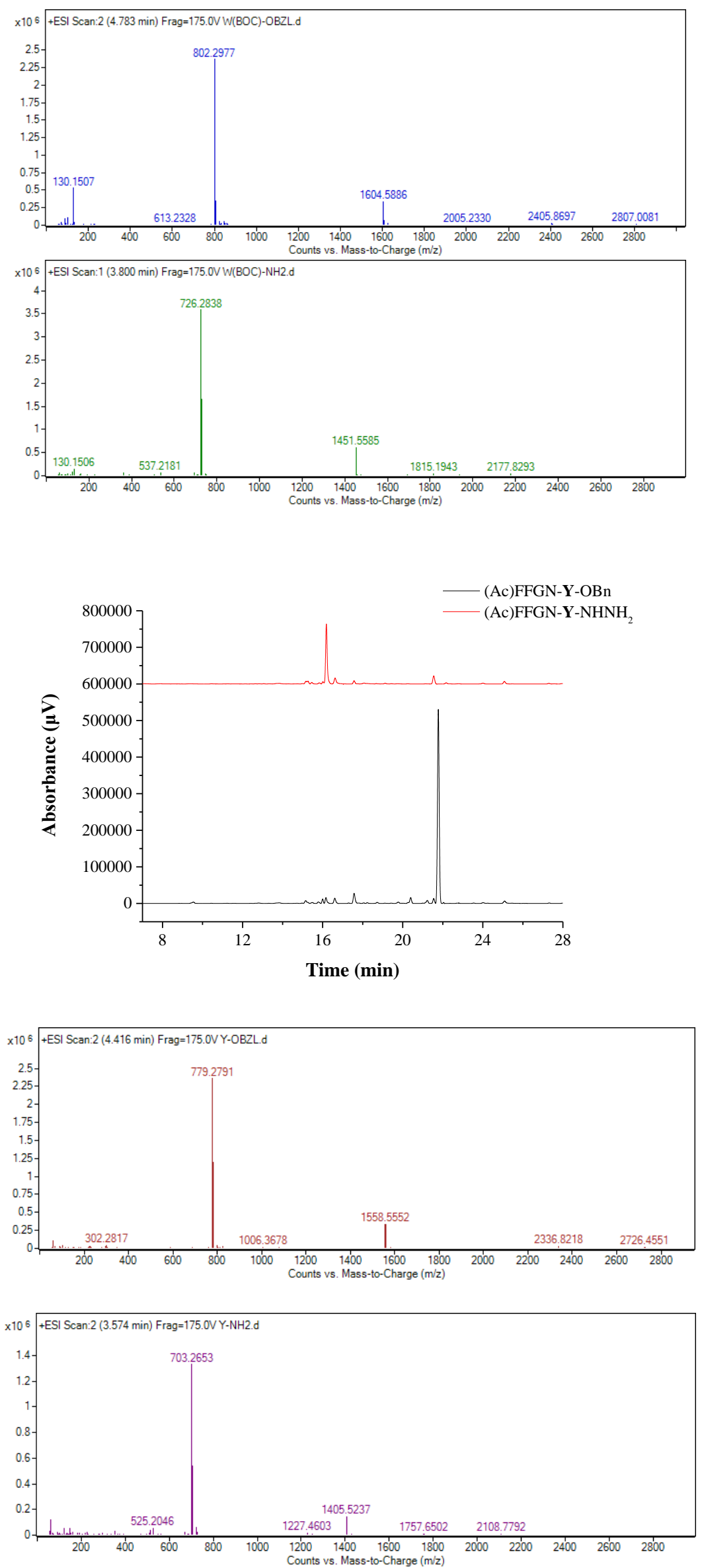

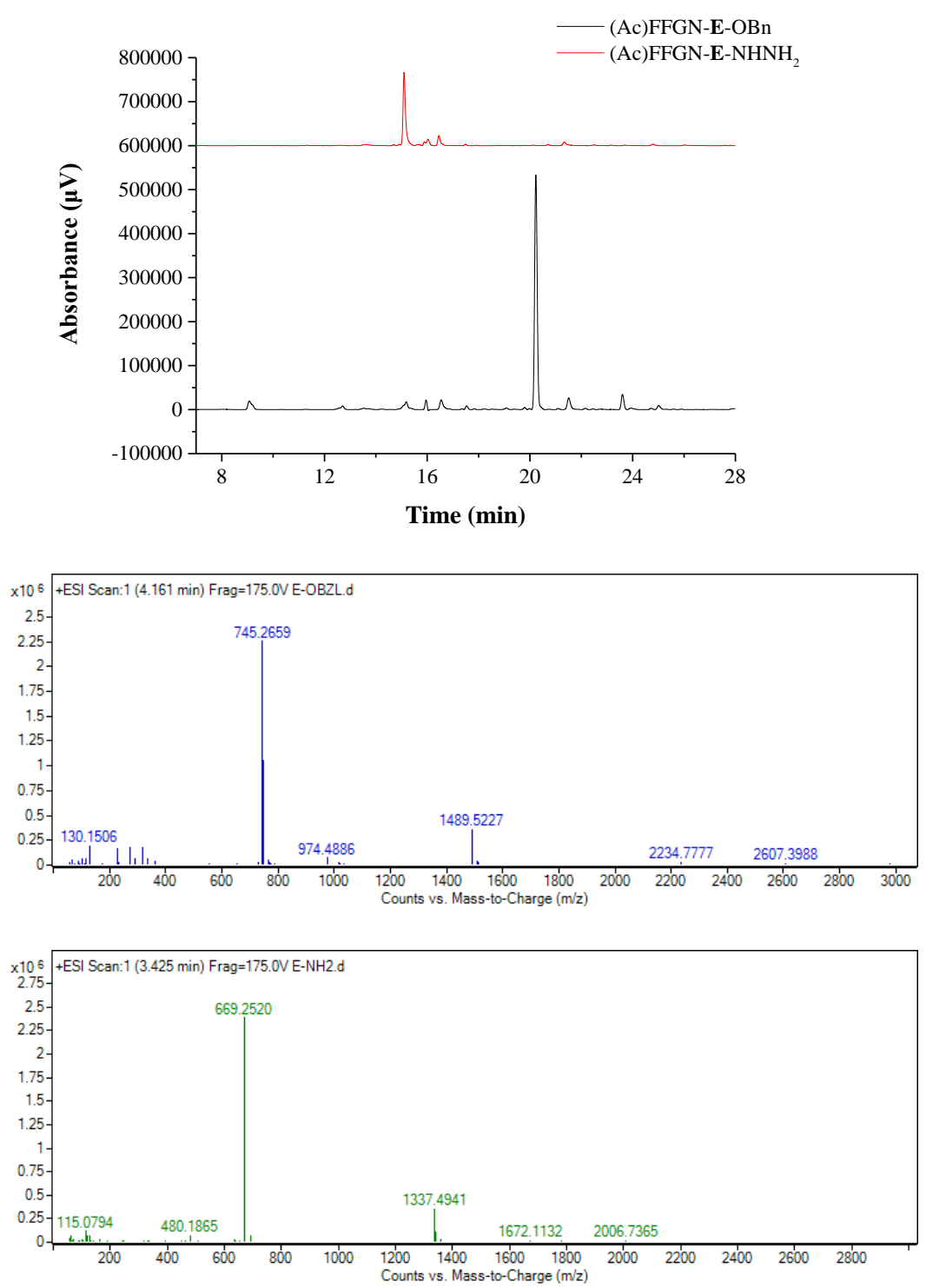

\section{Survey on the hydrazinolysis efficiency of bulky AA}

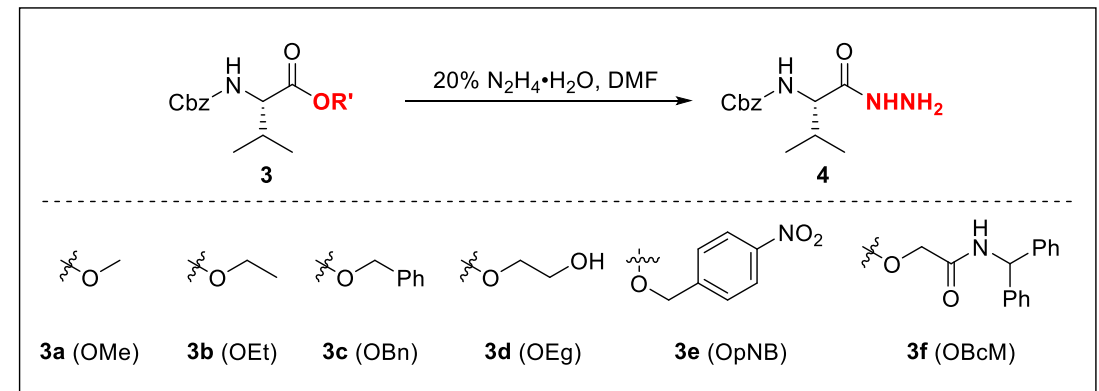

\section{Synthesis of Cbz-V-OR'}

$\mathrm{N}$-Cbz-L-valine (250mg, 1mmol, 1.2 equiv), alcohol (1.2mmol, 1.2 equiv), DCC (1.1 $\mathrm{mmol}, 227 \mathrm{mg}$, 1.1 equiv) and DMAP $(0.1 \mathrm{mmol}, 12.2 \mathrm{mg}, 0.1$ equiv) were added into DCM and stirred until the reaction was finished as monitored by TLC (EA/PE 1:2). The solid was removed by filtration and the filtrate was concentrated to dryness and purified by flash chromatography (PE/EA 2:1) to give the corresponding products. 
<smiles>COC(=O)[C@H](NC(=O)OCc1ccccc1)C(C)C</smiles>

methyl ((benzyloxy)carbonyl)- $L$-valinate

Cbz-V-OMe (3a) was prepared as a yellow oil (190.9 mg) in $82 \%$ yield.

${ }^{1} \mathrm{H}$ NMR $\left(500 \mathrm{MHz}, \mathrm{CDCl}_{3}\right) \delta 7.39-7.29(\mathrm{~m}, 5 \mathrm{H}), 5.11(\mathrm{~s}, 2 \mathrm{H}), 4.31(\mathrm{dd}, J=9.0,4.8 \mathrm{~Hz}, 1 \mathrm{H}), 3.74$ (s, 3H), $2.16(\mathrm{td}, J=13.3,6.6 \mathrm{~Hz}, 1 \mathrm{H}), 0.97(\mathrm{~d}, J=6.8 \mathrm{~Hz}, 3 \mathrm{H}), 0.89$ (d, $J=6.9 \mathrm{~Hz}, 3 \mathrm{H}) .{ }^{13} \mathrm{C}$ NMR $\left(126 \mathrm{MHz}, \mathrm{CDCl}_{3}\right) \delta 172.5,156.2,136.2,128.5,128.2,128.1,67.0,59.0,52.1,31.3,18.9,17.5$.

ESI-MS, m/z: $[\mathrm{M}+\mathrm{H}]^{+}$calcd for $\mathrm{C}_{14} \mathrm{H}_{20} \mathrm{NO}_{4}{ }^{+} 266.1378$; found 266.1428

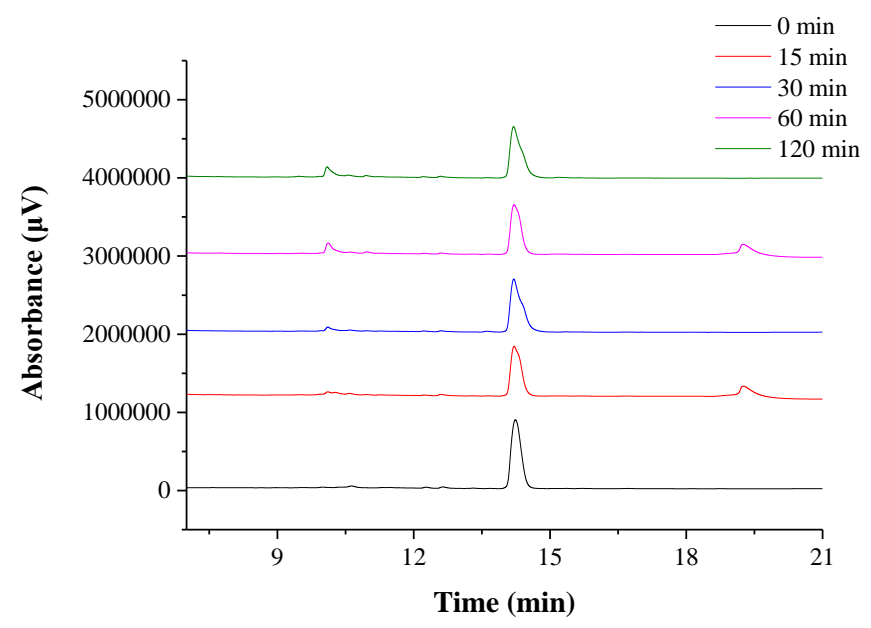

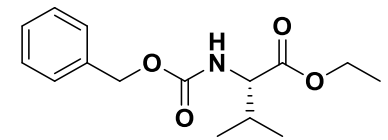

ethyl ((benzyloxy)carbonyl)- $L$-valinate

Cbz-V-OEt (3b) was prepared as a yellow oil (220.3 mg) in $79 \%$ yield.

${ }^{1} \mathrm{H}$ NMR $\left(500 \mathrm{MHz}, \mathrm{CDCl}_{3}\right) \delta 7.39-7.30(\mathrm{~m}, 5 \mathrm{H}), 5.11(\mathrm{~s}, 2 \mathrm{H}), 4.29(\mathrm{dd}, J=9.1,4.7 \mathrm{~Hz}, 1 \mathrm{H}), 4.20$ $(\mathrm{td}, J=6.8,3.7 \mathrm{~Hz}, 2 \mathrm{H}), 2.16(\mathrm{dt}, J=11.7,6.8 \mathrm{~Hz}, 1 \mathrm{H}), 1.28(\mathrm{t}, J=7.1 \mathrm{~Hz}, 3 \mathrm{H}), 0.97(\mathrm{~d}, J=6.9$ $\mathrm{Hz}, 3 \mathrm{H}), 0.89(\mathrm{~d}, J=6.9 \mathrm{~Hz}, 3 \mathrm{H}) .{ }^{13} \mathrm{C} \mathrm{NMR}\left(126 \mathrm{MHz}, \mathrm{CDCl}_{3}\right) \delta 171.8,156.0,136.0,128.3,127.9$, 127.9, 77.0, 66.7, 61.0, 58.7, 31.1, 18.7, 17.2, 14.0.

ESI-MS, m/z: $[\mathrm{M}+\mathrm{H}]^{+}$calcd for $\mathrm{C}_{15} \mathrm{H}_{22} \mathrm{NO}_{4}{ }^{+} 280.1578$; found 280.1538 . 


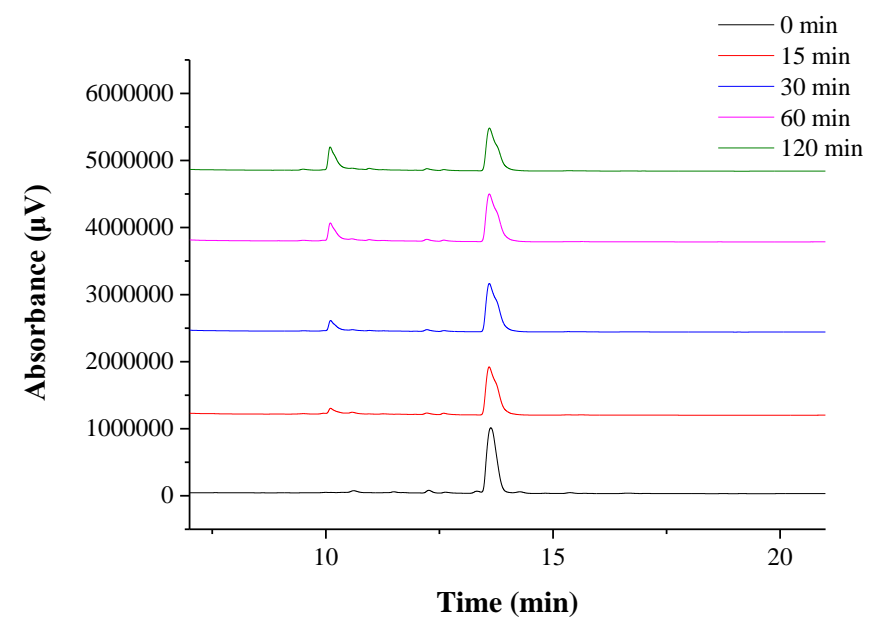

Q

benzyl ((benzyloxy)carbonyl)- $L$-valinate

Cbz-V-OBn(3c) was prepared as a yellow oil (262.3 mg) in $77 \%$ yield.

${ }^{1} \mathrm{H}$ NMR $\left(500 \mathrm{MHz}, \mathrm{CDCl}_{3}\right) \delta 7.40-7.28(\mathrm{~m}, 10 \mathrm{H}), 5.17(\mathrm{dd}, J=25.0,12.2 \mathrm{~Hz}, 2 \mathrm{H}), 5.11(\mathrm{~s}, 2 \mathrm{H})$, 4.36 (dd, $J=9.1,4.6 \mathrm{~Hz}, 1 \mathrm{H}), 2.18(\mathrm{td}, J=13.4,6.7 \mathrm{~Hz}, 1 \mathrm{H}), 0.95(\mathrm{~d}, J=6.8 \mathrm{~Hz}, 3 \mathrm{H}), 0.85$ (d, $J=$ $6.9 \mathrm{~Hz}, 3 \mathrm{H}) .{ }^{13} \mathrm{C} \mathrm{NMR}\left(126 \mathrm{MHz}, \mathrm{CDCl}_{3}\right) \delta 171.9,156.5,136.2,135.3,128.6,128.5,128.4,128.4$, 128.2, 128.1, 77.2, 67.0, 59.0, 31.3, 18.9, 17.4.

ESI-MS, m/z: $[\mathrm{M}+\mathrm{H}]+$ calcd for $\mathrm{C}_{20} \mathrm{H}_{24} \mathrm{NO}_{4}{ }^{+} 342.1687$; found 342.1679 .
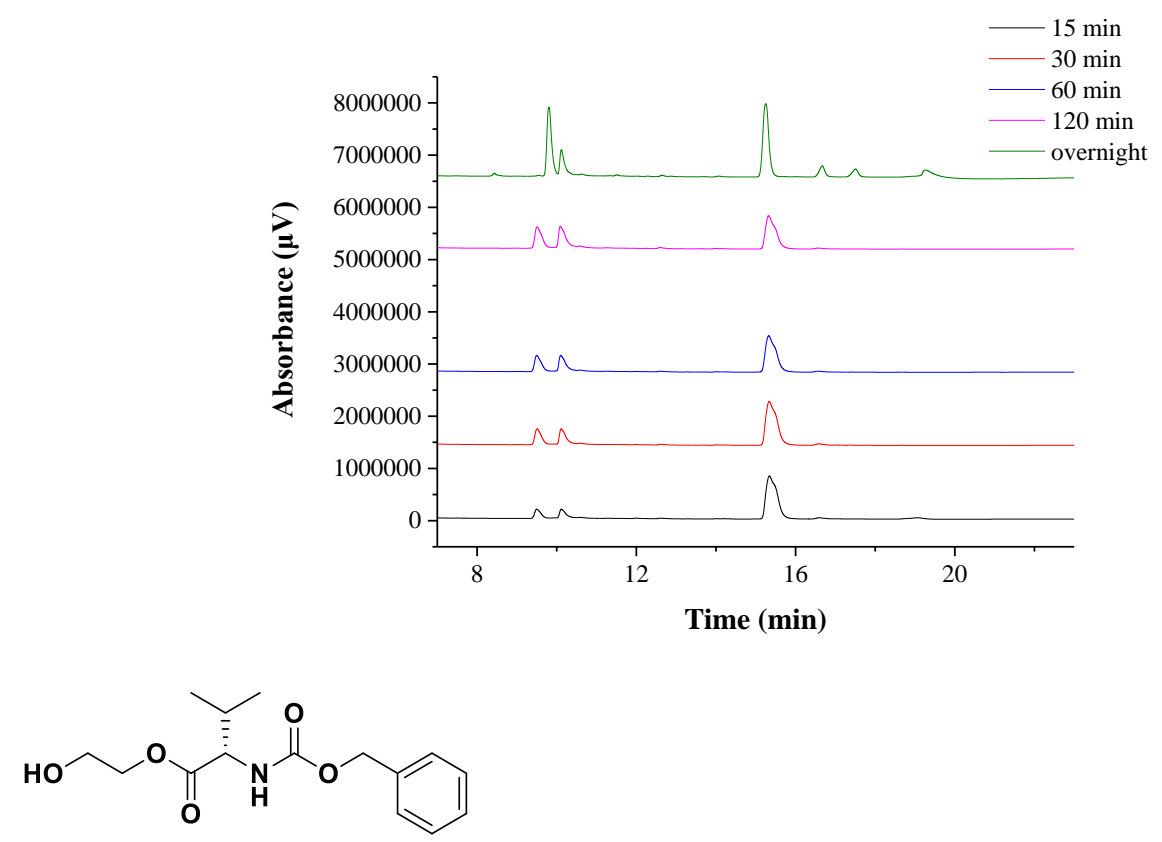

\section{2-hydroxyethyl ((benzyloxy)carbonyl)- $L$-valinate}

Cbz-V-OEg (3d) was prepared as a yellow oil (200.7 mg) in $68 \%$ yield.

${ }^{1} \mathrm{H}$ NMR $\left(500 \mathrm{MHz}, \mathrm{CDCl}_{3}\right) \delta 7.46-7.28(\mathrm{~m}, 5 \mathrm{H}), 5.34(\mathrm{~d}, J=8.4 \mathrm{~Hz}, 1 \mathrm{H}), 5.14(\mathrm{~s}, 2 \mathrm{H}), 4.31(\mathrm{dd}$, $J=8.9,4.7 \mathrm{~Hz}, 2 \mathrm{H}), 3.85(\mathrm{t}, J=4.4 \mathrm{~Hz}, 2 \mathrm{H}), 2.21(\mathrm{dt}, J=19.1,6.9 \mathrm{~Hz}, 1 \mathrm{H}), 1.02(\mathrm{~d}, J=6.7 \mathrm{~Hz}$, 
$3 \mathrm{H}), 0.95(\mathrm{~d}, J=6.9 \mathrm{~Hz}, 3 \mathrm{H}) .{ }^{13} \mathrm{C} \mathrm{NMR}\left(126 \mathrm{MHz}, \mathrm{CDCl}_{3}\right) \delta 172.3,156.4,136.1,128.5,128.2$, 128.1, 67.2, 66.6, 60.9, 59.3, 31.0, 19.0, 17.6.

ESI-MS, m/z: $[\mathrm{M}+\mathrm{H}]+$ calcd for $\mathrm{C}_{15} \mathrm{H}_{22} \mathrm{NO}_{5}{ }^{+} 296.1478$; found 296.1469.

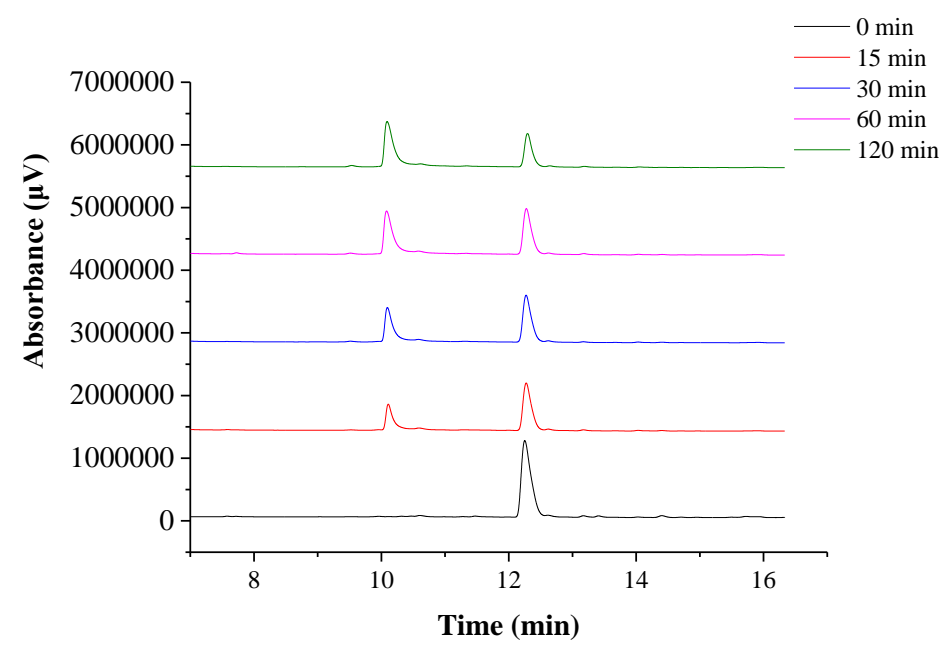<smiles>CC(C)[C@H](NC(=O)OCc1ccccc1)C(=O)OCc1ccc([N+](=O)[O-])cc1</smiles>

\section{4-nitrobenzyl ((benzyloxy)carbonyl)-L-valinate}

Cbz-V-OpNB (3e) was prepared as a yellow oil $(220.1 \mathrm{mg})$ in $57 \%$ yield.

${ }^{1} \mathrm{H}$ NMR $\left(500 \mathrm{MHz}, \mathrm{CDCl}_{3}\right) \delta 8.22(\mathrm{~d}, J=8.4 \mathrm{~Hz}, 2 \mathrm{H}), 7.51(\mathrm{~d}, J=8.3 \mathrm{~Hz}, 2 \mathrm{H}), 7.37-7.32(\mathrm{~m}$, $5 \mathrm{H}), 5.26(\mathrm{~s}, 2 \mathrm{H}), 5.12(\mathrm{~s}, 2 \mathrm{H}), 4.37(\mathrm{dd}, J=9.0,4.8 \mathrm{~Hz}, 1 \mathrm{H}), 2.20(\mathrm{td}, J=13.2,6.6 \mathrm{~Hz}, 1 \mathrm{H}), 0.98$ $(\mathrm{d}, J=6.8 \mathrm{~Hz}, 3 \mathrm{H}), 0.88(\mathrm{~d}, J=6.9 \mathrm{~Hz}, 3 \mathrm{H}) .{ }^{13} \mathrm{C} \mathrm{NMR}\left(126 \mathrm{MHz}, \mathrm{CDCl}_{3}\right) \delta 171.8,156.2,147.8$, 142.4, 136.1, 128.6, 128.5, 128.1, 128.1, 123.8, 77.2, 67.2, 65.4, 59.1, 31.1, 19.0, 17.5.

ESI-MS, m/z: $[\mathrm{M}+\mathrm{H}]+$ calcd for $\mathrm{C}_{20} \mathrm{H}_{23} \mathrm{~N}_{2} \mathrm{O}_{6}{ }^{+} 386.1500$; found 386.1492 .

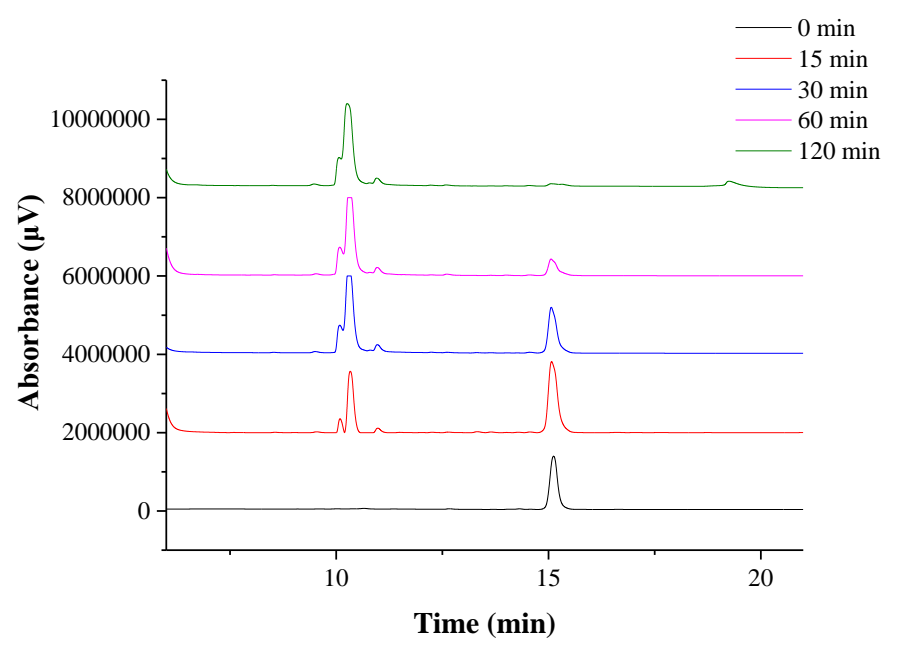


<smiles>CC(C)C(NC(=O)OCc1ccccc1)C(=O)OCC(=O)NC(c1ccccc1)c1ccccc1</smiles>

2-(benzhydrylamino)-2-oxoethyl ((benzyloxy)carbonyl)- $L$-valinate

Cbz-V-OBcM (3f) was prepared as a yellow oil $(327.1 \mathrm{mg})$ in $69 \%$ yield.

${ }^{1} \mathrm{H}$ NMR $\left(500 \mathrm{MHz}, \mathrm{CDCl}_{3}\right) \delta 7.56(\mathrm{~d}, J=8.6 \mathrm{~Hz}, 1 \mathrm{H}), 7.36-7.25(\mathrm{~m}, 10 \mathrm{H}), 7.21(\mathrm{dd}, J=16.3$, $7.5 \mathrm{~Hz}, 5 \mathrm{H}), 6.38(\mathrm{~d}, J=8.7 \mathrm{~Hz}, 1 \mathrm{H}), 5.16(\mathrm{~d}, J=6.5 \mathrm{~Hz}, 1 \mathrm{H}), 4.84-4.81(\mathrm{~m}, 2 \mathrm{H}), 4.60-4.56(\mathrm{~m}$, $2 \mathrm{H}), 4.08(\mathrm{t}, J=6.5 \mathrm{~Hz}, 1 \mathrm{H}), 2.12(\mathrm{dq}, J=13.5,6.8 \mathrm{~Hz}, 1 \mathrm{H}), 0.99(\mathrm{~d}, J=6.8 \mathrm{~Hz}, 3 \mathrm{H}), 0.96(\mathrm{~d}, J=$ $6.8 \mathrm{~Hz}, 3 \mathrm{H}) .{ }^{13} \mathrm{C}$ NMR $\left(126 \mathrm{MHz}, \mathrm{CDCl}_{3}\right) \delta 171.6,166.1,156.7,141.3,141.4,135.7,128.6,128.5$, 128.4, 128.3, 128.1, 127.8, 127.6, 127.5, 127.3, 67.3, 63.0, 60.1, 56.3, 30.1, 19.0, 18.2.

ESI-MS, m/z: $[\mathrm{M}+\mathrm{H}]+$ calcd for $\mathrm{C}_{28} \mathrm{H}_{31} \mathrm{~N}_{2} \mathrm{O}_{5}{ }^{+} 475.2278$; found 475.2318 .

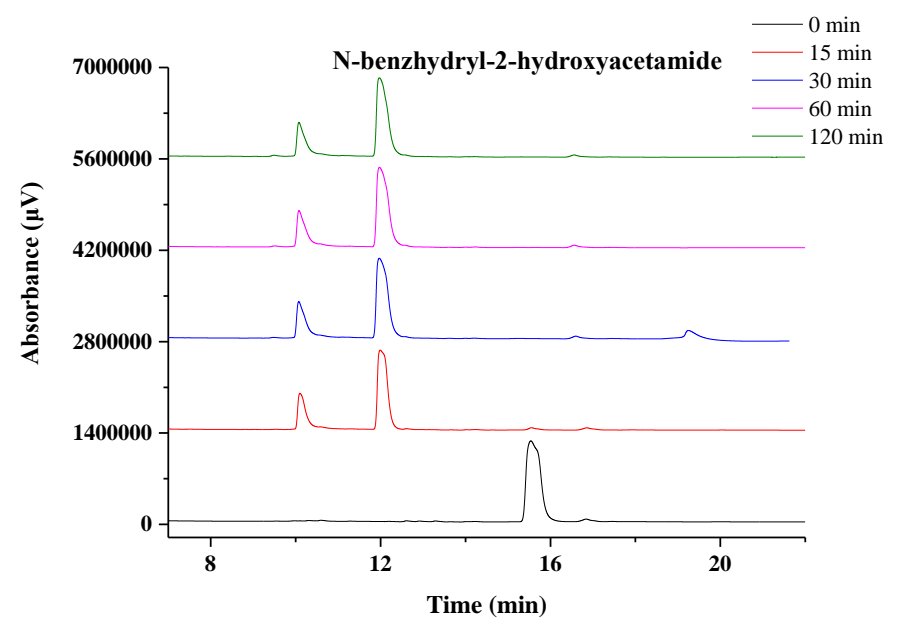

\section{Cbz-V-NHNH}<smiles>CC(C)C(NC(=O)OCc1ccccc1)C(=O)NN</smiles>

benzyl (S)-(1-hydrazineyl-3-methyl-1-oxobutan-2-yl)carbamate

Cbz-V-NHNH 2 was prepared as a yellow oil $(235.9 \mathrm{mg})$ in $89 \%$ yield.

${ }^{1} \mathrm{H}$ NMR (500 MHz, Methanol- $\left.d_{4}\right) \delta 7.42-7.26(\mathrm{~m}, 5 \mathrm{H}), 5.12-5.03(\mathrm{~m}, 2 \mathrm{H}), 3.83(\mathrm{~d}, J=7.6 \mathrm{~Hz}$, $1 \mathrm{H}), 2.00(\mathrm{dt}, J=13.8,6.9 \mathrm{~Hz}, 1 \mathrm{H}), 0.93(\mathrm{t}, J=6.7 \mathrm{~Hz}, 6 \mathrm{H}) .{ }^{13} \mathrm{C}$ NMR $(126 \mathrm{MHz}, \mathrm{MeOD}) \delta 171.8$, 157.1, 136.1, 128.0, 127.6, 127.4, 66.2, 59.5, 48.0, 47.8, 47.7, 30.5, 18.1, 17.3.

ESI-MS, m/z: $[\mathrm{M}+\mathrm{H}]+$ calcd for $\mathrm{C}_{13} \mathrm{H}_{20} \mathrm{~N}_{3} \mathrm{O}_{3}{ }^{+} 266.1478$; found 266.1522 . 


\section{Research on the extent of racemization}

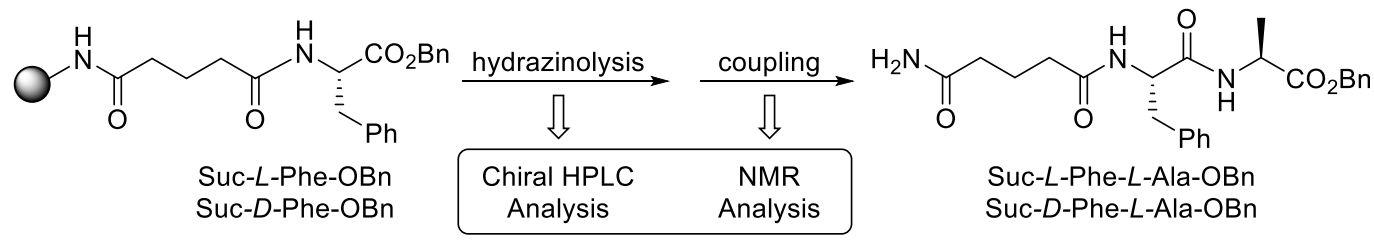

Scheme S1. Inverse solid-phase peptide synthesis of Suc- $L / D-P h e-L-A l a-O B n$.

First, the evaluation of racemization was carried out on the hydrazinolysis of $(D)$ or $(L)$-Phe-OBn by treatment of $20 \%$ hydrazine at room temperature for 24 hours. Chiral HPLC analysis data showed that there was no signal of $(D)-\mathrm{Phe}^{-\mathrm{NHNH}_{2}}$ in Figure S1c, which indicated no racemization

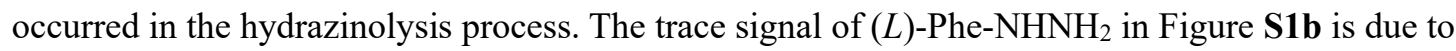
the purity of raw materials of $(D)$-Phe-OBn which contains about $5 \%(L)$-Phe-OBn.

(a)
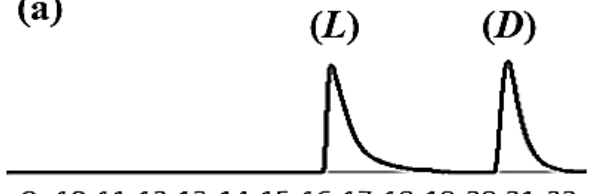

$9 \quad 10111213141516171819202122$ Retention time(min)

(b)

$(L)$

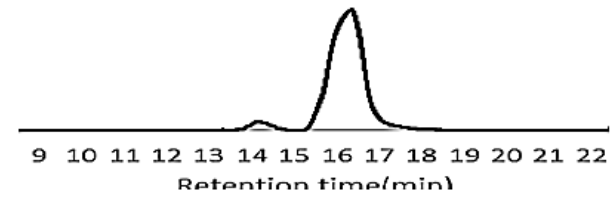

(c)

(D)

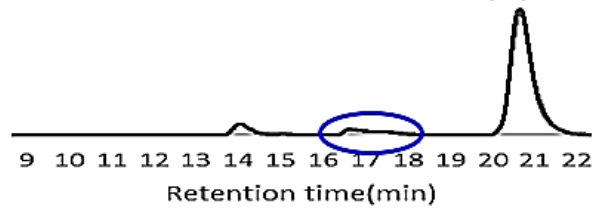

Figure S1 Chiral HPLC analysis of hydrazinolysis of Suc-Phe-OBn

Following the hydrazinolysis process, Azide-coupling was conducted to afford the dipeptide products. NMR analysis demonstrated there was apparent difference (3.2 ppm to $2.6 \mathrm{ppm}$ ) between the diastereomers. Absence of NMR response of Suc- $D$-Phe- $L$-Ala-OBn in Figure $\mathbf{S 2} \mathbf{b}$ indicated no racemization occurring in coupling process, and trace response of Suc- $L$-Phe- $L$-Ala-OBn in Figure S1a was consistent with Figure S1b. 


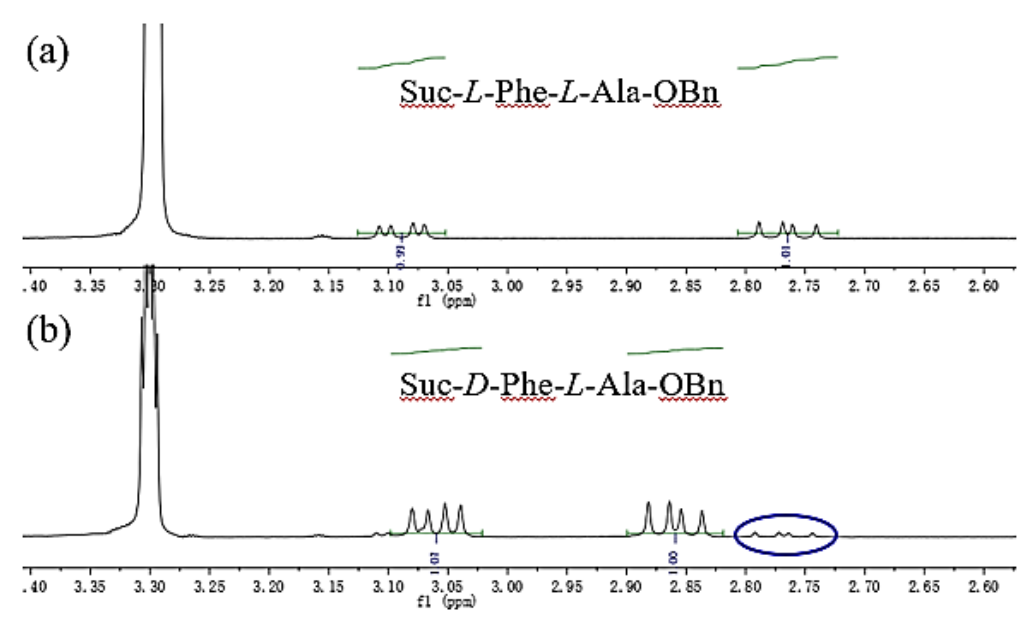

Figure S2 NMR analysis of Suc-Phe-Ala-OBn<smiles>CCCCCC(=O)C(C)NC(=O)[C@H](Cc1ccccc1)NC(=O)CCCC(N)=O</smiles>

Suc-L-Phe-L-Ala-OBn

According to the general methods of Azide-ISPPS, Suc- $L$-Phe- $L$-Ala-OBn was prepared as a white powder $(91.0 \mathrm{mg})$ in $83 \%$ yield.

${ }^{1} \mathrm{H}$ NMR $\left(500 \mathrm{MHz}\right.$, Methanol- $\left.d_{4}\right) \delta 8.51(\mathrm{~d}, J=7.2 \mathrm{~Hz}, 1 \mathrm{H}), 8.08(\mathrm{~d}, J=8.2 \mathrm{~Hz}, 1 \mathrm{H}), 7.43-7.29$ $(\mathrm{m}, 5 \mathrm{H}), 7.28-7.12(\mathrm{~m}, 5 \mathrm{H}), 5.16(\mathrm{~d}, J=12.3 \mathrm{~Hz}, 1 \mathrm{H}), 5.13(\mathrm{~d}, J=12.3 \mathrm{~Hz}, 1 \mathrm{H}), 4.65(\mathrm{ddd}, J=$ 9.9, 8.1, 4.8 Hz, 1H), 4.45 (p, $J=7.2 \mathrm{~Hz}, 1 \mathrm{H}), 3.09$ (dd, $J=14.1,4.8 \mathrm{~Hz}, 1 \mathrm{H}), 2.76$ (dd, $J=14.1$, $10.0 \mathrm{~Hz}, 1 \mathrm{H}), 2.16$ (td, $J=7.3,2.9 \mathrm{~Hz}, 2 \mathrm{H}), 2.06(\mathrm{dd}, J=8.1,7.0 \mathrm{~Hz}, 2 \mathrm{H}), 1.75(\mathrm{p}, J=7.5 \mathrm{~Hz}, 2 \mathrm{H})$, $1.39(\mathrm{~d}, J=7.3 \mathrm{~Hz}, 3 \mathrm{H})$.

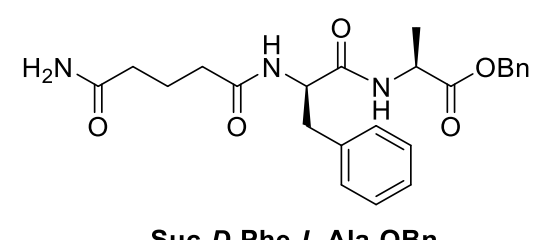

According to the general methods of Azide-ISPPS, Suc-D-Phe- $L$-Ala-OBn was prepared as a white powder $(94.3 \mathrm{mg})$ in $86 \%$ yield.

${ }^{1} \mathrm{H}$ NMR $\left(500 \mathrm{MHz}\right.$, Methanol- $\left.d_{4}\right) \delta 7.36-7.17(\mathrm{~m}, 10 \mathrm{H}), 5.15(\mathrm{~d}, J=12.4 \mathrm{~Hz}, 1 \mathrm{H}), 5.12(\mathrm{~d}, J=$ $12.4 \mathrm{~Hz}, 1 \mathrm{H}), 4.66(\mathrm{dd}, J=8.7,6.6 \mathrm{~Hz}, 1 \mathrm{H}), 4.36(\mathrm{q}, J=7.3 \mathrm{~Hz}, 1 \mathrm{H}), 3.06(\mathrm{dd}, J=13.7,6.6 \mathrm{~Hz}$, $1 \mathrm{H}), 2.86(\mathrm{dd}, J=13.7,8.7 \mathrm{~Hz}, 1 \mathrm{H}), 2.23-2.13(\mathrm{~m}, 2 \mathrm{H}), 2.09$ (td, $J=7.3,1.5 \mathrm{~Hz}, 2 \mathrm{H}), 1.77$ (p, $J$ $=7.5 \mathrm{~Hz}, 2 \mathrm{H}), 1.26(\mathrm{~d}, J=7.3 \mathrm{~Hz}, 3 \mathrm{H})$. 
7. Evaluation of the efficiency by Fmoc-SPPS V.S. Acyl Azide-ISPPS

Fmoc-SPPS: Ac-FFGNSLV-NH 2

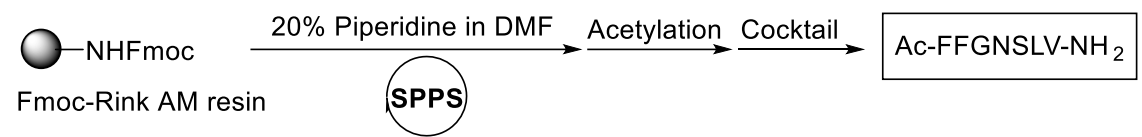

Scheme S2. Solid-phase peptide synthesis of Ac-FFGNSLV-NH 2 .

Ac-FFGNSLV-NH $\mathbf{N H}_{2}$ was prepared as a white powder in $99.6 \%$ yield from rink AM resin following the general procedure of Fmoc-SPPS.

ESI-MS, m/z: $[\mathrm{M}+\mathrm{H}]^{+}$calcd for $\mathrm{C}_{40} \mathrm{H}_{58} \mathrm{~N}_{9} \mathrm{O}_{10}{ }^{+}$824.4307; found 824.3017 .

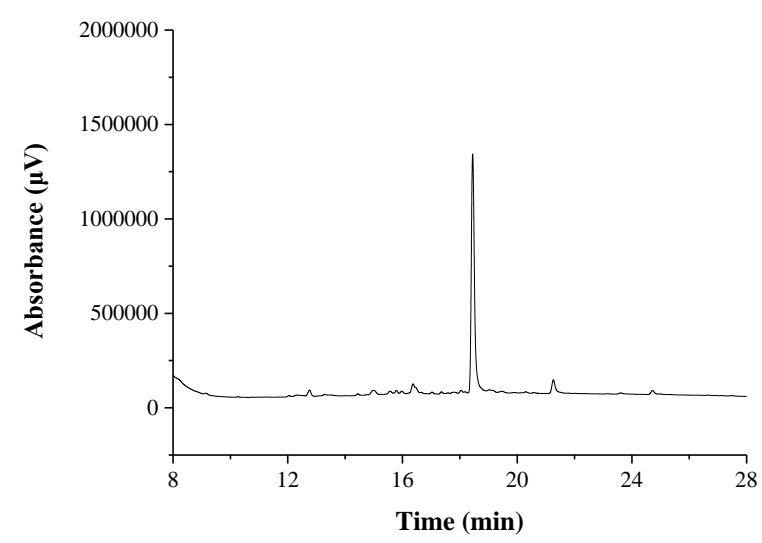

Azide-ISPPS: Ac-FFGNSLV-OH

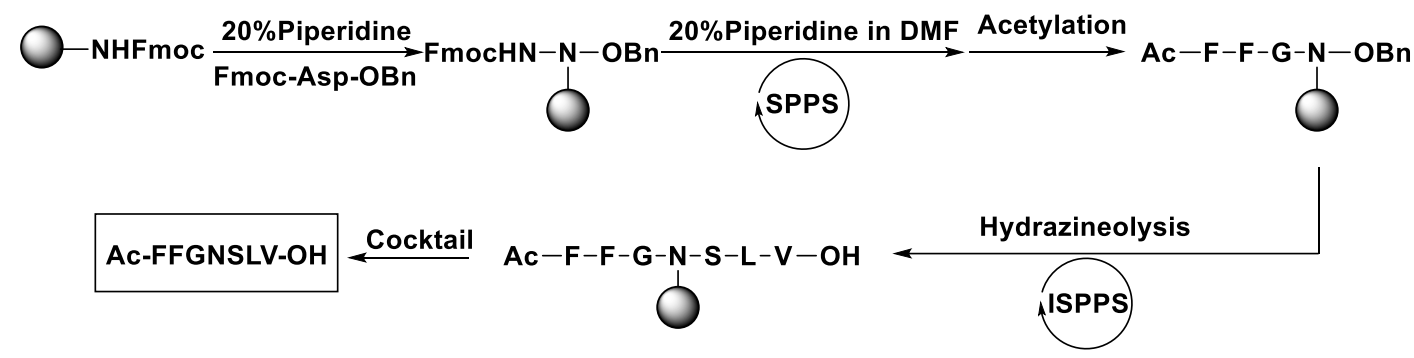

Scheme S3. Inverse solid-phase peptide synthesis of Ac-FFGNSLV-OH.

Ac-FFGNSLV-OH was prepared as a white powder in $98.4 \%$ yield from rink AM resin following the general procedures of Fmoc-SPPS and Azide-ISPPS.

ESI-MS, m/z: $[\mathrm{M}+\mathrm{H}]^{+}$calcd for $\mathrm{C}_{40} \mathrm{H}_{57} \mathrm{~N}_{8} \mathrm{O}_{11}{ }^{+}$825.4147; found 825.2666.

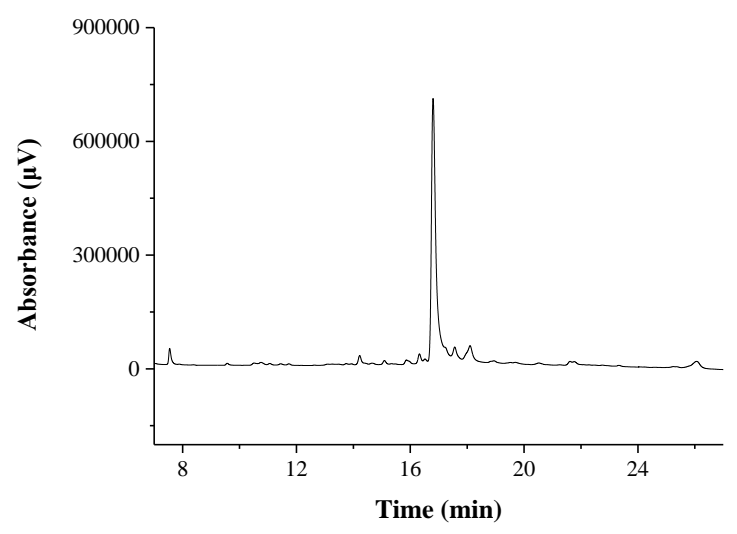




\section{Applications of this method}

a) synthesis of Ac-SLV-OH \& Suc-AAPL-pNA

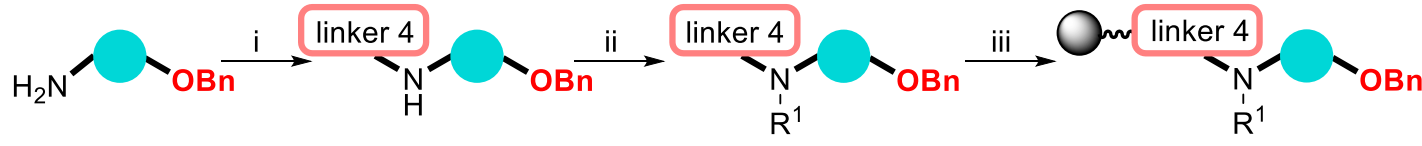

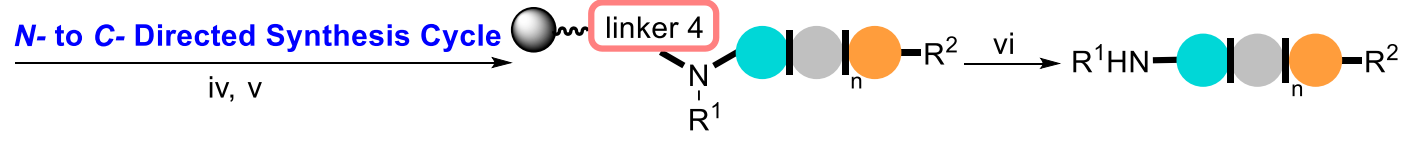

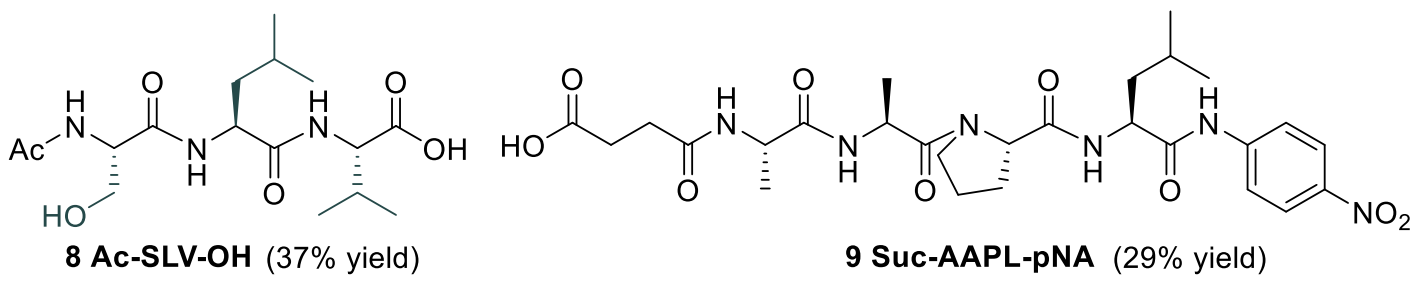

b) construction of peptides containing 20 AAs conbined with native chemical ligation

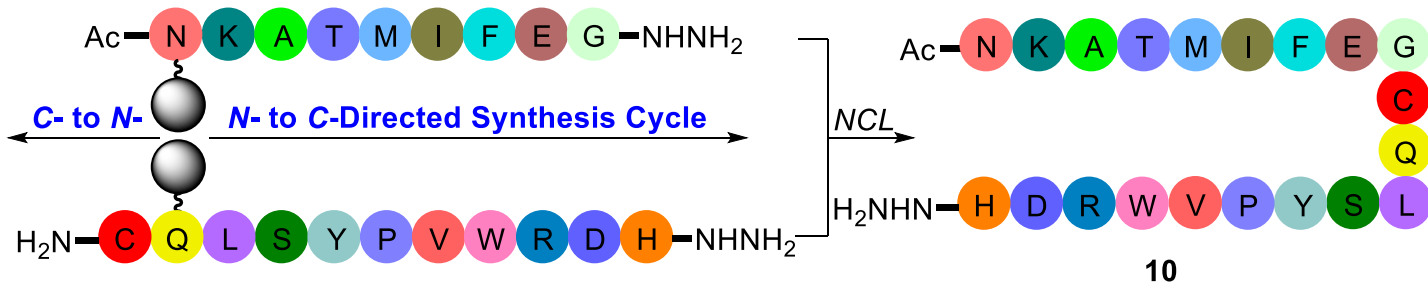

i) $\mathrm{NaBH}_{3} \mathrm{CN}, \mathrm{CH}_{3} \mathrm{COOH}, 78 \%$ yield. ii) For Ac-SLV-OH, acetic anhydride/pyridine, $96 \%$ yield. For SucAAPL-pNA, glutaric anhydride/pyridine, 92\% yield. iii) AM-resin, HATU/DIPEA. ISPPS cycle: iv) $20 \%$ $\mathrm{NH}_{2} \mathrm{NH}_{2}$ in DMF, rt. v) $\mathrm{NaNO}_{2}$ (10.0equiv), $\mathrm{pH}=3,-10{ }^{\circ} \mathrm{C}$; then AAs (5.0 equiv), DIPEA, $\mathrm{pH}=8$, rt. vi) Cock tail TFA $/ \mathrm{H}_{2} \mathrm{O} /$ Tips $(95: 2.5: 2.5)$.

a) Synthesis of two medicinal peptides

Compound 8 (Ac-SLV-OH) was prepared as a white powder in 37\% yield according to the procedure of Azide ISPPS.

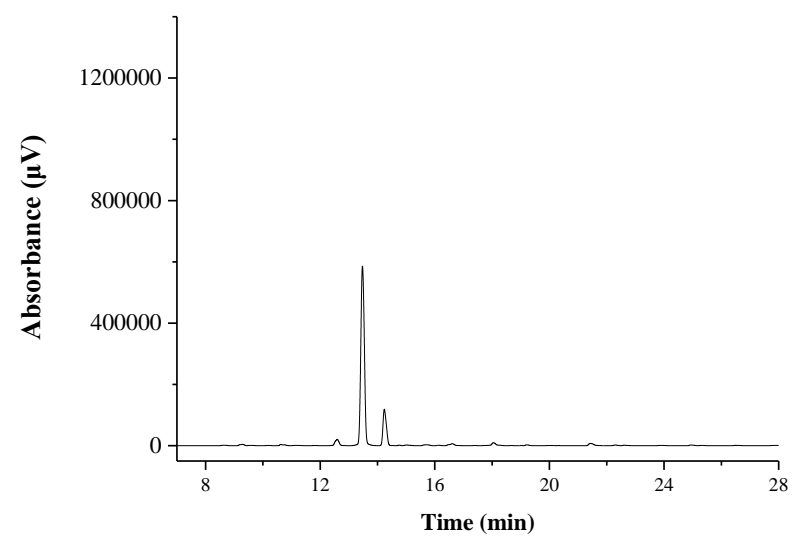




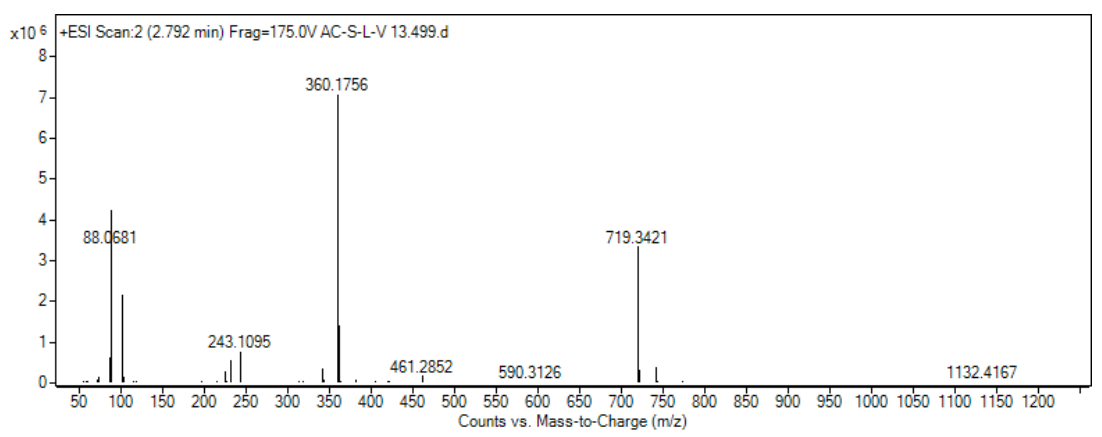

Compound 9 (Suc-AAPL-pNA) was prepared as a white powder in $29 \%$ yield according to the procedure of Azide ISPPS.
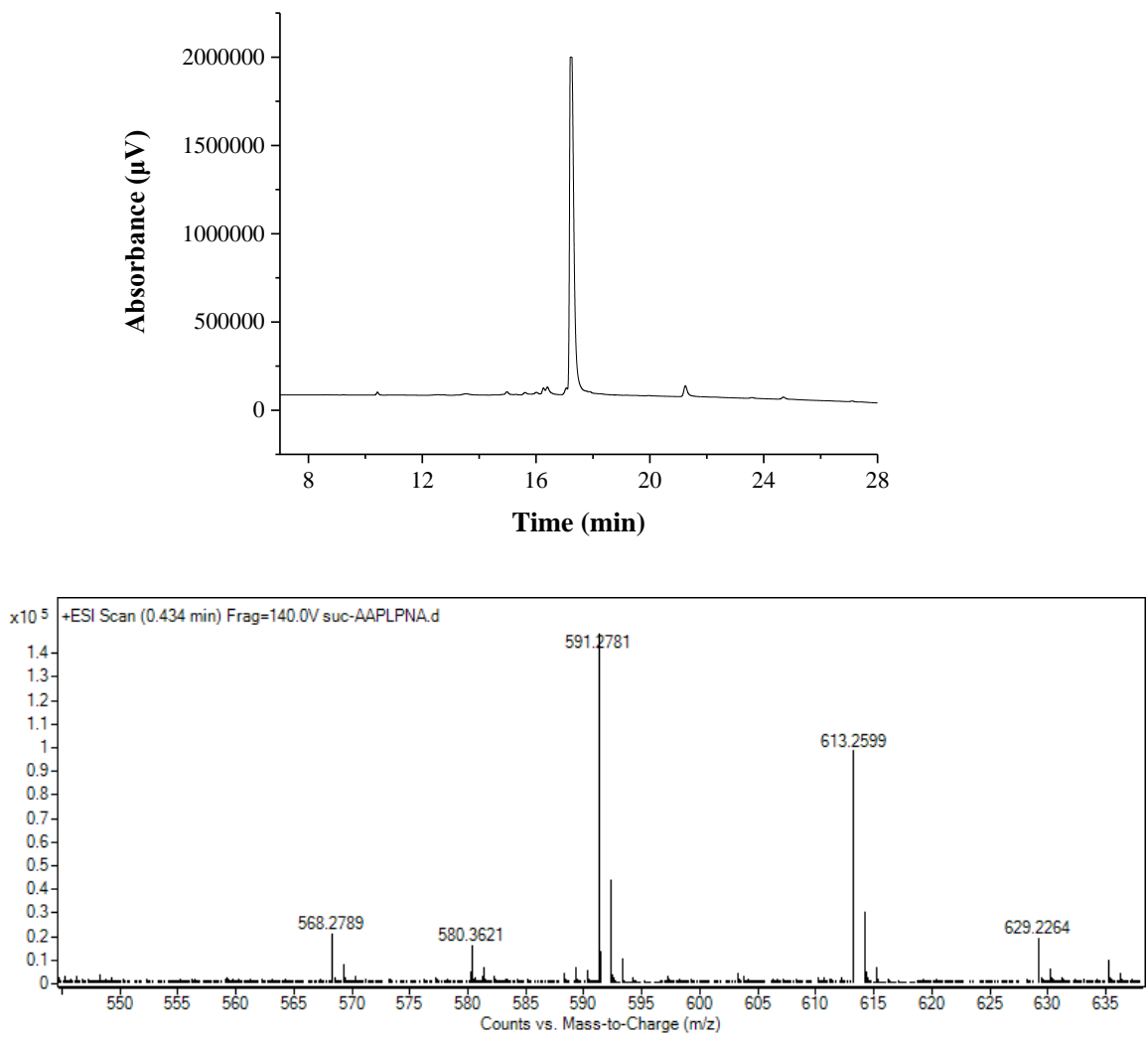

b) Synthesis of long-chain peptide (20 AAs) by Acyl Azide ISPPS combined with NCL Peptide A-OBcM 

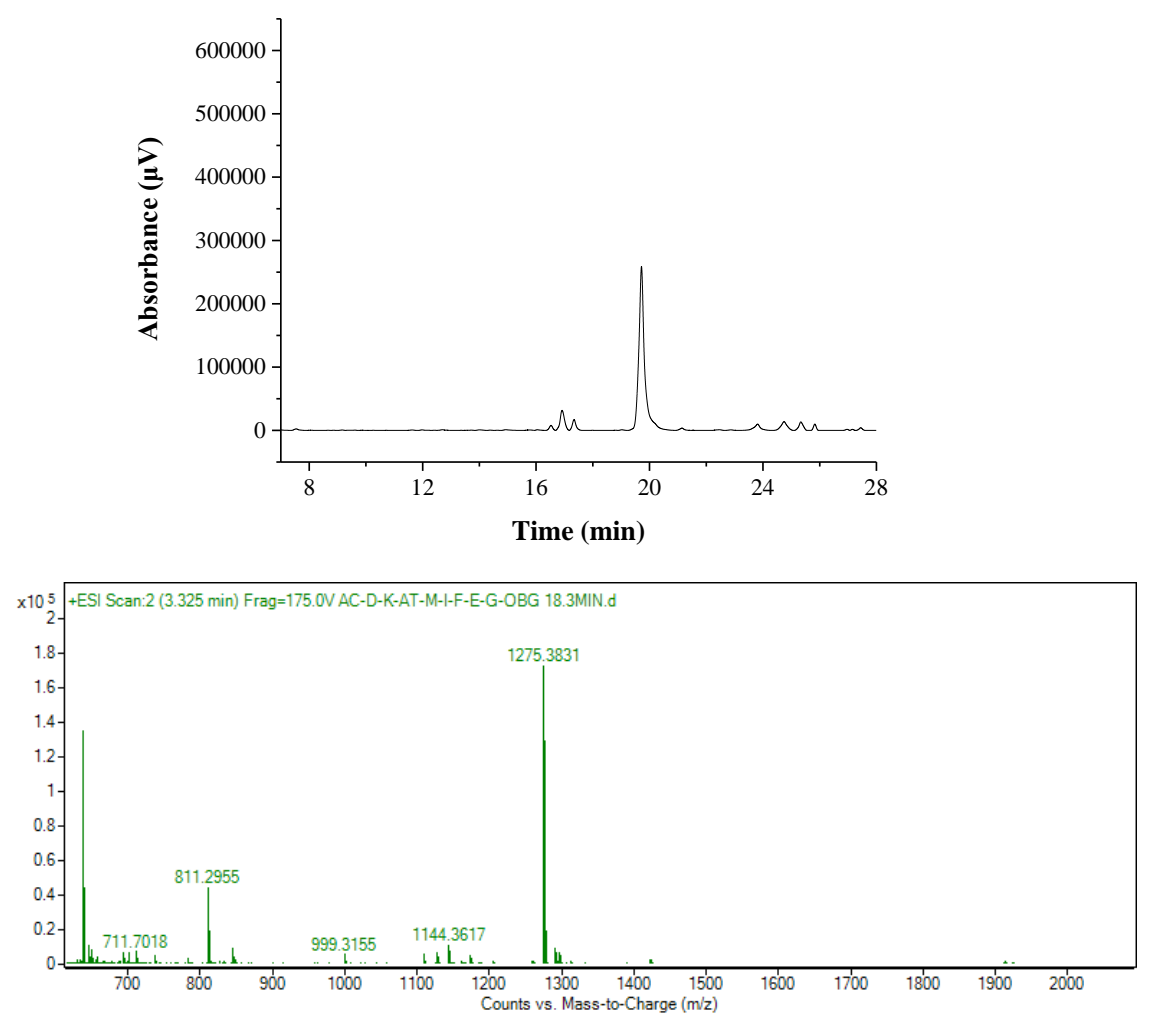

Peptide A-NHNH ${ }_{2}$
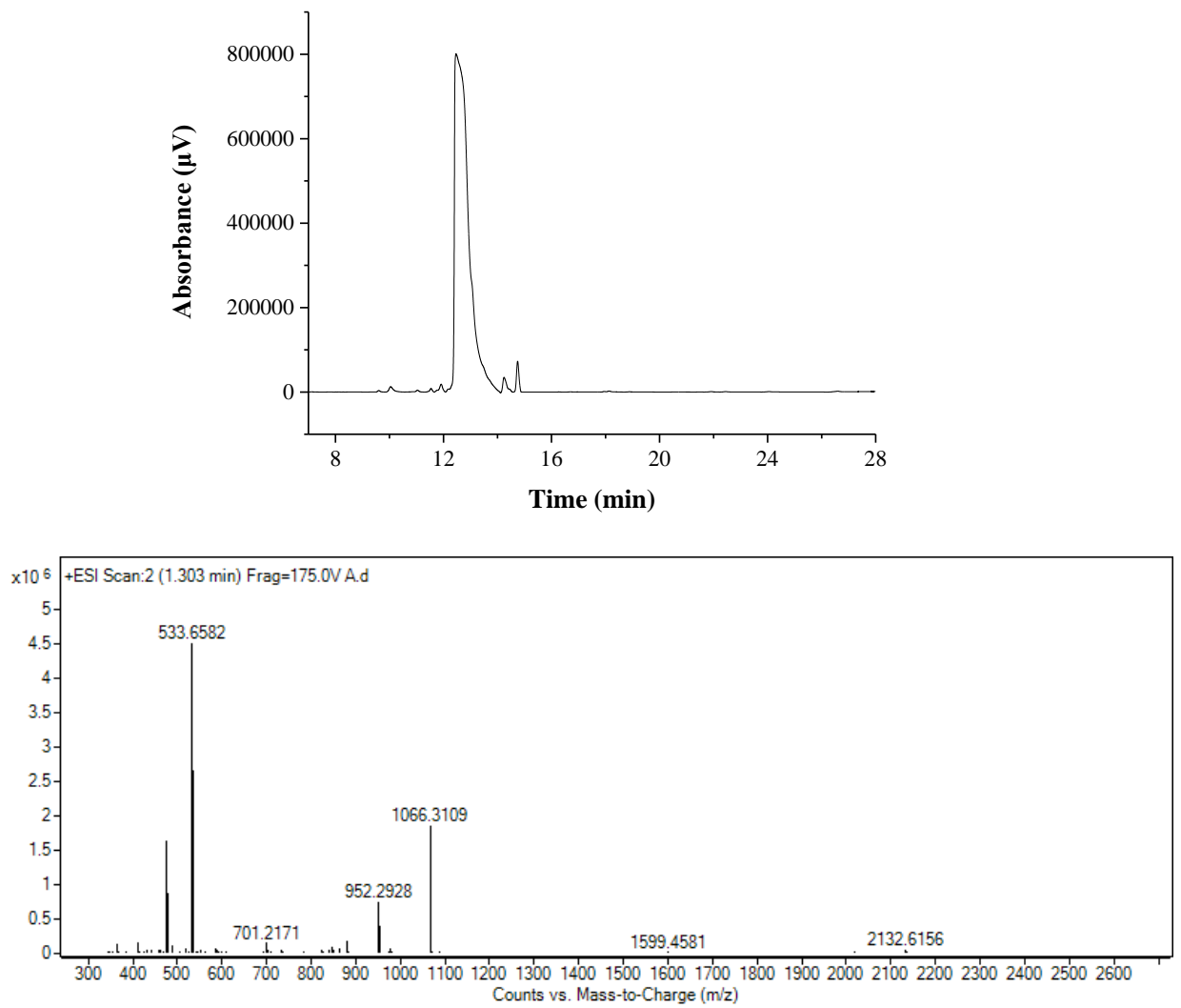

Peptide B-OBcM 

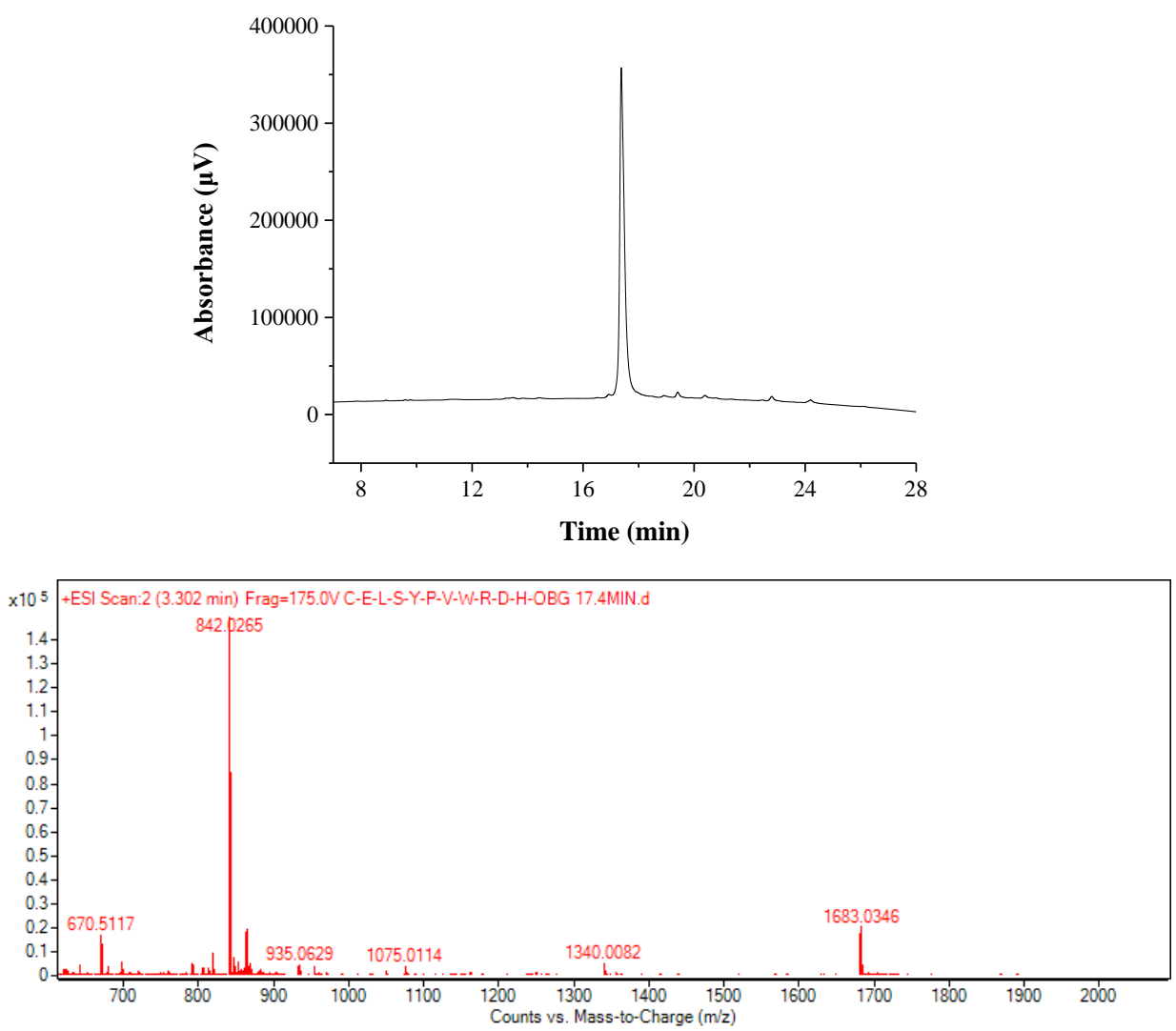

Peptide B-NHNH ${ }_{2}$
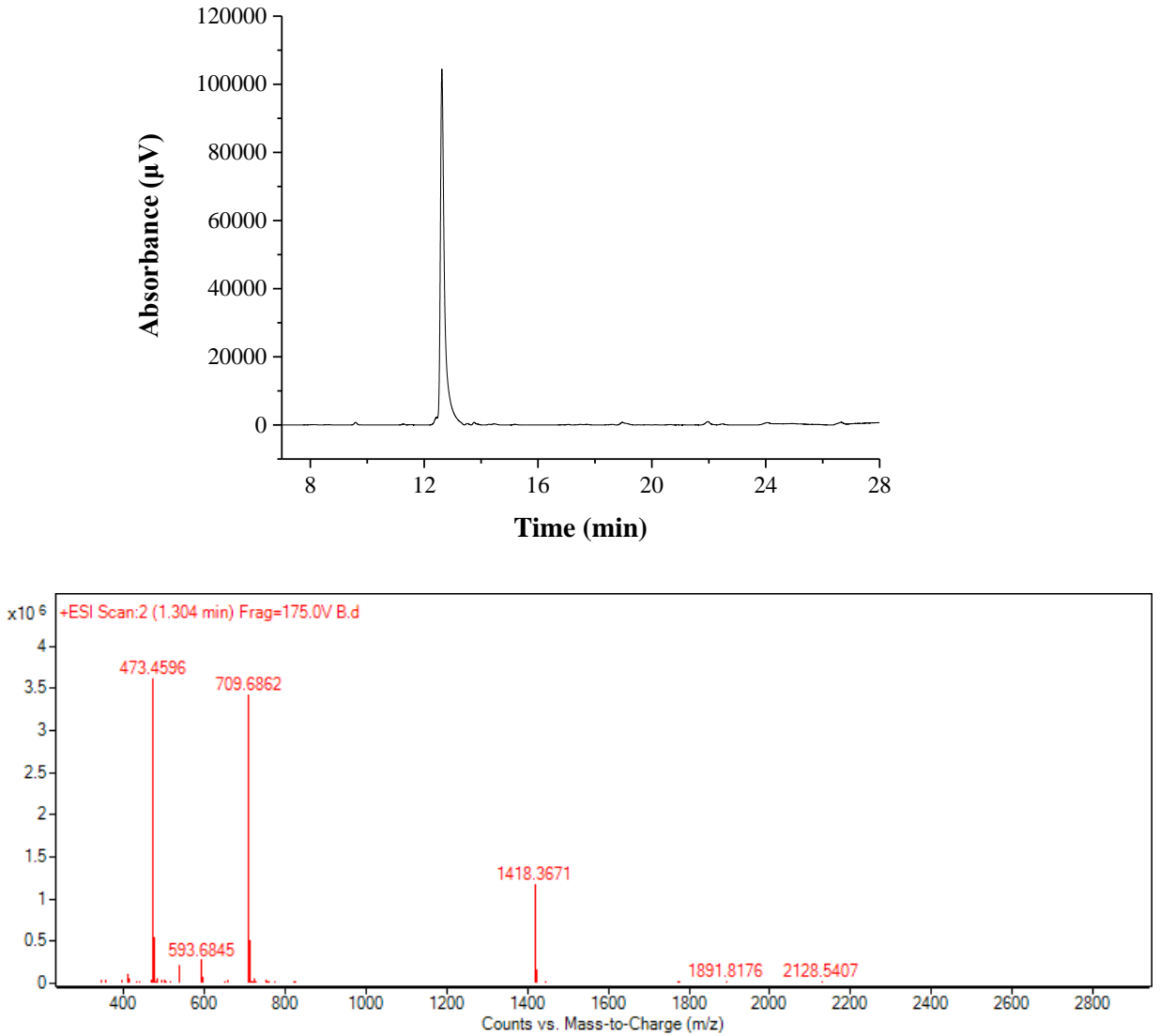
- Native Chemical Ligation
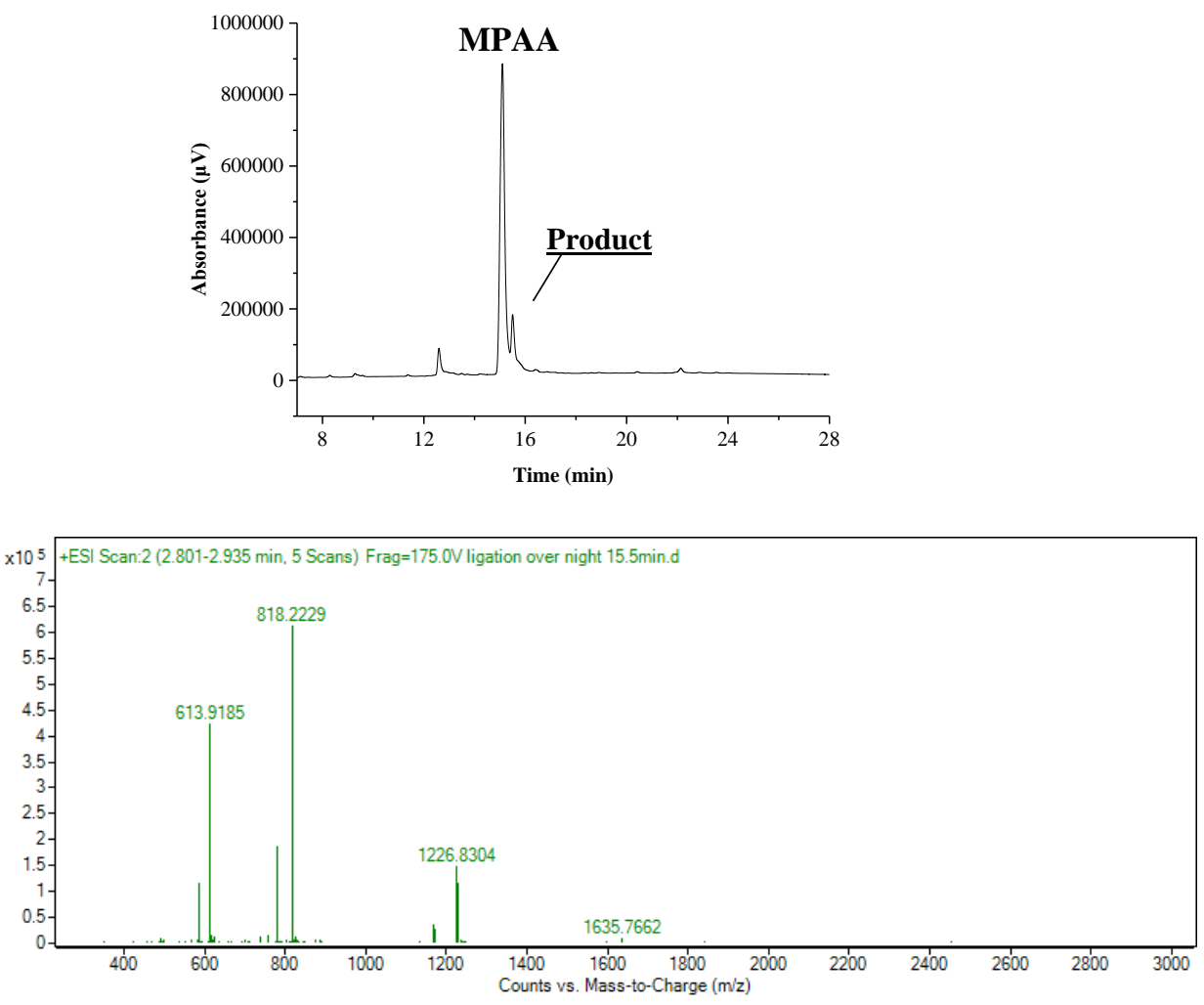


\begin{tabular}{|c|c|}
\hline Amino acids & Compatible side chain groups \\
\hline Tyrosine (Tyr) & $-\mathrm{O}^{t} \mathrm{Bu},-\mathrm{OH}^{*}$ \\
\hline Threonine (Thr) & $-\mathrm{OH}$ \\
\hline Serine (Ser) & $-\mathrm{OH}$ \\
\hline Glutamic acid (Glu) & $-\mathrm{CO}_{2}{ }^{t} \mathrm{Bu},-\mathrm{COOH}^{*}$ \\
\hline Aspartic acid (Asp) & $-\mathrm{CO}_{2}{ }^{t} \mathrm{Bu},-\mathrm{COOH}^{*}$ \\
\hline Asparagine (Asn) & $-\mathrm{CONHTrt},-\mathrm{CONH}_{2}{ }^{*}$ \\
\hline Glutamine (Gln) & $-\mathrm{CONHTrt},-\mathrm{CONH}_{2}{ }^{*}$ \\
\hline Cysteine (Cys) & $-\mathrm{STrt},-\mathrm{SH}^{*}$ \\
\hline Methionine (Met) & $-\mathrm{SMe}$ \\
\hline Histidine (His) & \\
\hline Arginine $(\mathrm{R})$ & \\
\hline Lysine (Lys) & -NHBoc \\
\hline Tryptophan (Trp) & \\
\hline
\end{tabular}

* Performed with PEG resin 
10. Copies of ${ }^{1} \mathrm{H}$ and ${ }^{13} \mathrm{C}$ NMR Spectra

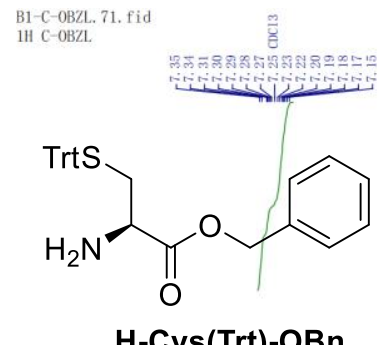

H-Cys(Trt)-OBn

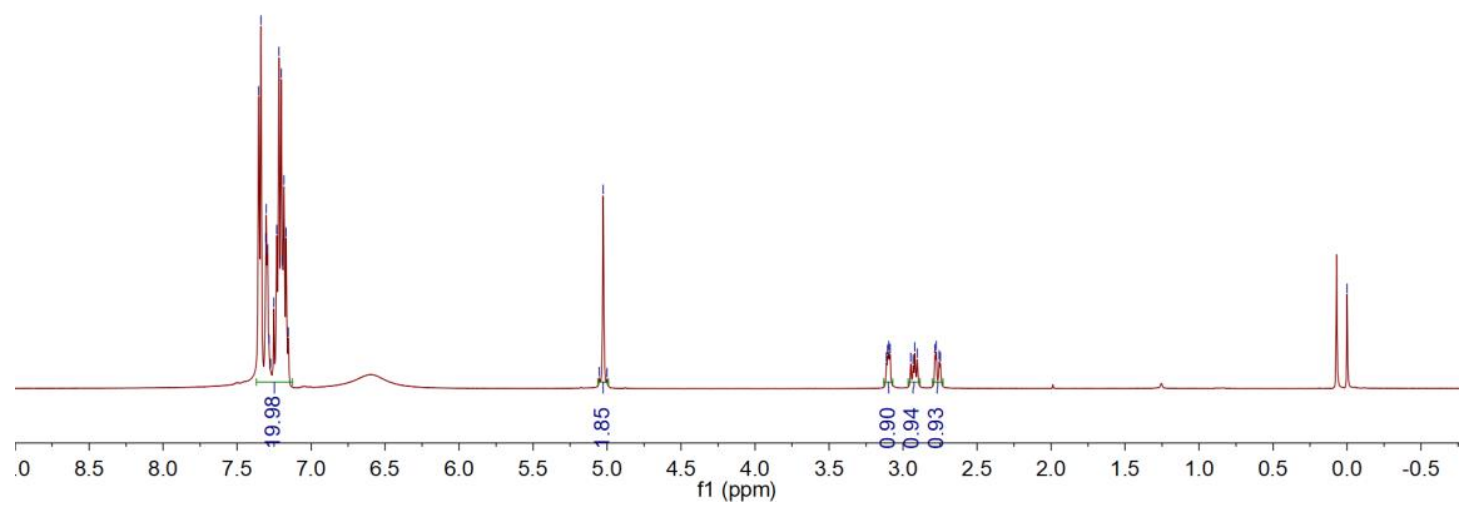

B1-C-0BZL. 73. fid

B1-C-OBZL
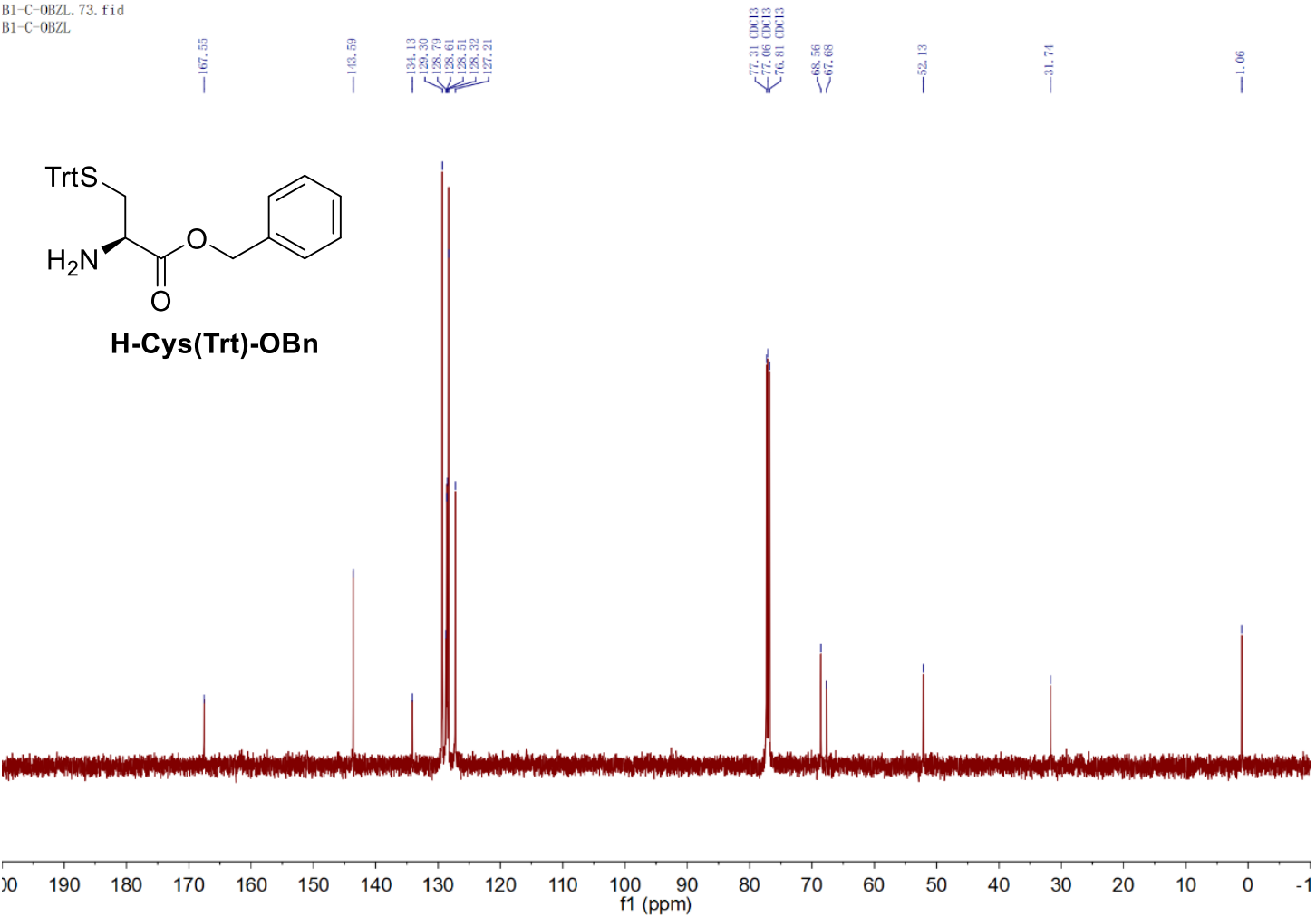


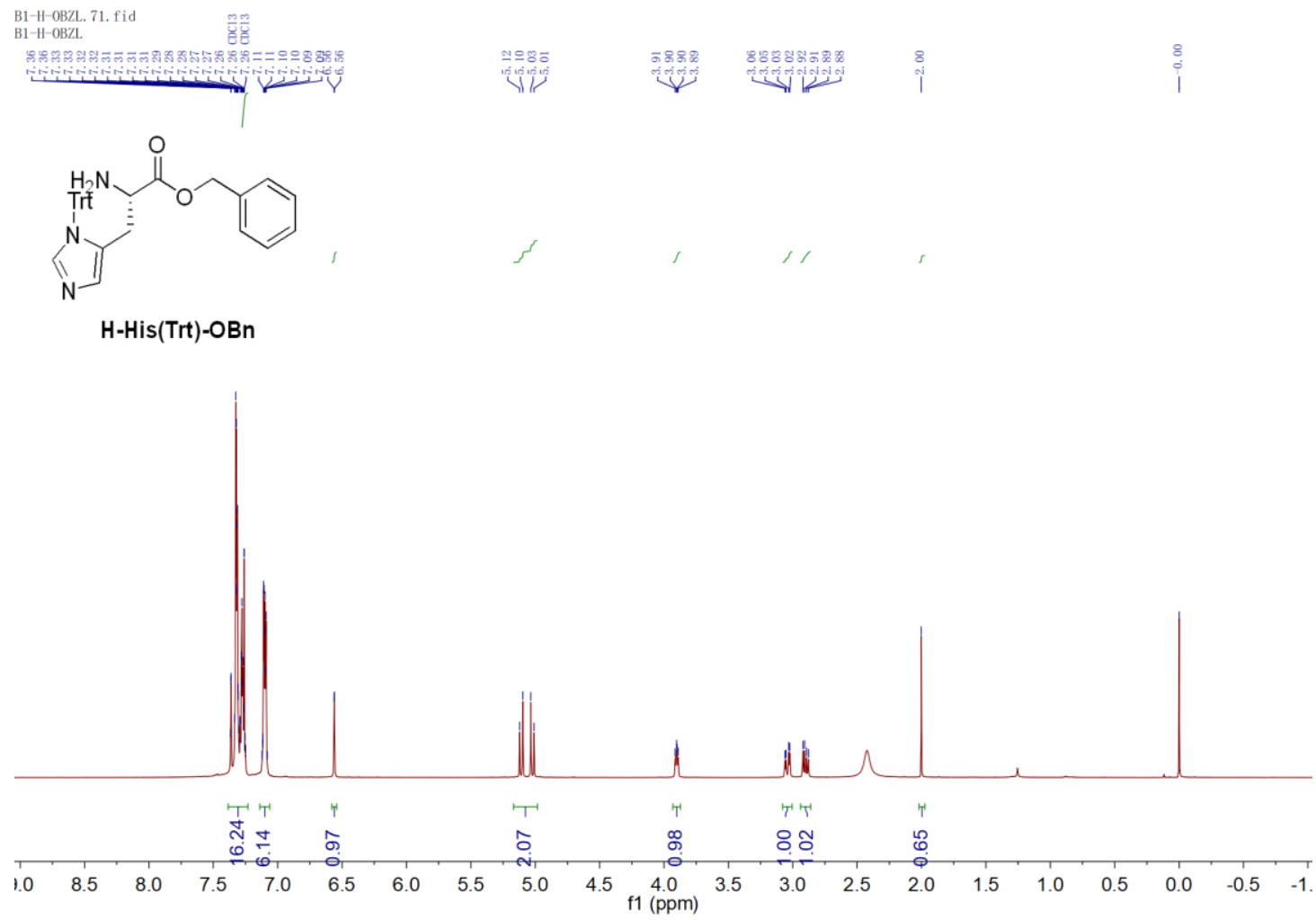

B1-H-OBZL. 73. fid

B1-H-0BZL

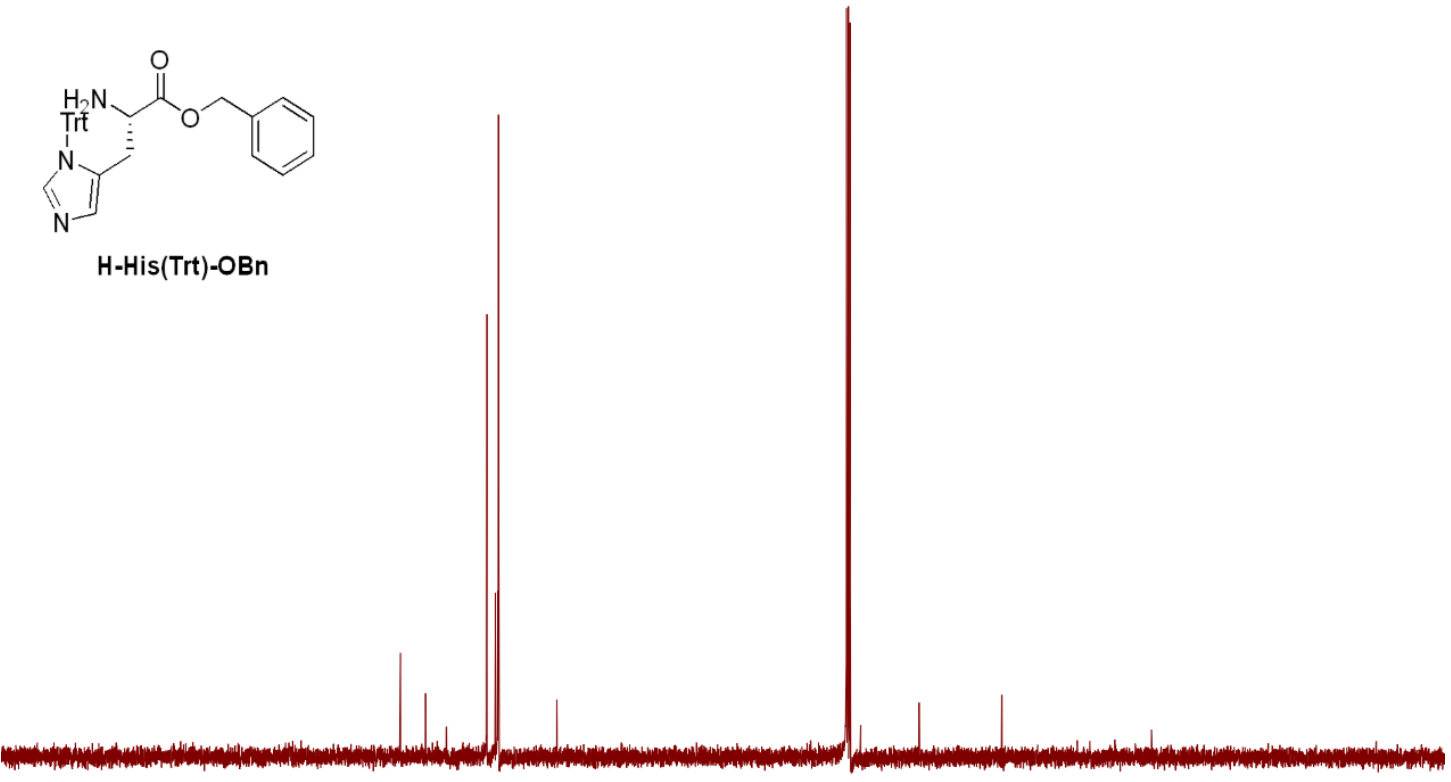

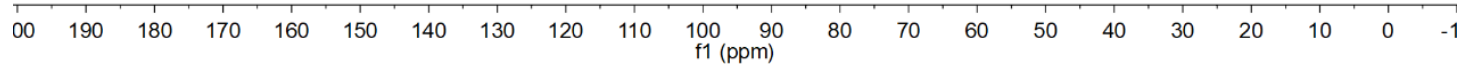




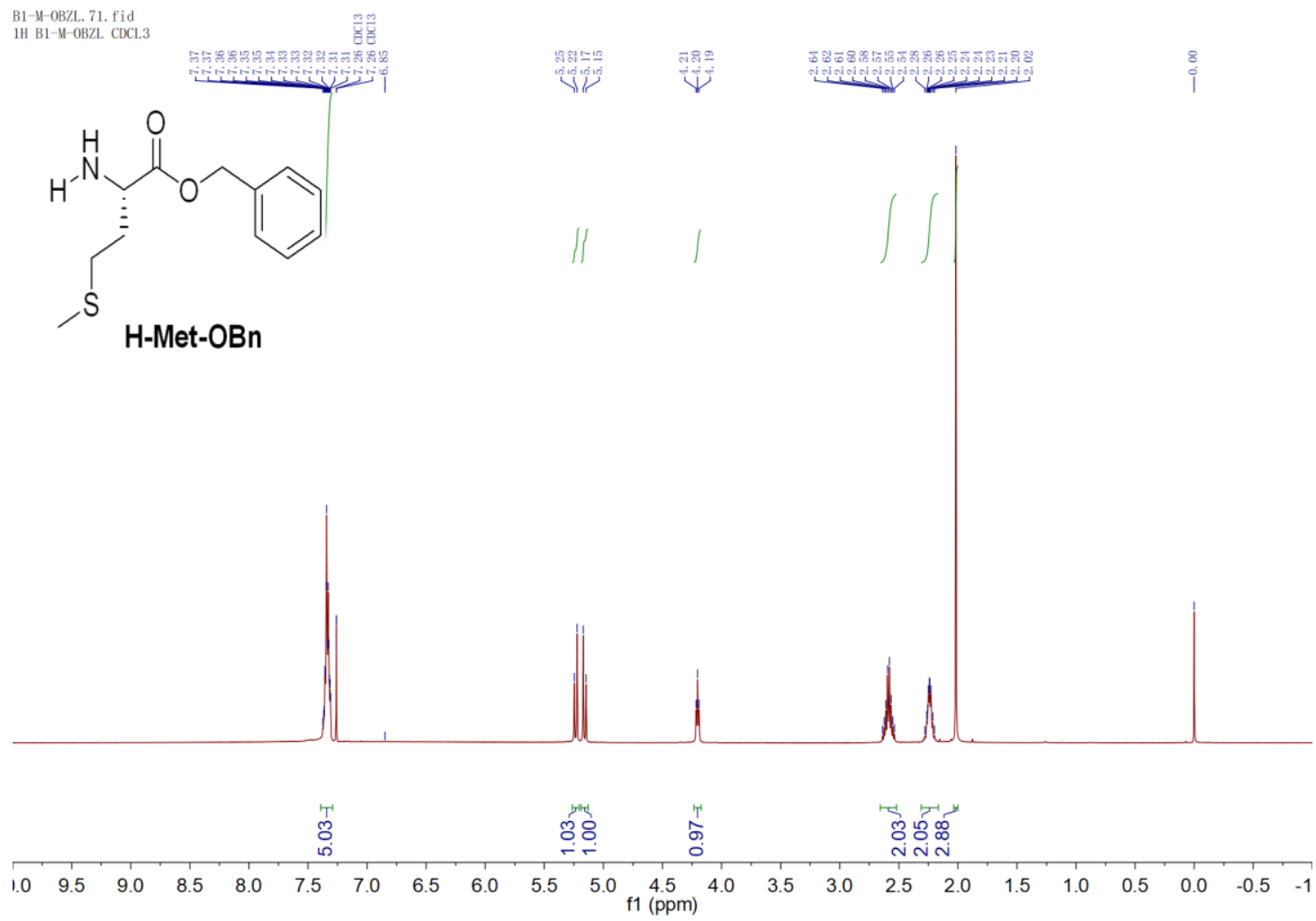

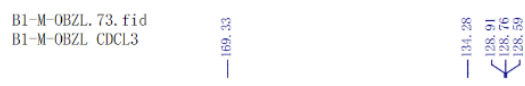<smiles>CSCC[C@H](NN)C(=O)OCc1ccccc1</smiles>

H-Met-OBn

$\begin{array}{lllllllllllllllllllll}00 & 190 & 180 & 170 & 160 & 150 & 140 & 130 & 120 & 110 & \begin{array}{l}100 \\ \mathrm{f} 1(\mathrm{ppm})\end{array} & 80 & 70 & 60 & 50 & 40 & 30 & 20 & 10 & 0\end{array}$ 
B1-N-0BZL. 71. fid
1H B1-N-0BZL. CDCL3

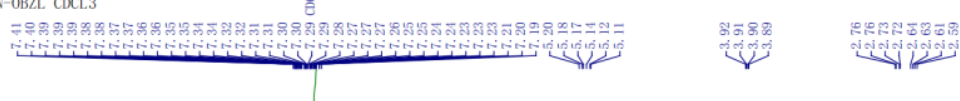<smiles>CCCNC(=O)C[C@H](N)C(=O)OCc1ccccc1</smiles>

H-Asn(Trt)-OBn

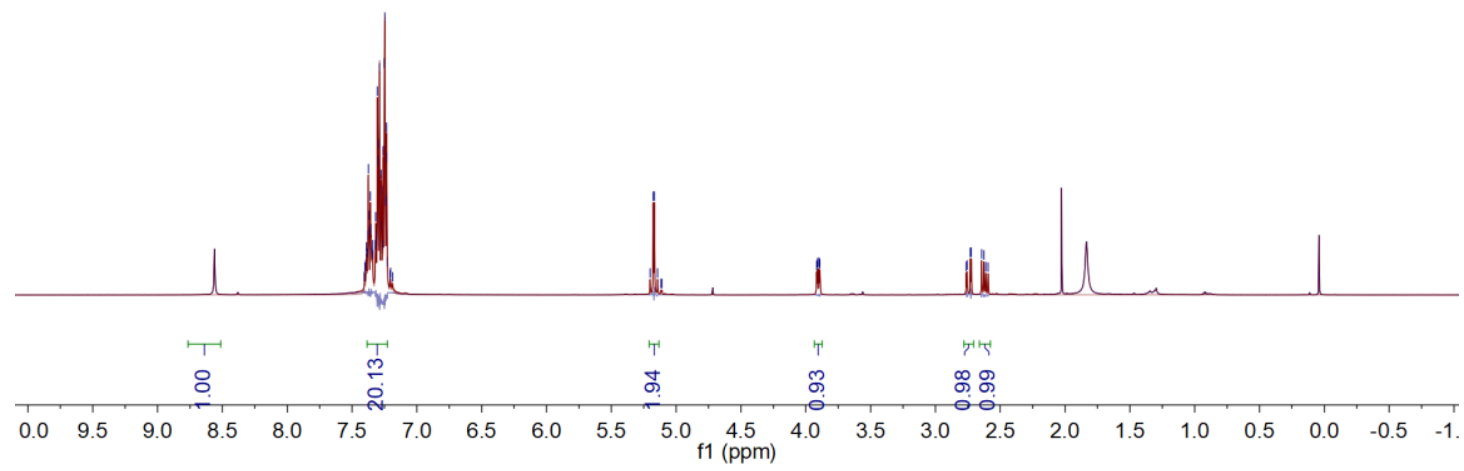
B1-N-OBZL. 73. fid
B1-N-OBZL<smiles>CCCNC(=O)CC(N)C(=O)OCc1ccccc1</smiles>

H-Asn(Trt)-OBn

$\begin{array}{llllllllllllllllllllllllll}230 & 220 & 210 & 200 & 190 & 180 & 170 & 160 & 150 & 140 & 130 & 120 & 110 & 100 & 90 & 80 & 70 & 60 & 50 & 40 & 30 & 20 & 10 & 0 & -10\end{array}$ 


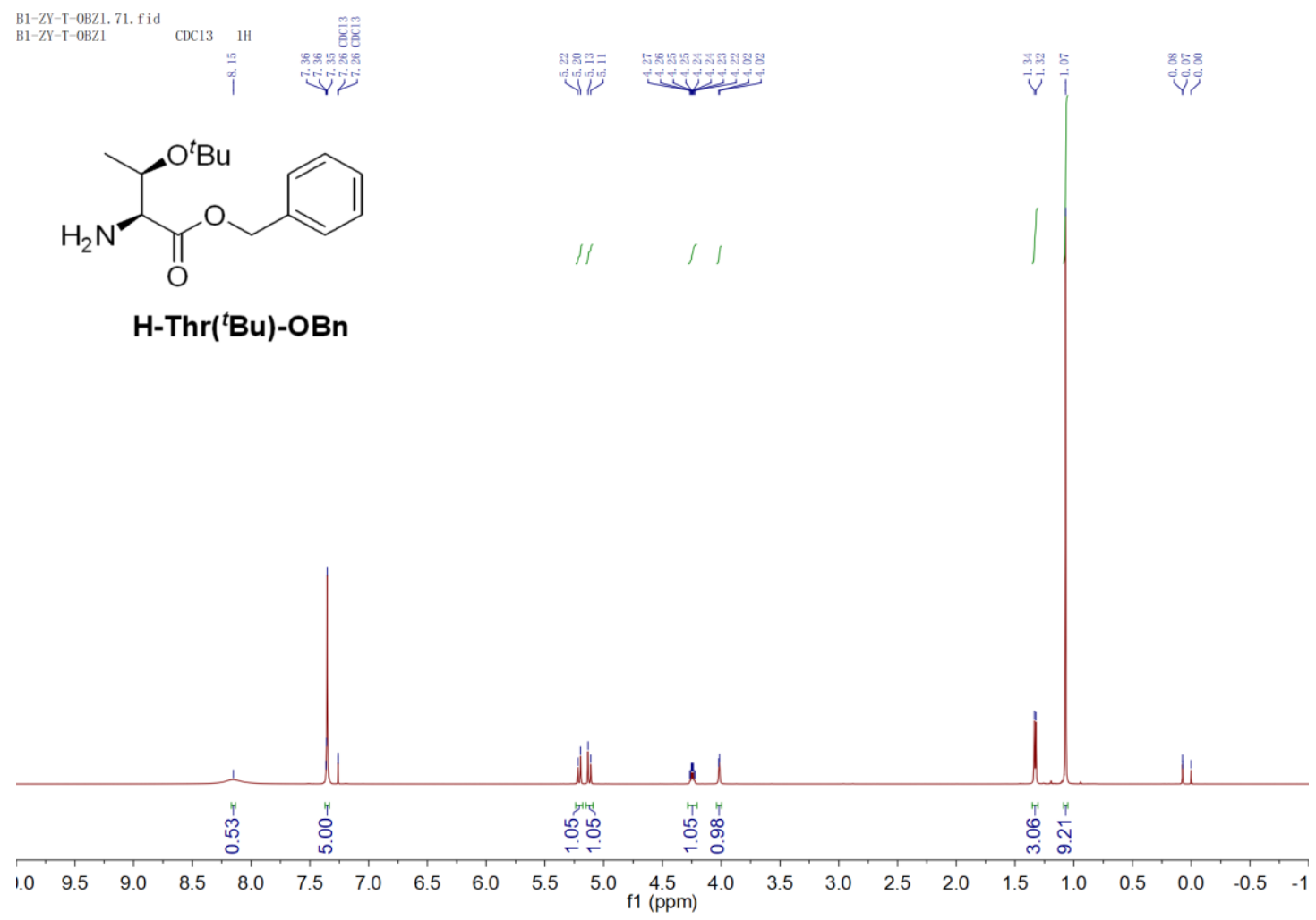

B1-T-OBZL. 74. fid
B1-T-0BZL.

(1)

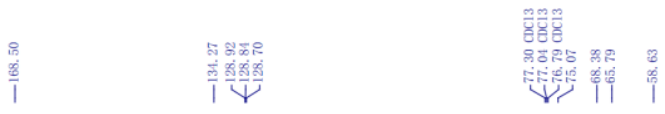

(O)

H-Thr( $\left.{ }^{t} \mathrm{Bu}\right)-\mathrm{OBn}$

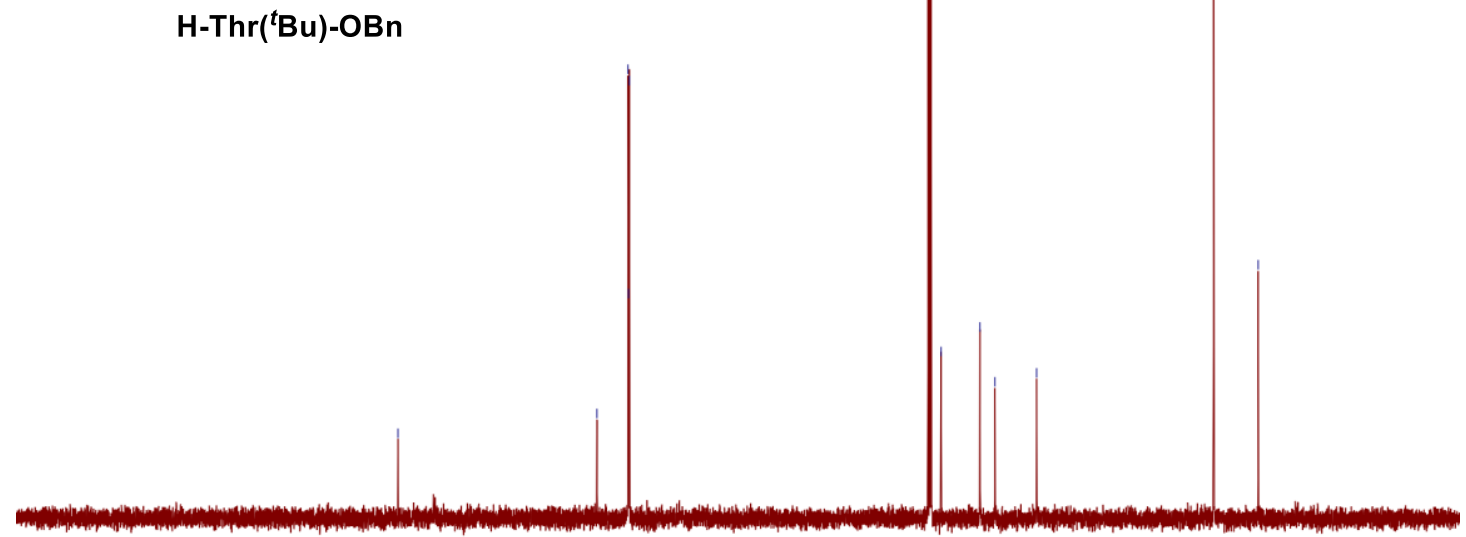

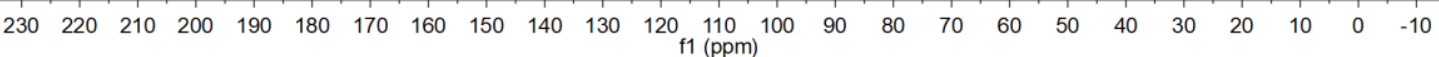




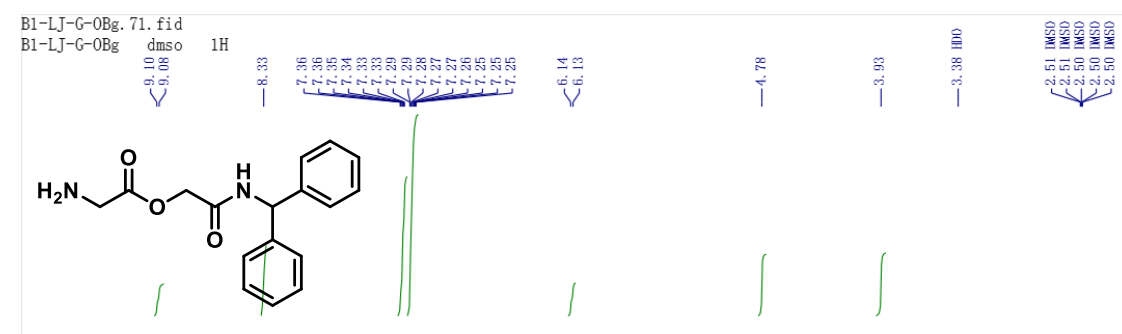

Compound H-Gly-OBcM

${ }^{1} \mathrm{H}_{\mathrm{NMR}}\left(\mathrm{DMSO}-\mathrm{d}_{6}, 500 \mathrm{MHz}\right)$

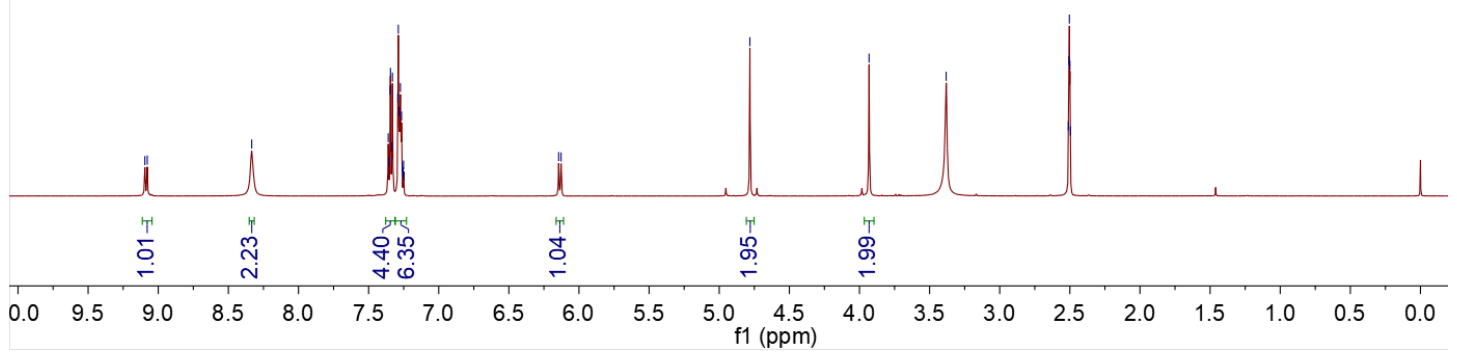

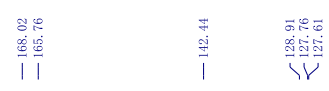

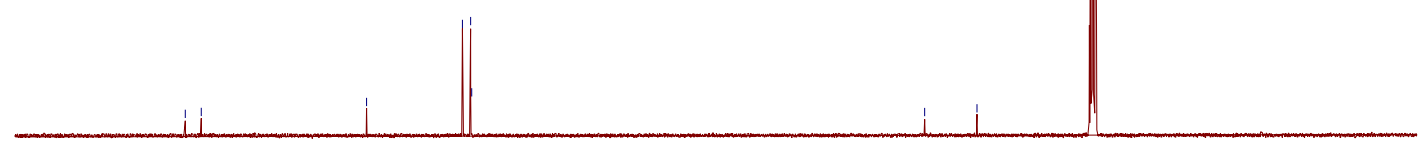

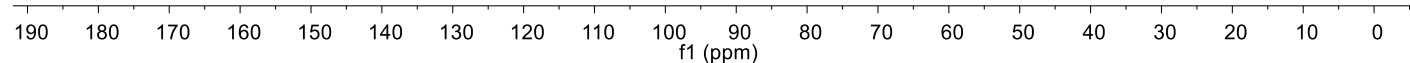



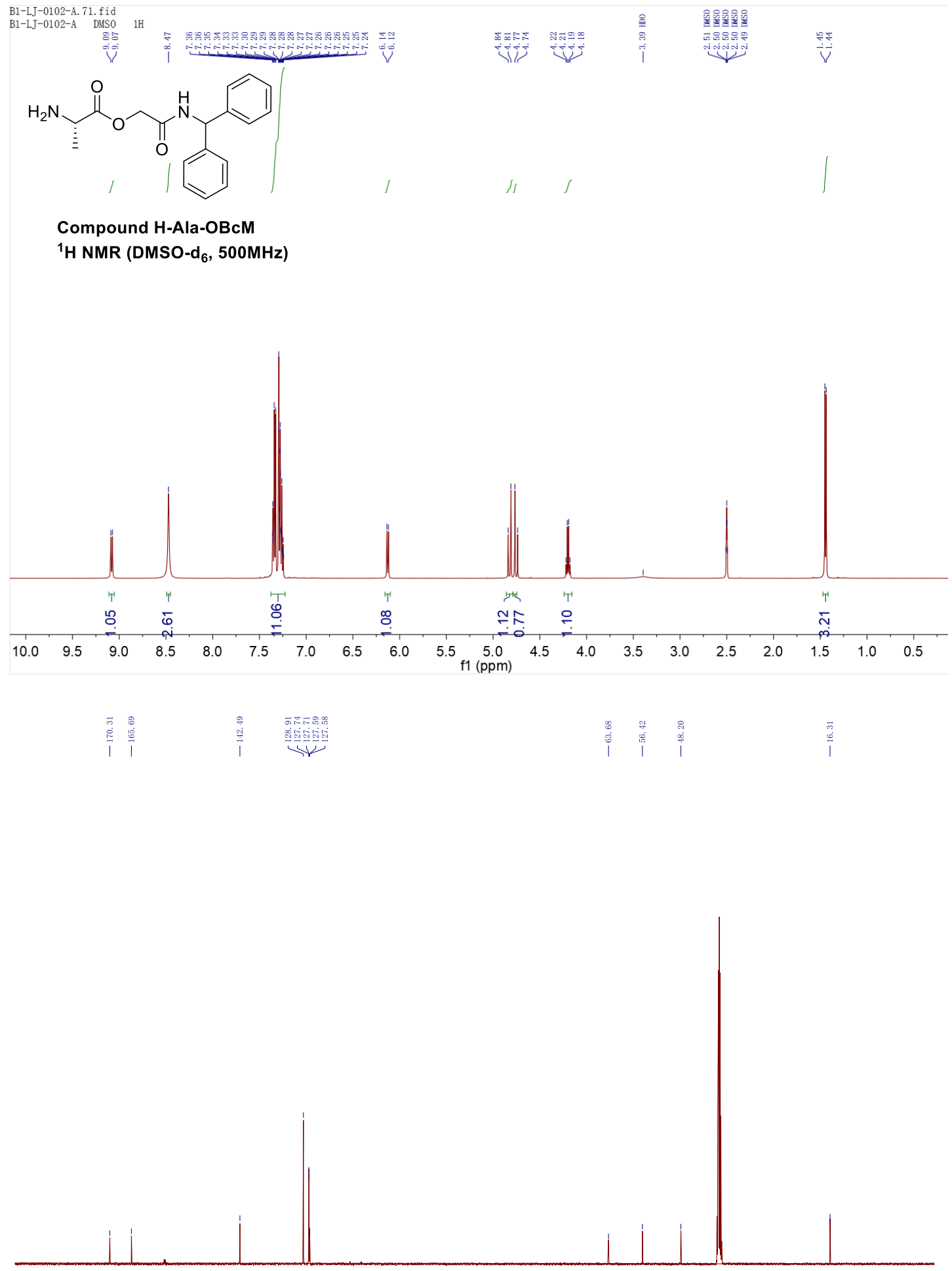

\begin{tabular}{lllllllllllllllllll}
\hline 90 & 180 & 170 & 160 & 150 & 140 & 130 & 120 & 110 & $100 \underset{\mathrm{f} 1(\mathrm{ppm})}{90}$ & 80 & 70 & 60 & 50 & 40 & 30 & 20 & 10 & 0
\end{tabular} 

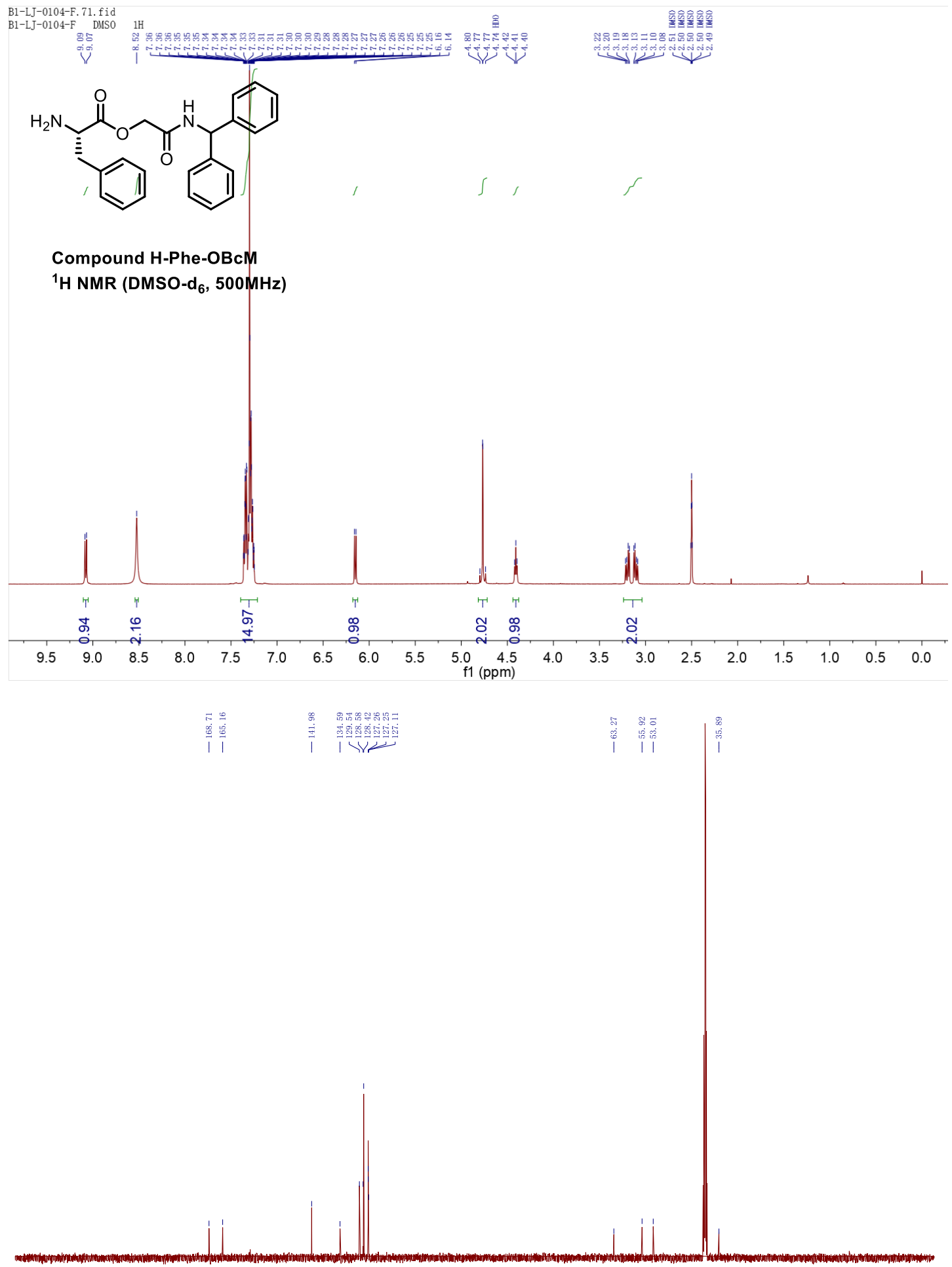

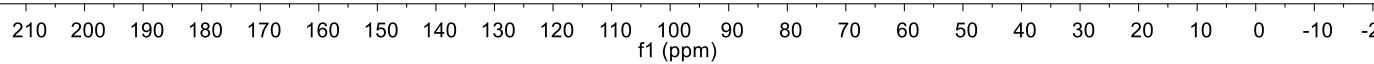




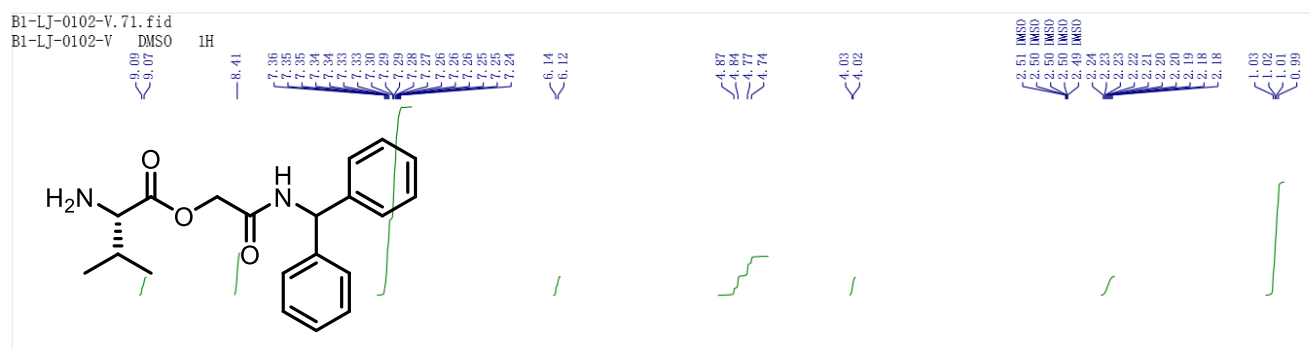

Compound H-Val-OBcM

${ }^{1} \mathrm{H} \mathrm{NMR}$ (DMSO-d $_{6}, 500 \mathrm{MHz}$ )
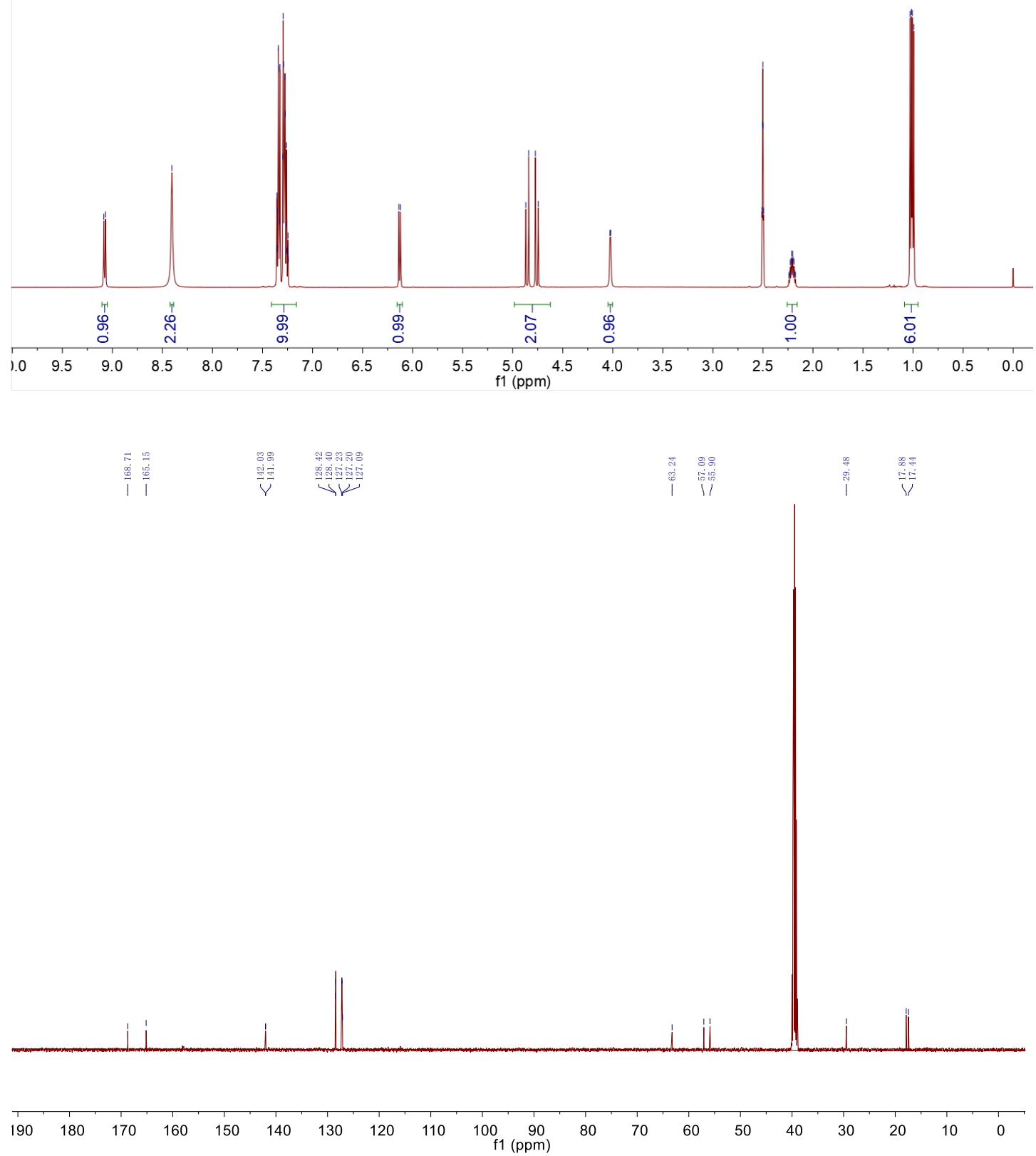


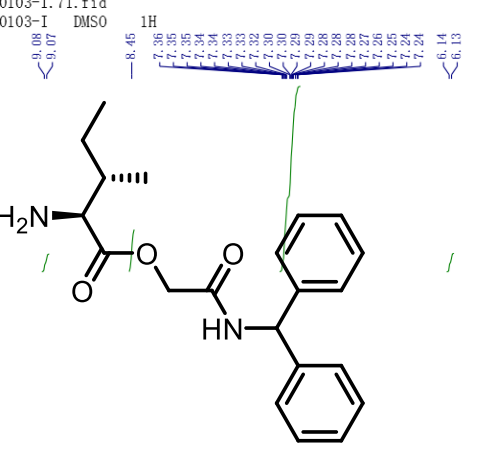

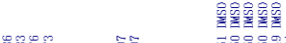

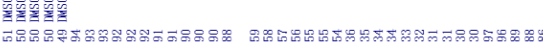

H-Ile-OBcM

${ }^{1} \mathrm{H}_{\mathrm{NMR}}$ (DMSO-d, $500 \mathrm{MHz}$ )
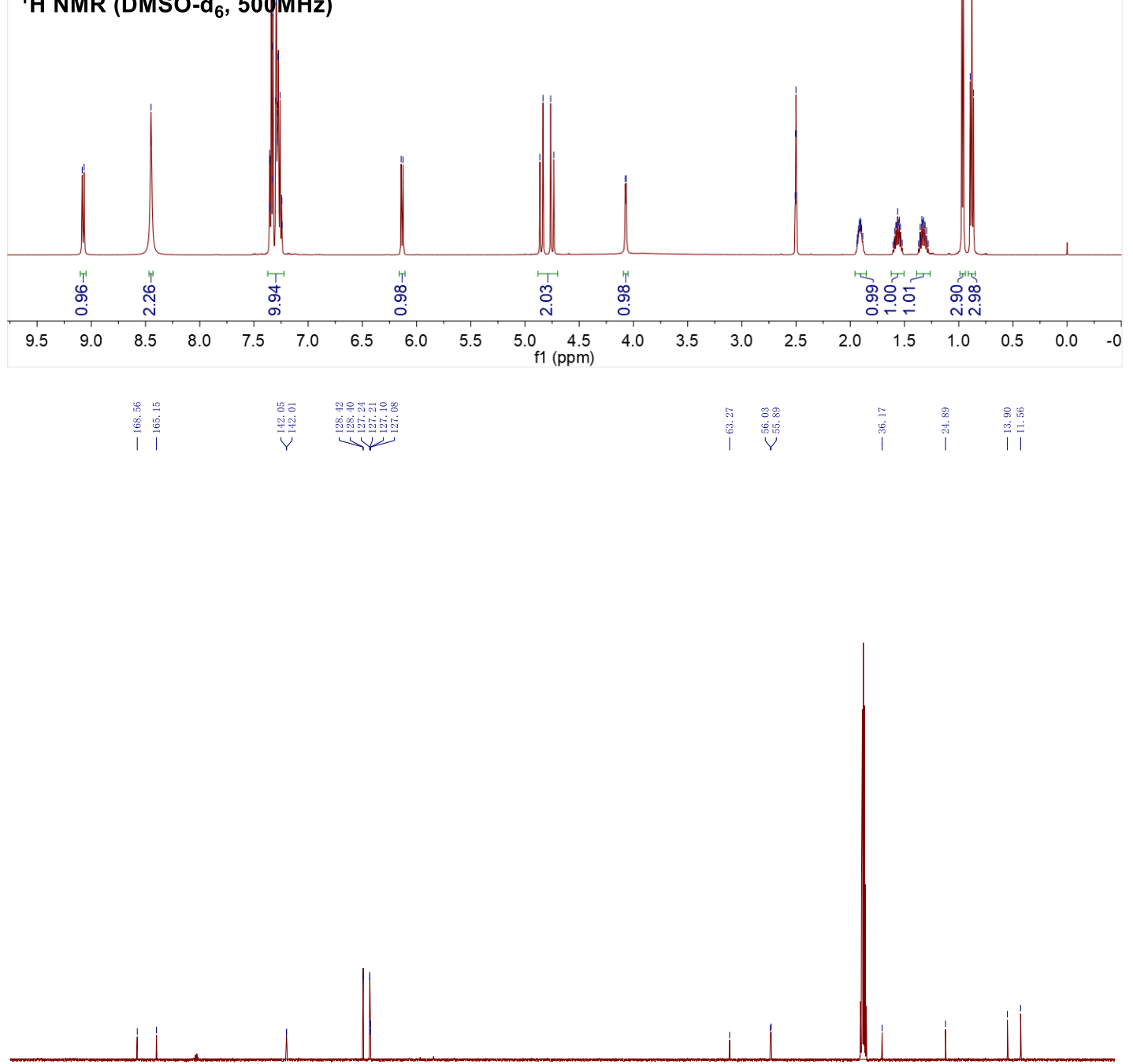

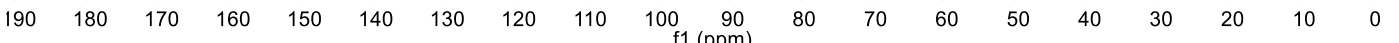



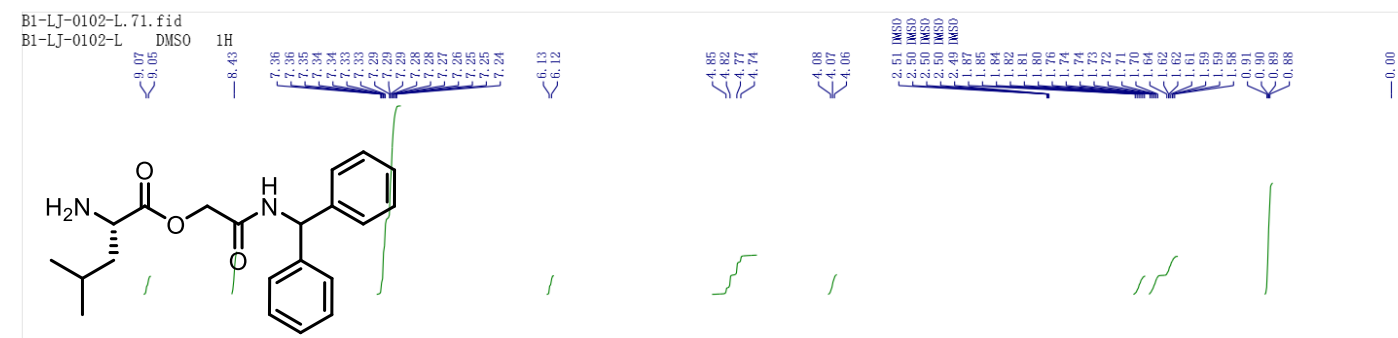

Compound H-Leu-OBcM

${ }^{1} \mathrm{H}$ NMR (DMSO-d 6 , 500MHz)

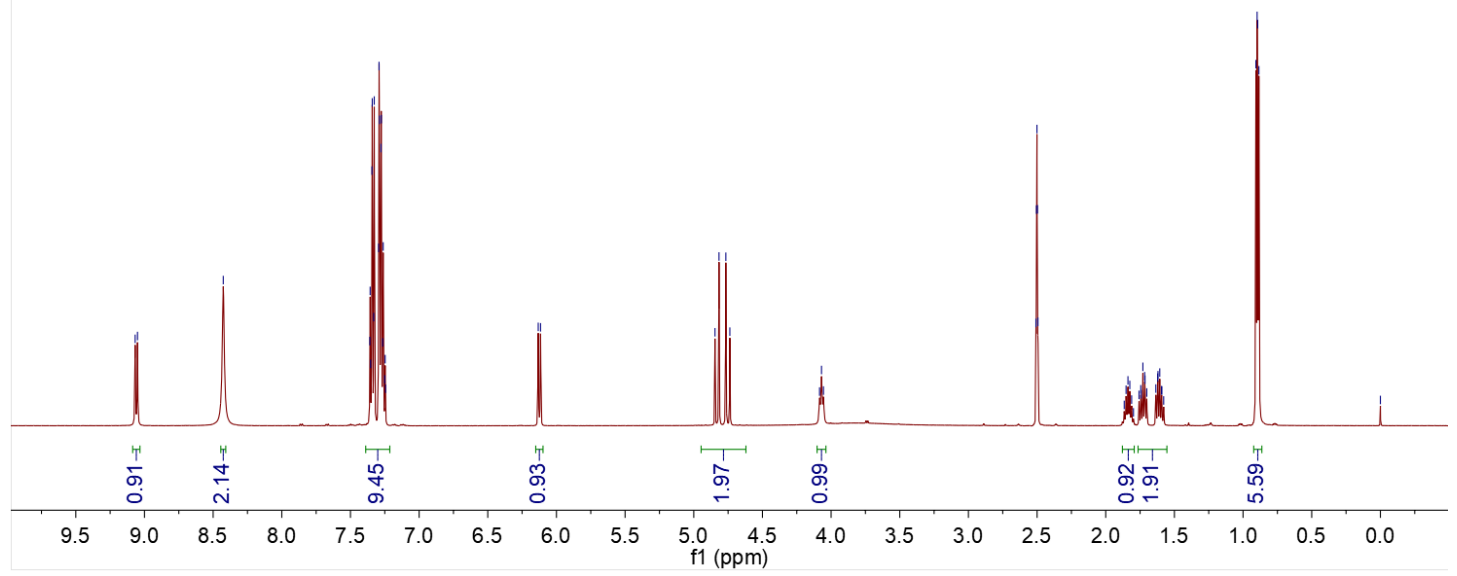

\begin{tabular}{|c|c|}
\hline $\mathbb{N}$ & bi \\
\hline & 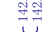 \\
\hline
\end{tabular}

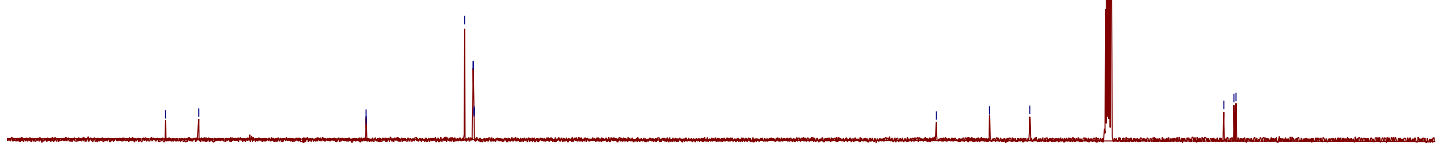

$\begin{array}{rrrrrrrrrr}190 & 180 & 170 & 160 & 150 & 140 & 130 & 120 & 110 & \begin{array}{r}100 \\ \mathrm{f} 1(\mathrm{ppm})\end{array}\end{array}$

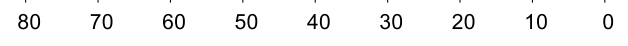




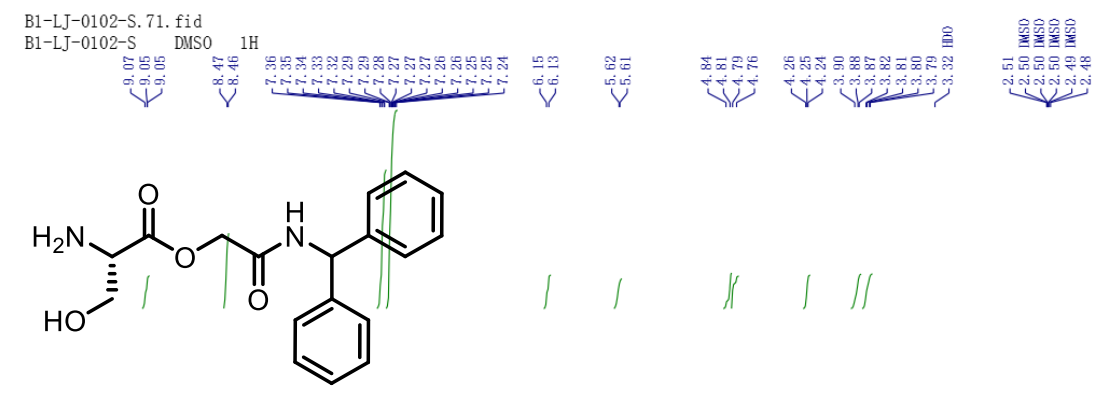

Compound H-Ser-OBcM

${ }^{1} \mathrm{H}_{\mathrm{NMR}}\left(\mathrm{DMSO}_{-1}, \mathrm{~d}_{6}, 500 \mathrm{MHz}\right)$

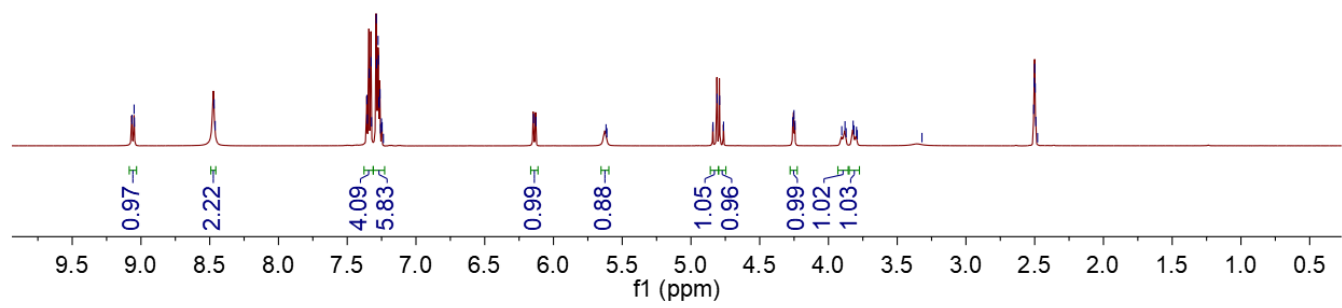

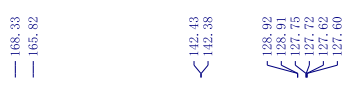

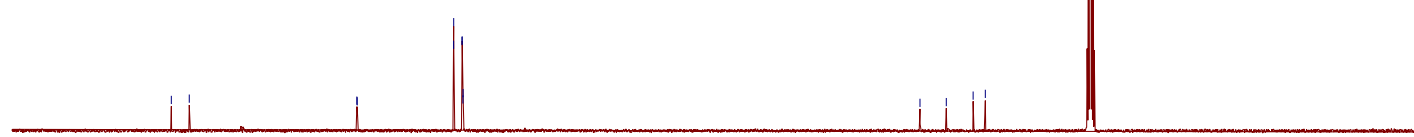

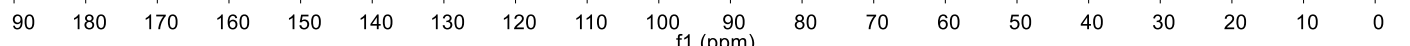




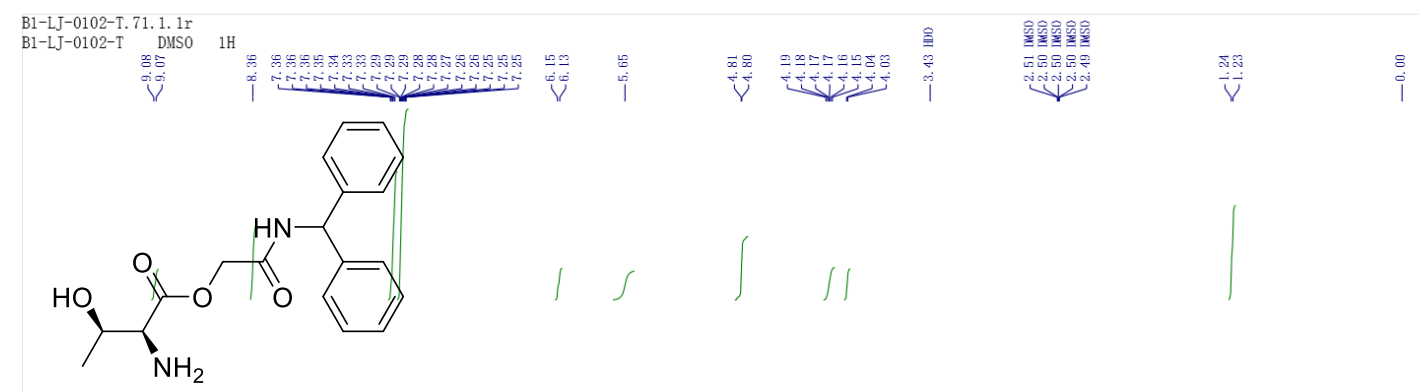

Compound H-Thr-OBcM

${ }^{1} \mathrm{H}$ NMR (DMSO-d $6,500 \mathrm{MHz}$ )
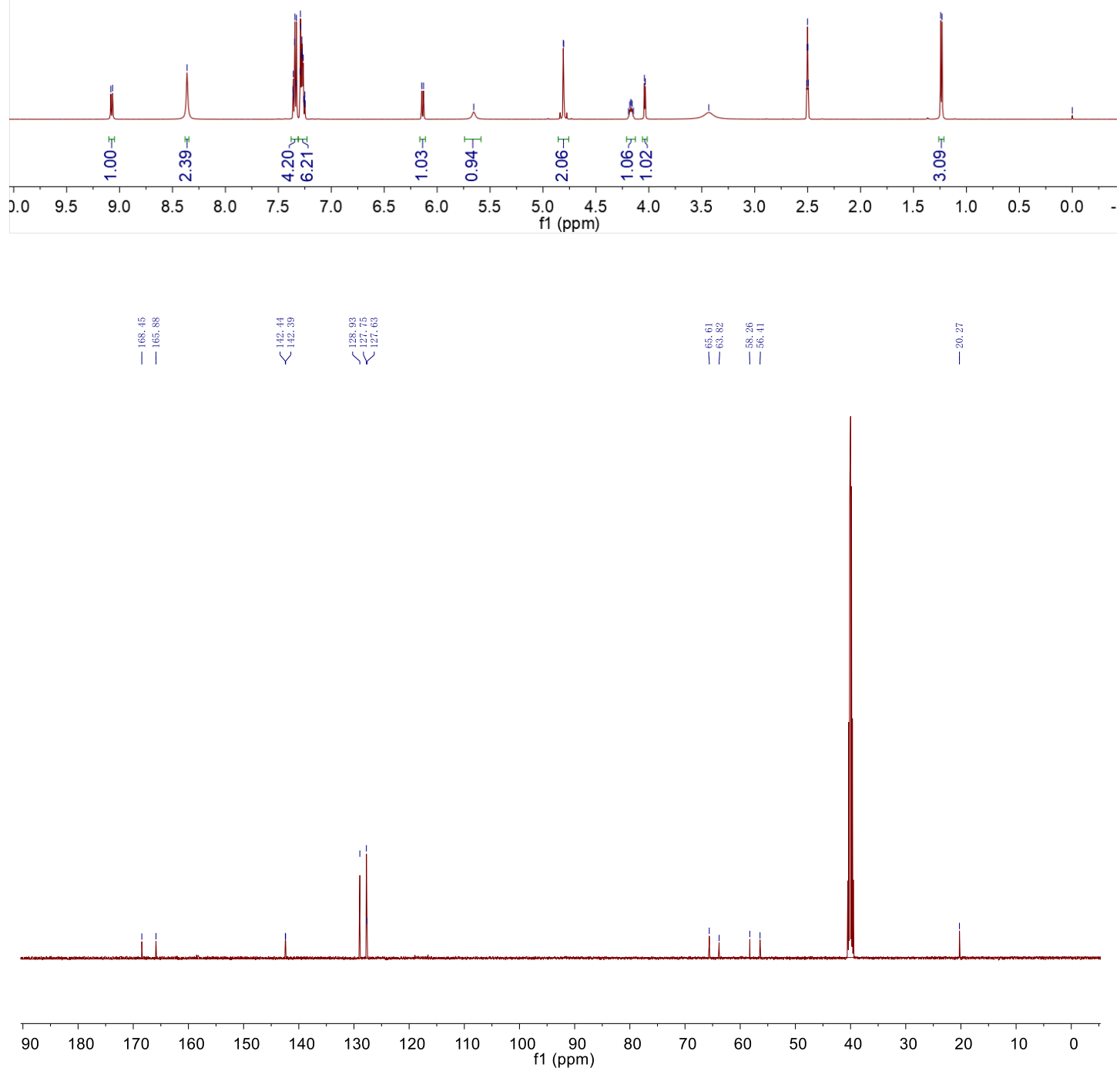


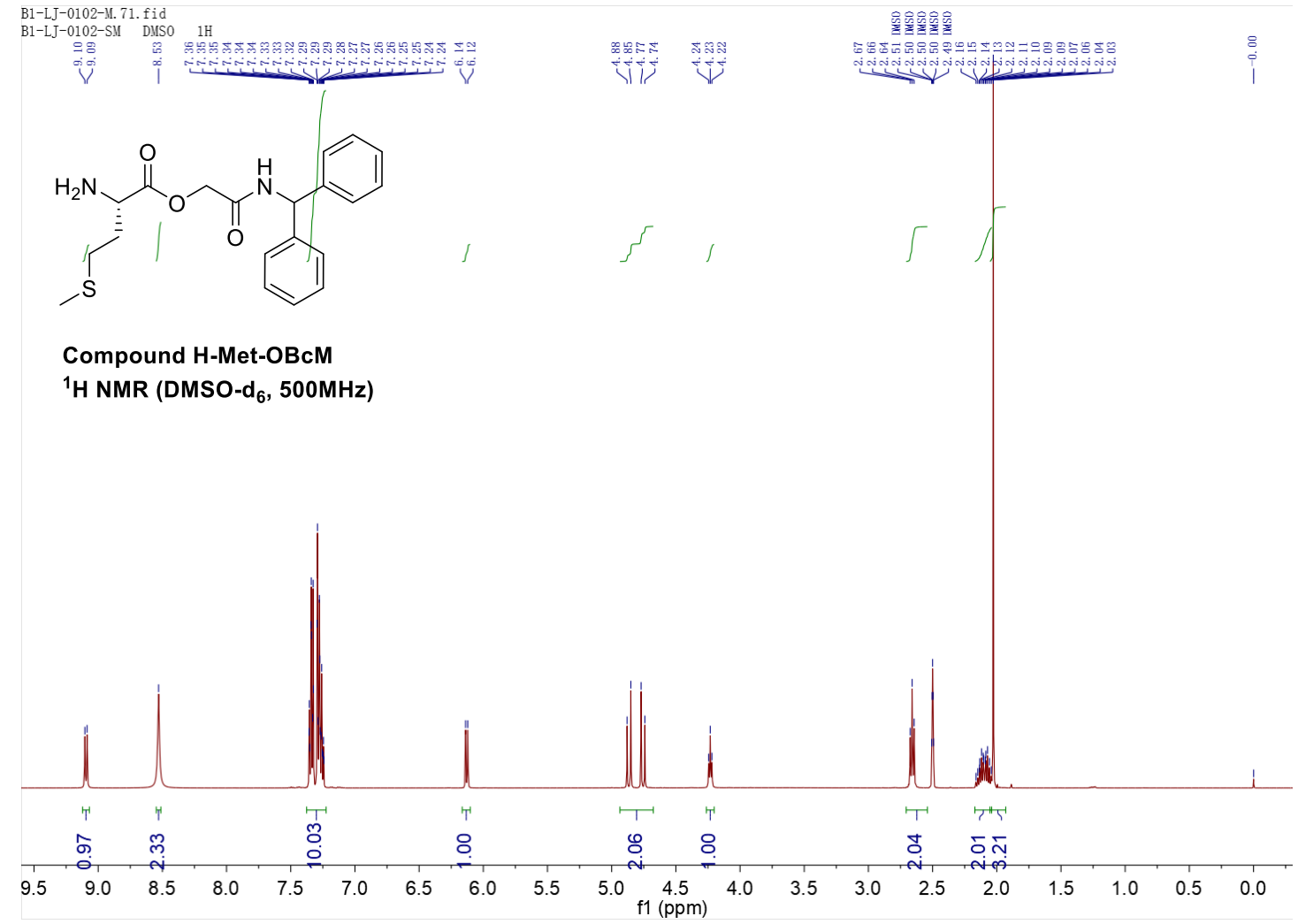

\begin{tabular}{|c|c|c|c|c|c|c|}
\hline 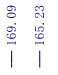 & 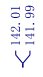 & 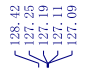 & $\begin{array}{l}\text { \% } \\
\text { \% } \\
1\end{array}$ & 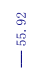 & $\begin{array}{l}2 \\
2 \\
0 \\
0 \\
0\end{array}$ & 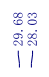 \\
\hline
\end{tabular}

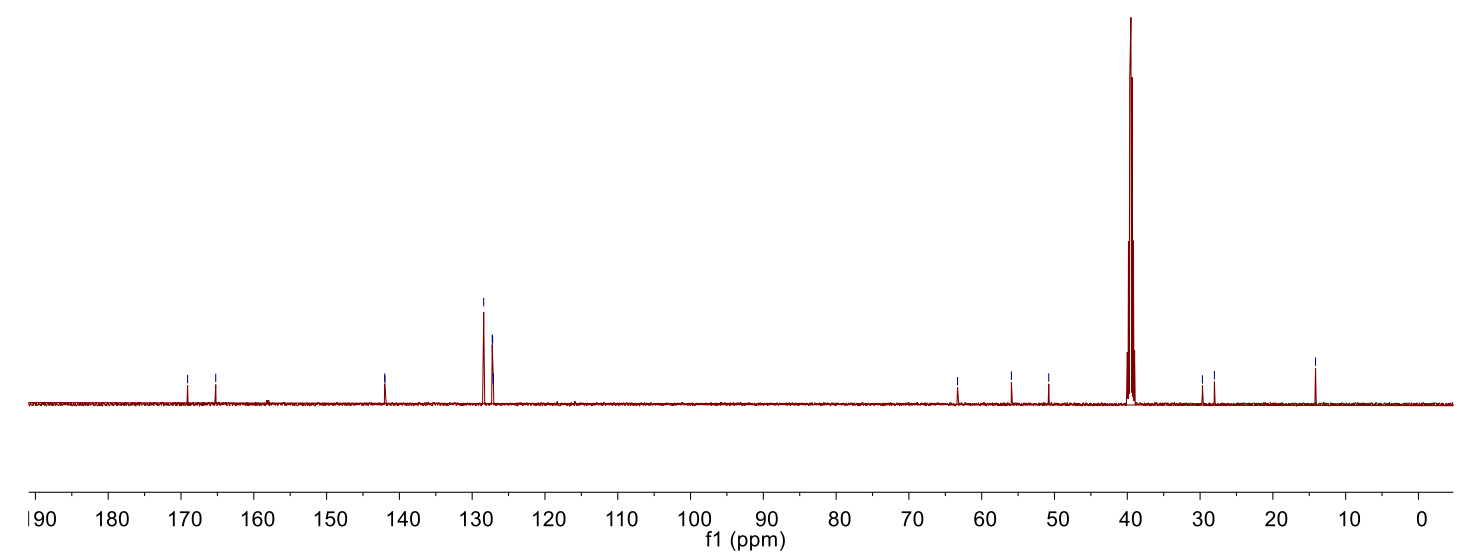



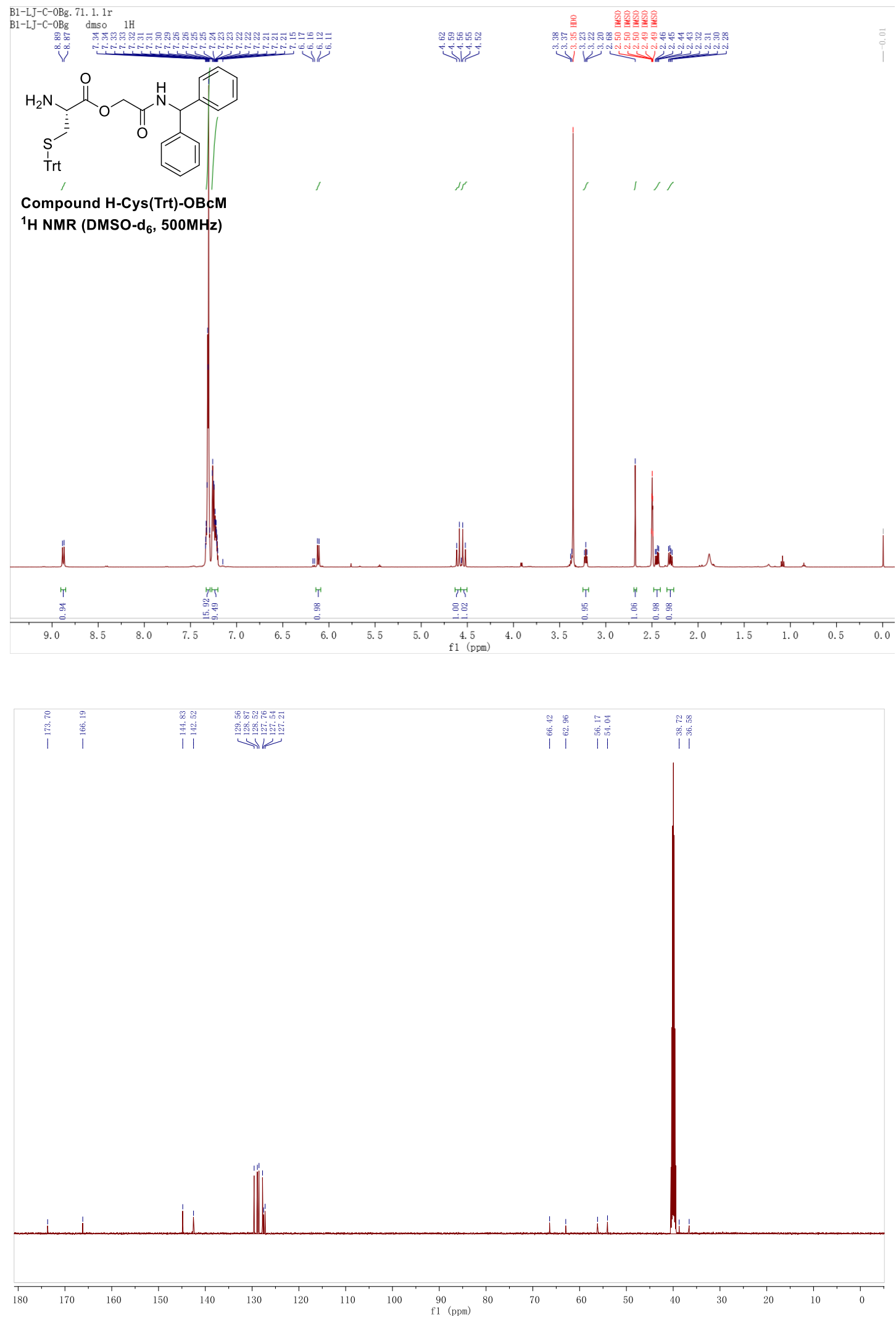

S56 


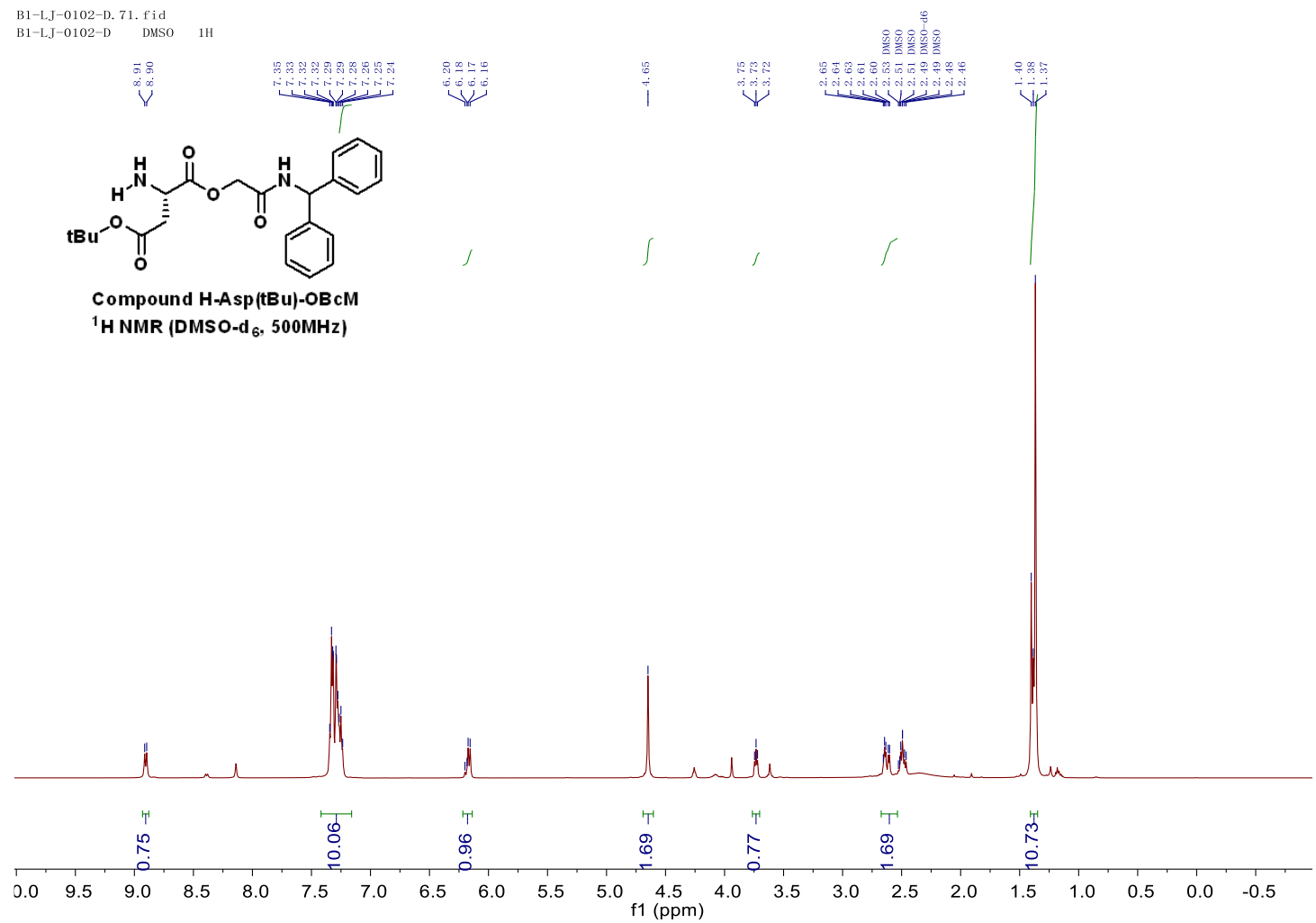

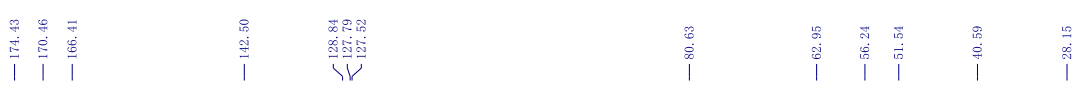

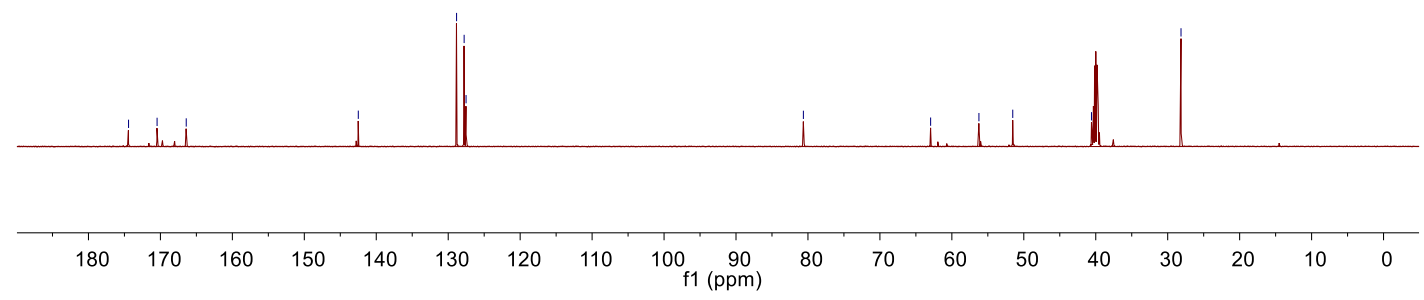




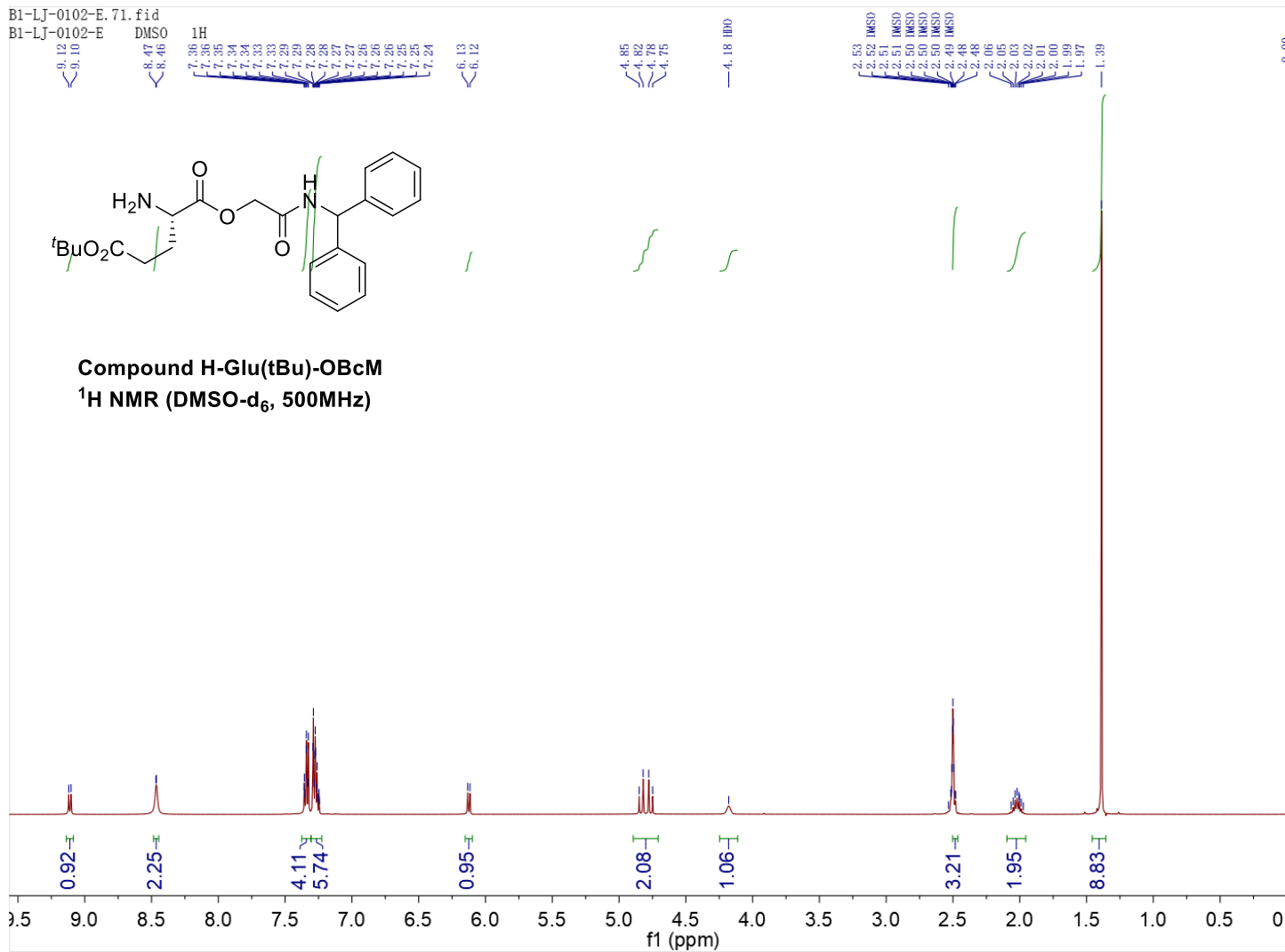

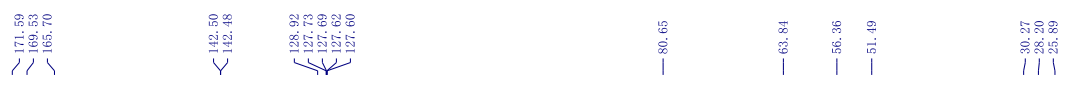

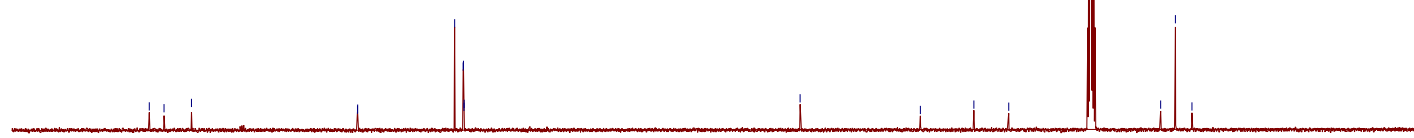

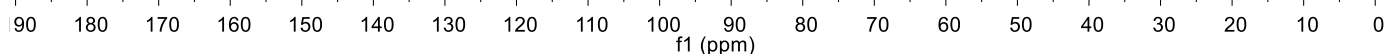



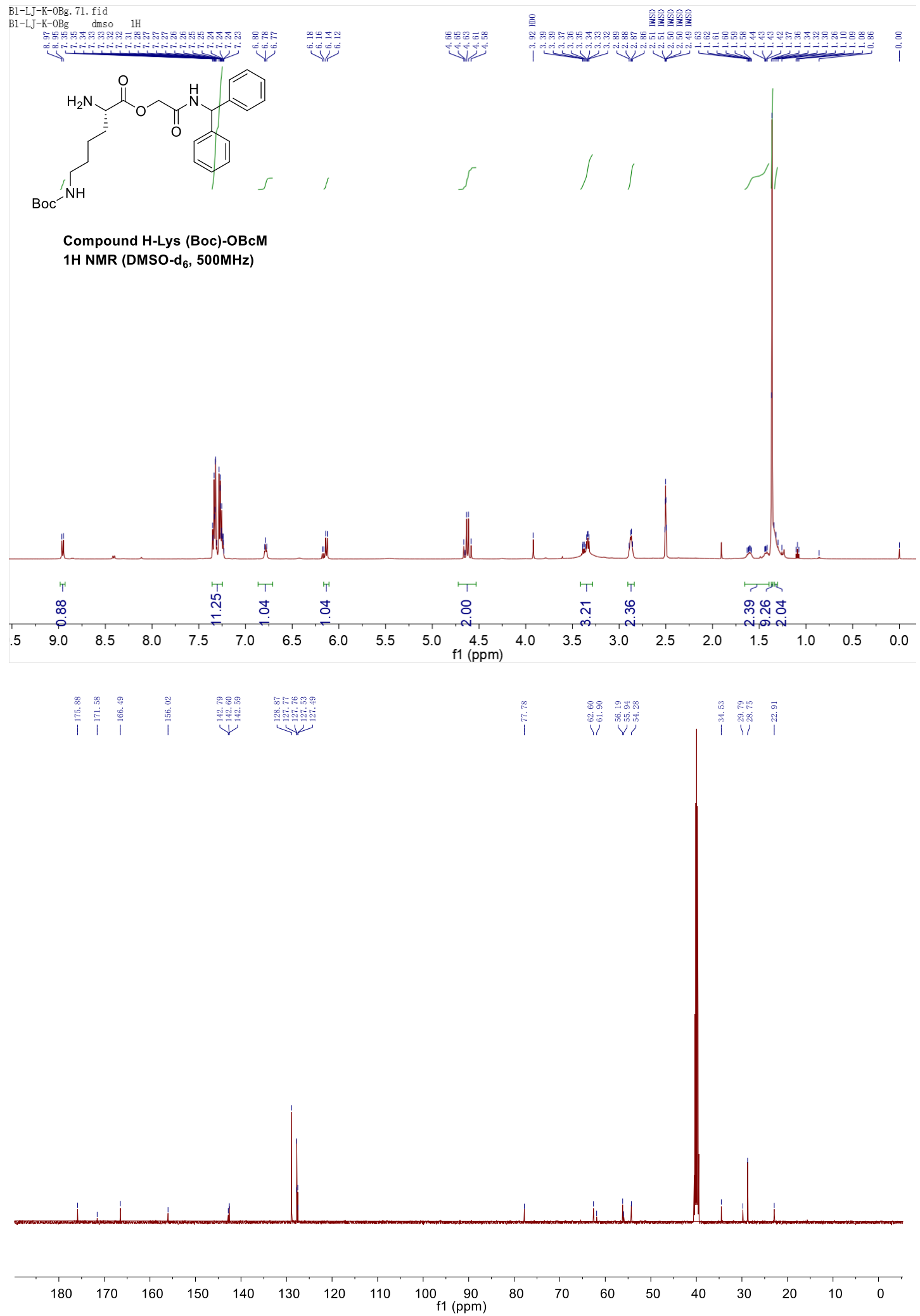


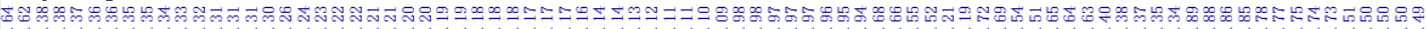
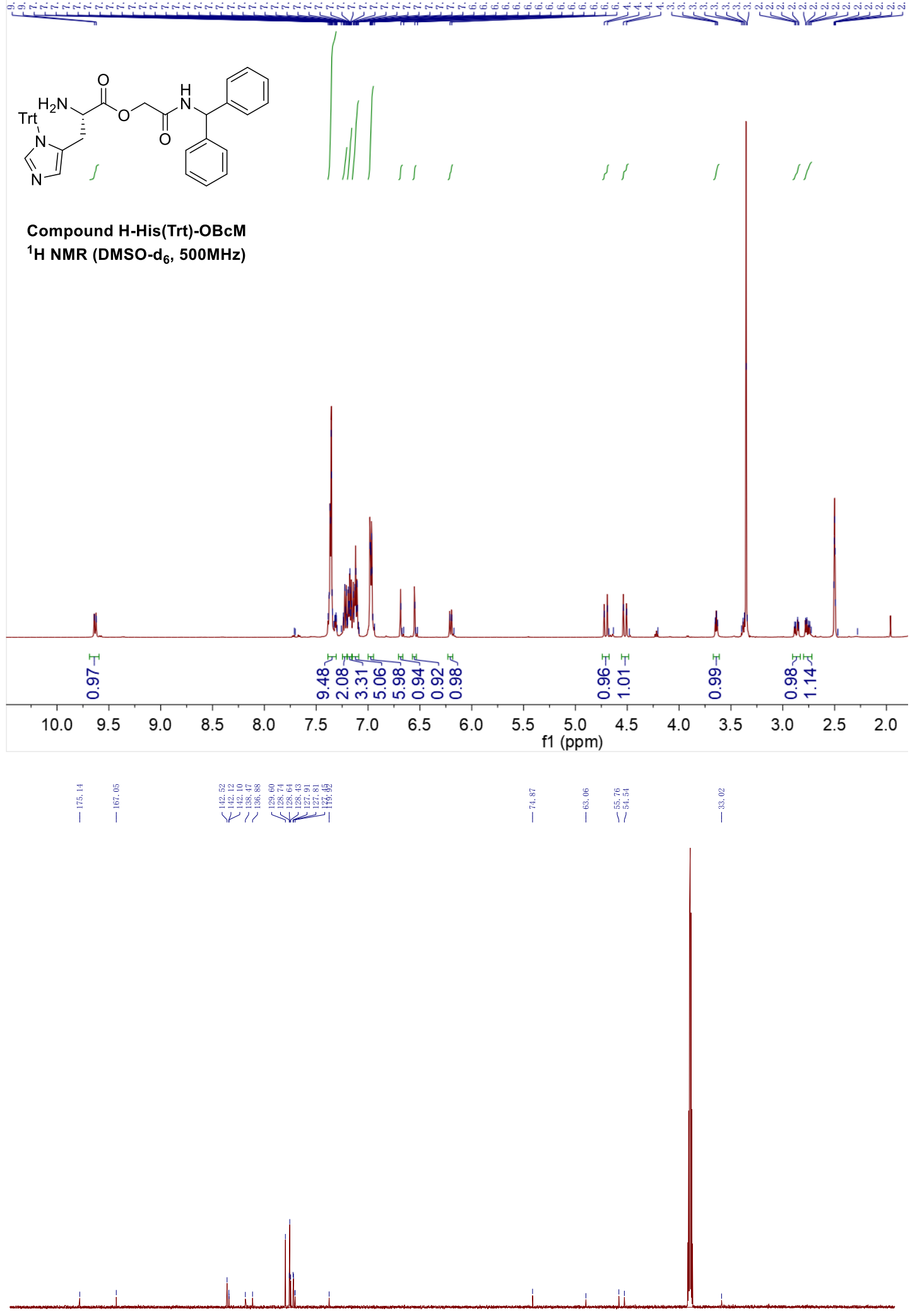

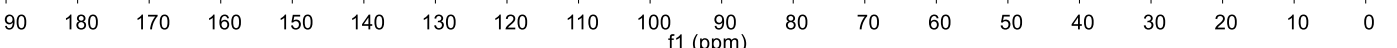




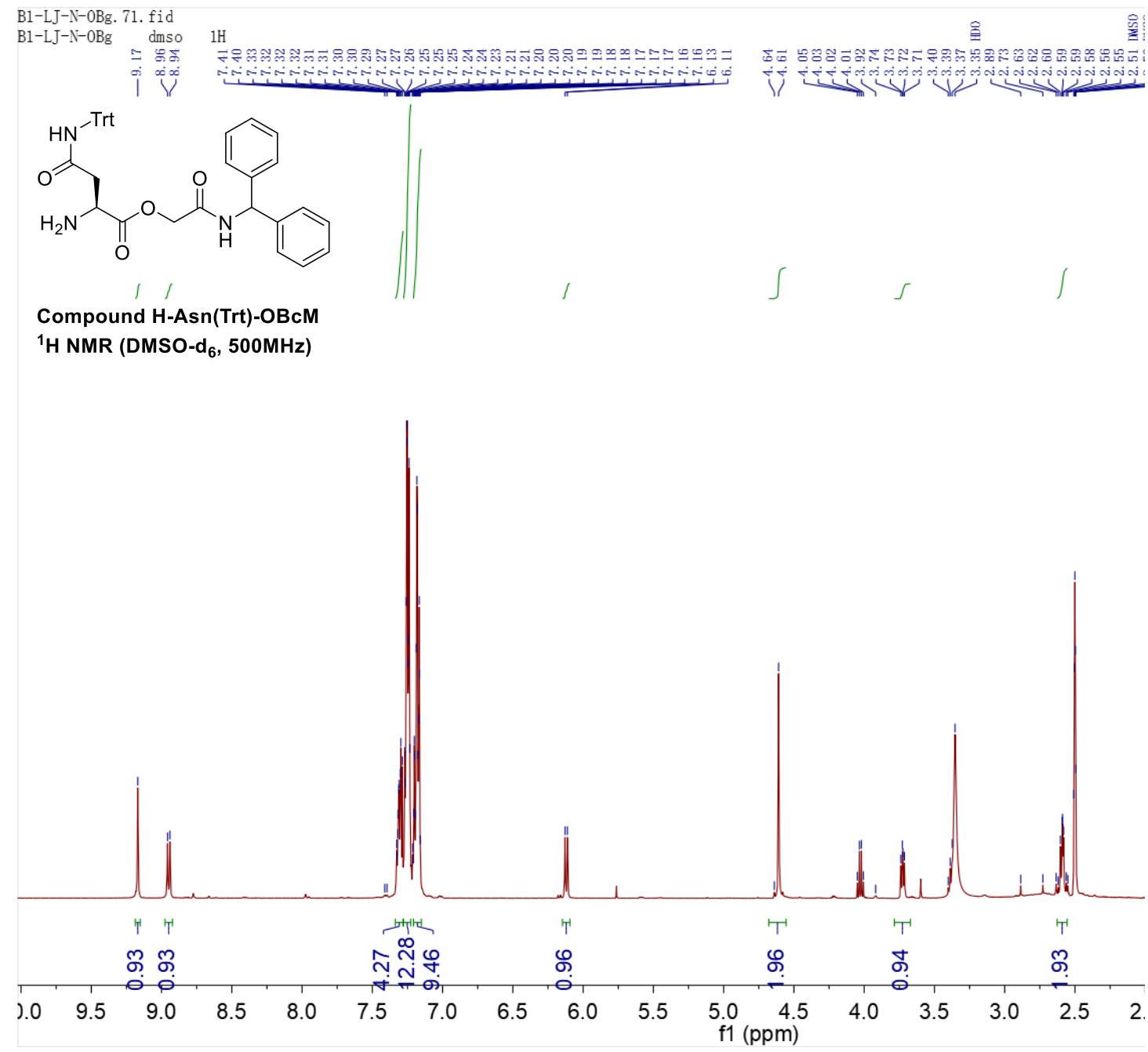

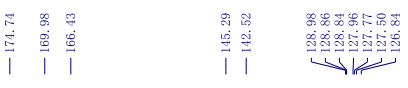

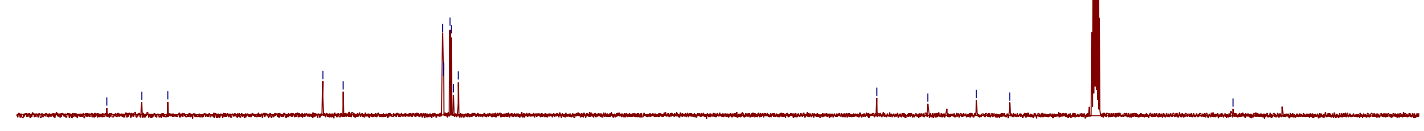

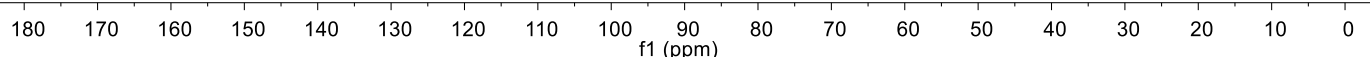




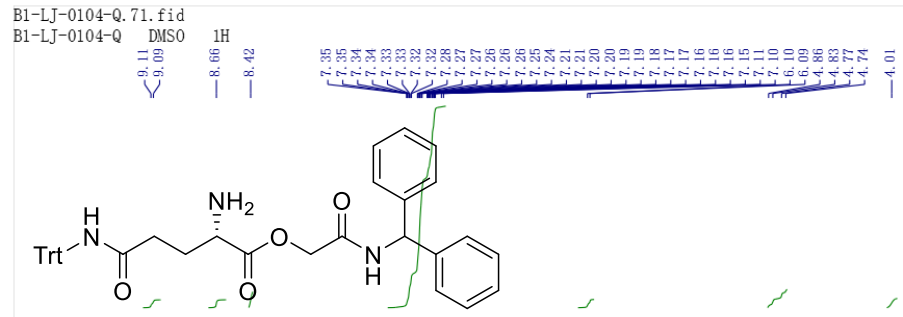

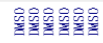

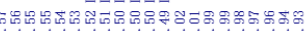

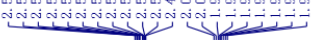

Compound H-GIn(Trt)-OBcM

${ }^{1} \mathrm{H}$ NMR (DMSO-d $6,500 \mathrm{MHz}$ )

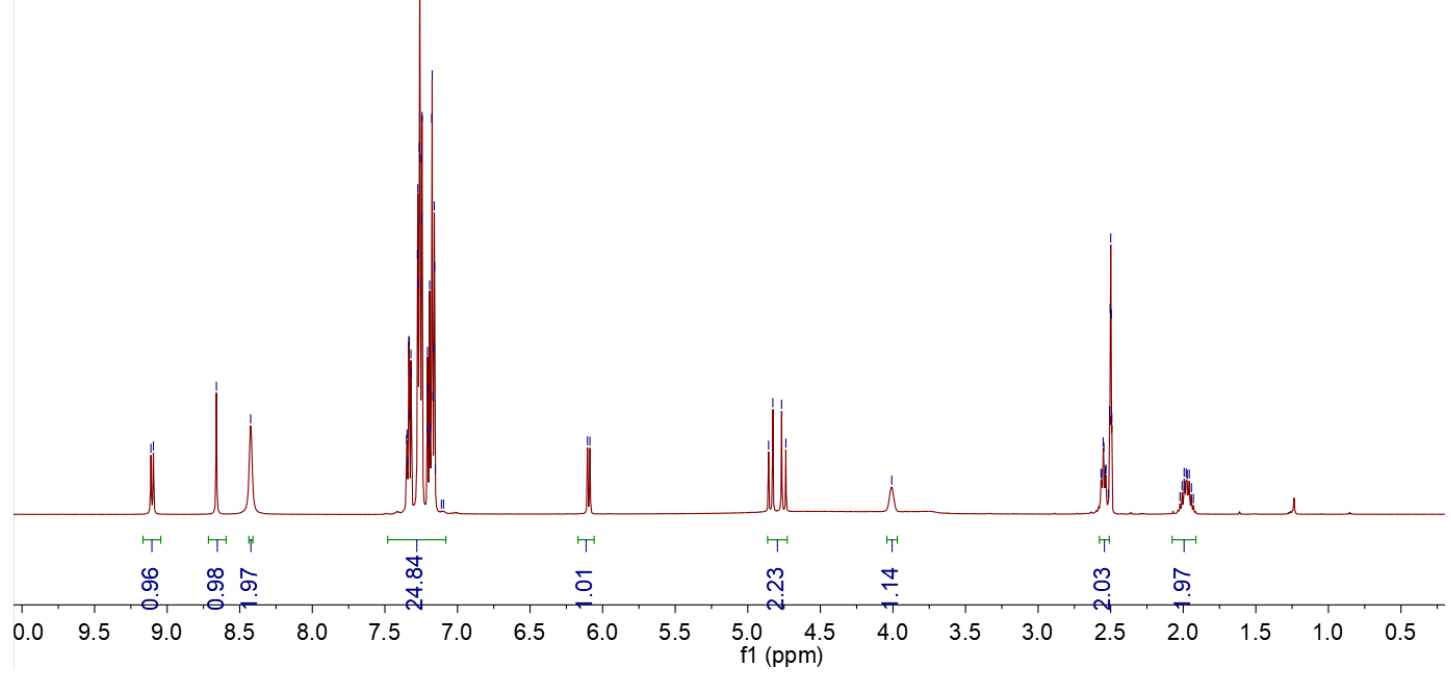

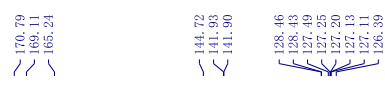
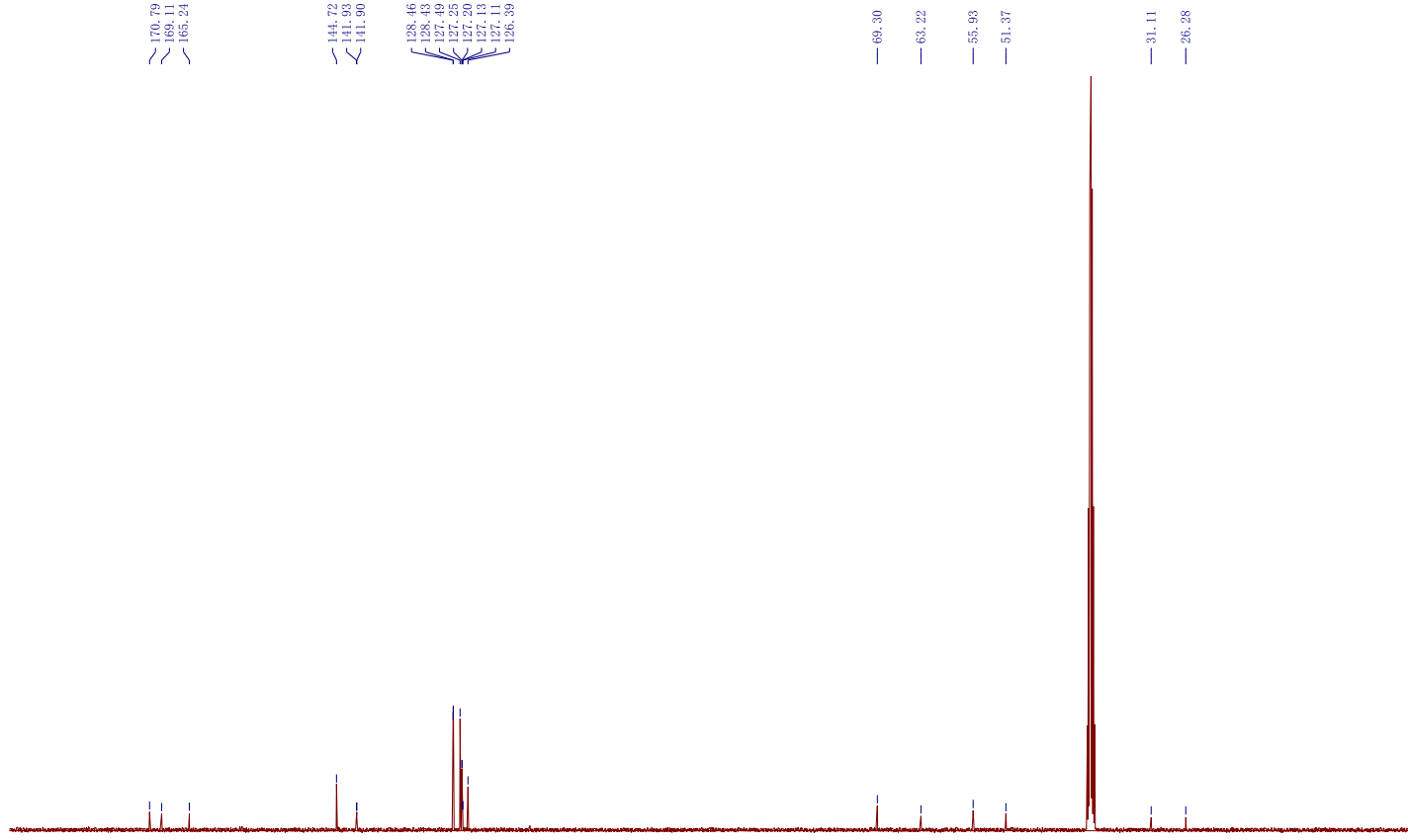

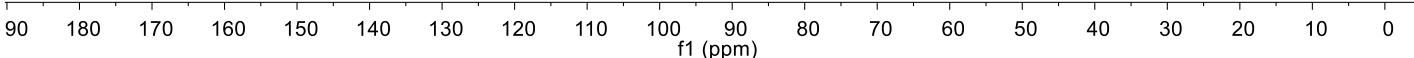




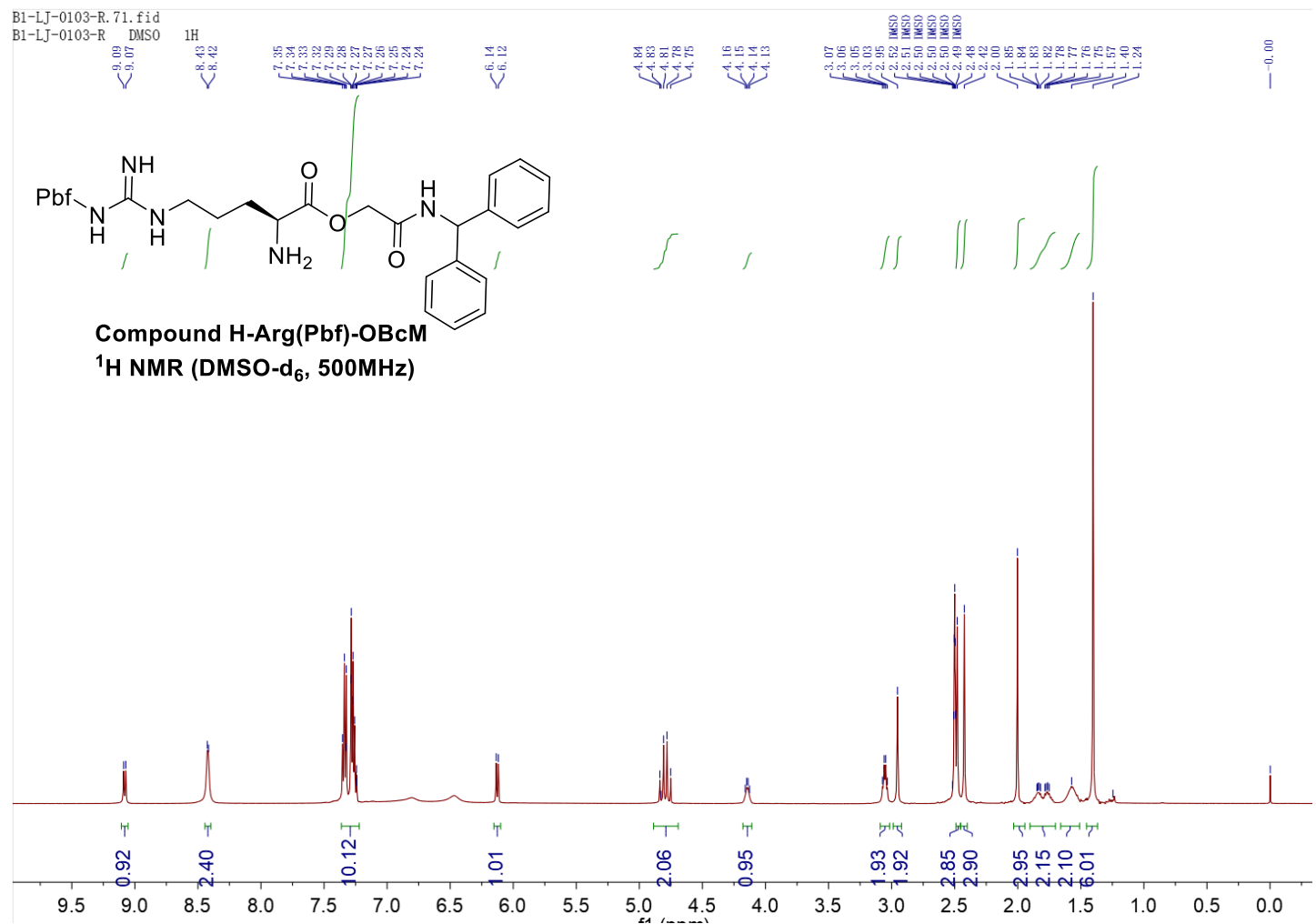

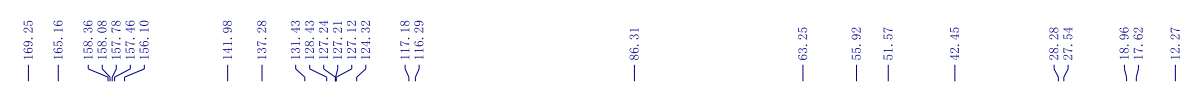

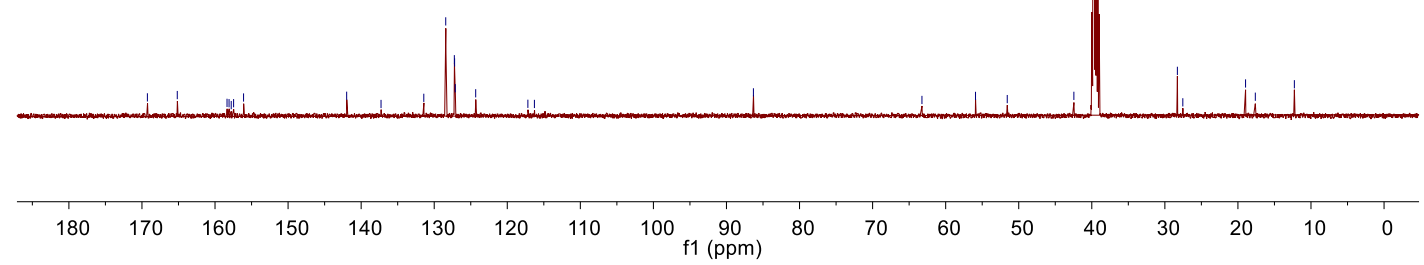



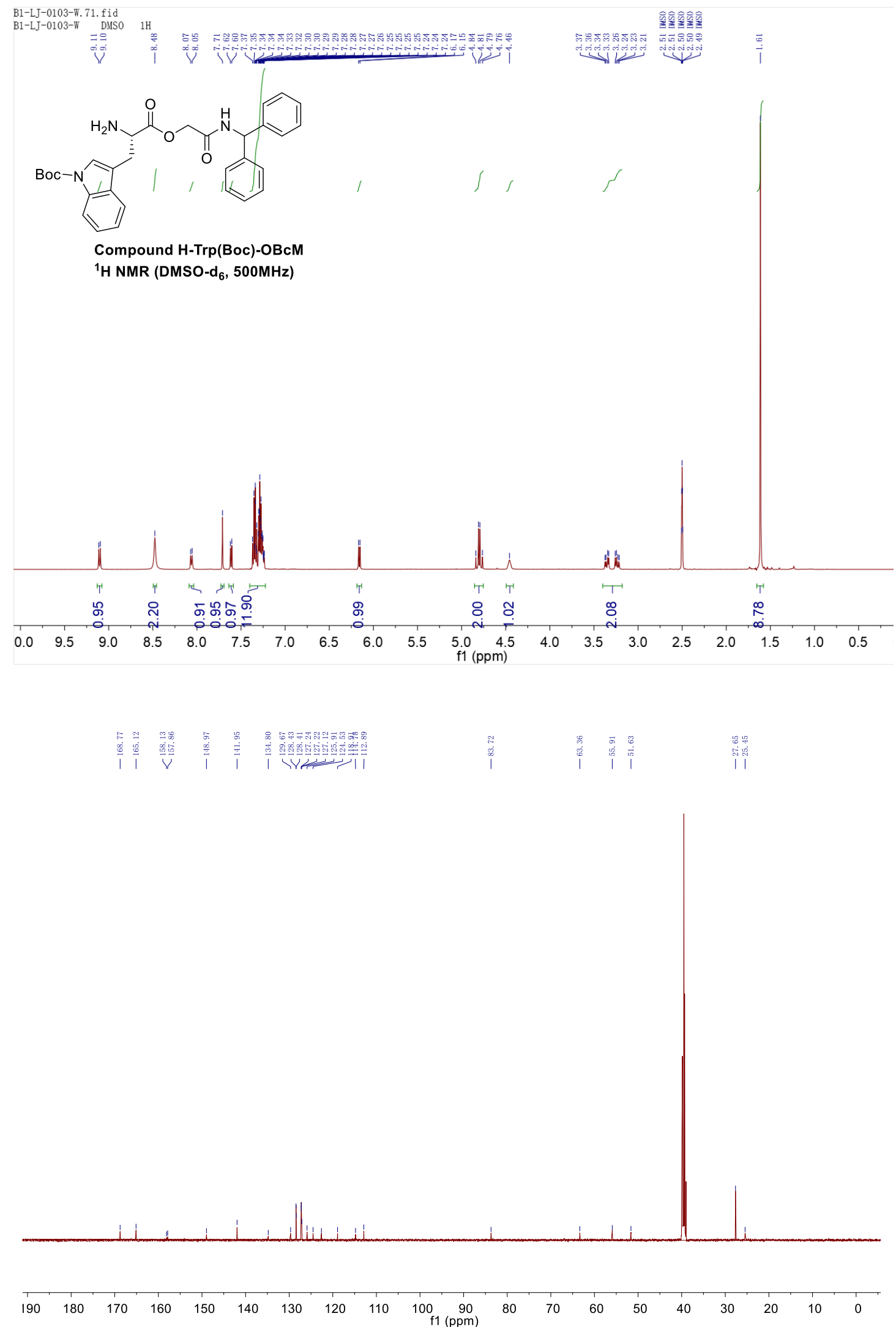

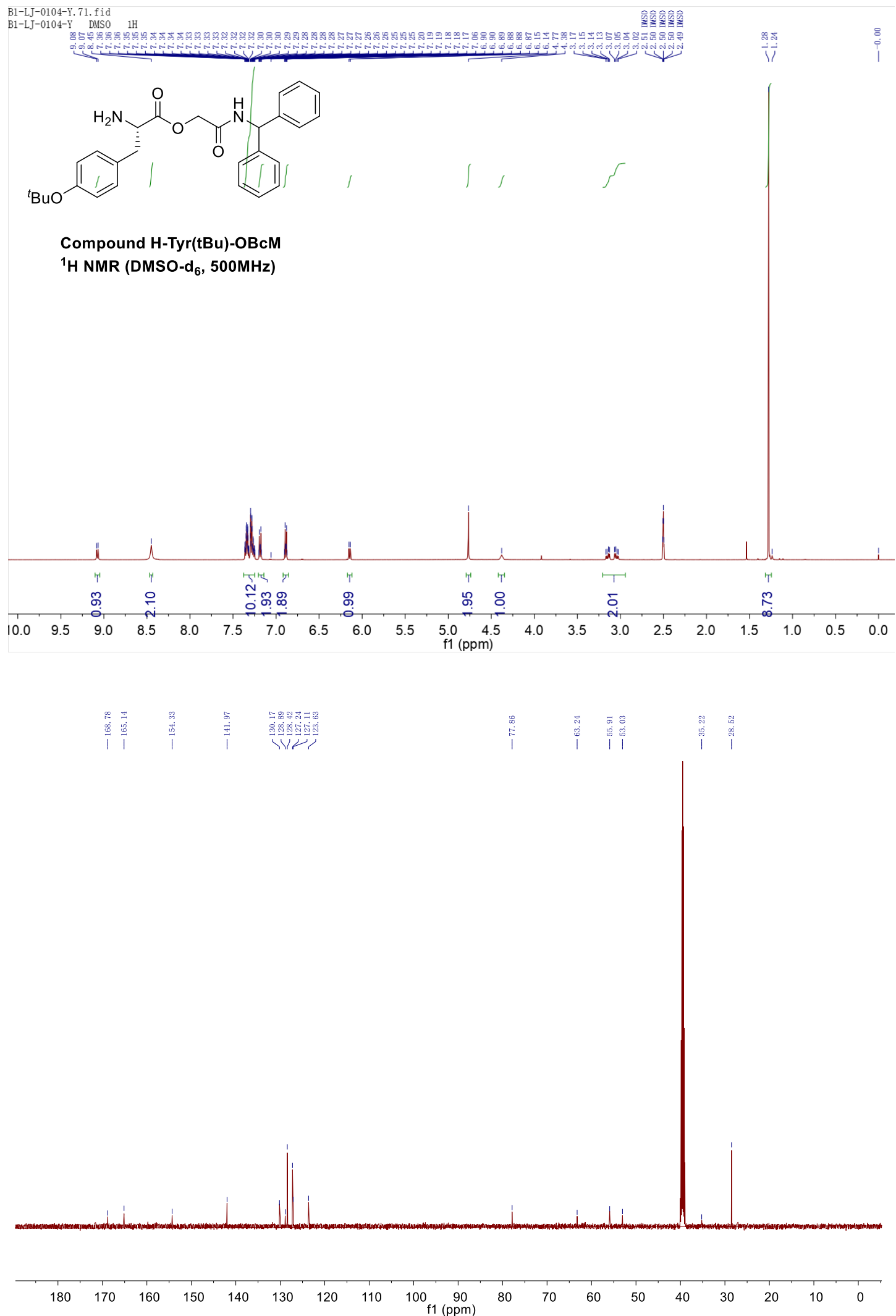


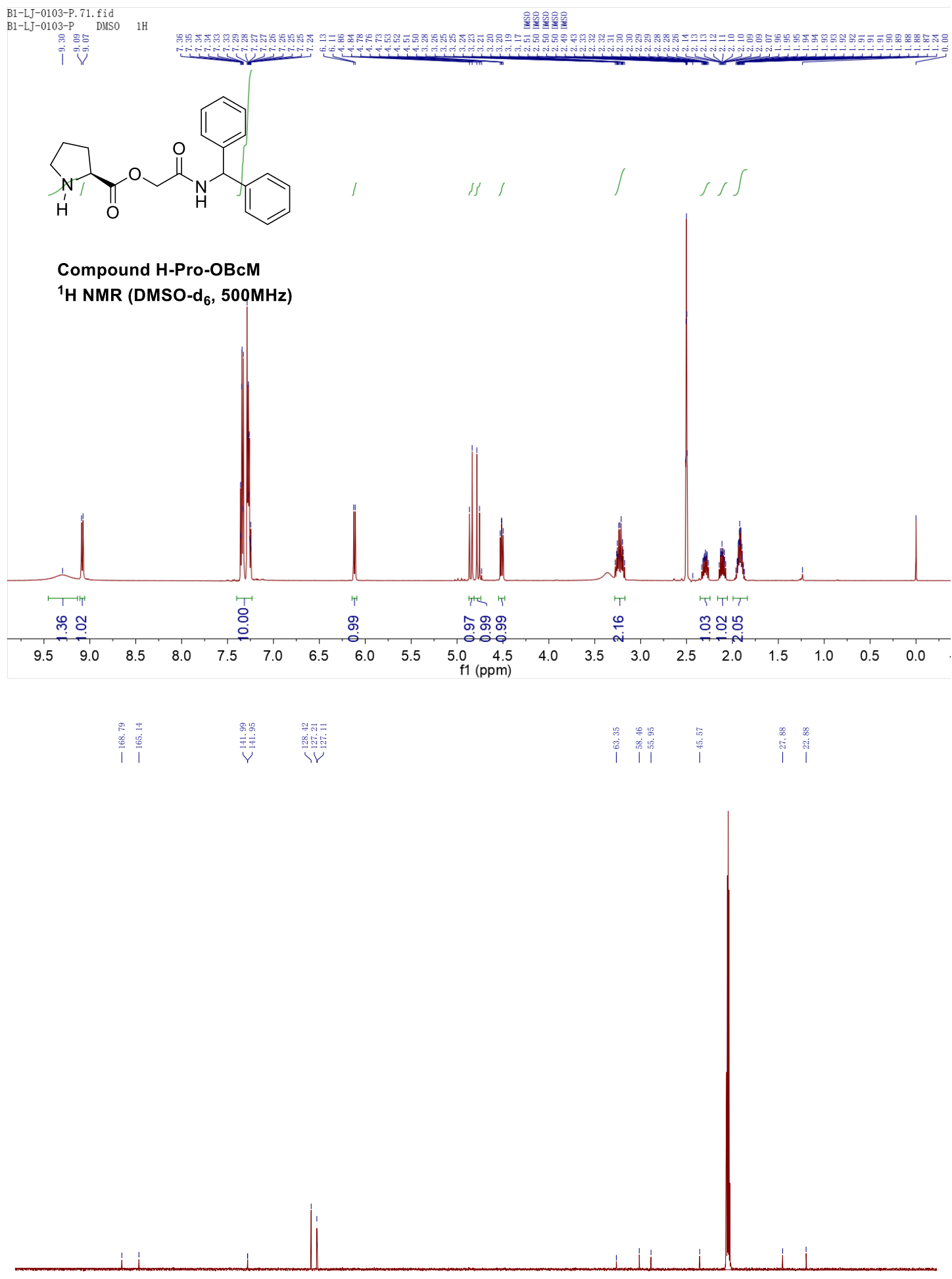

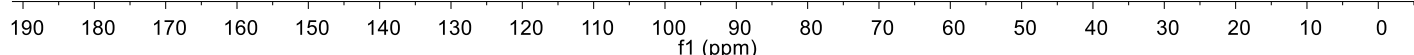



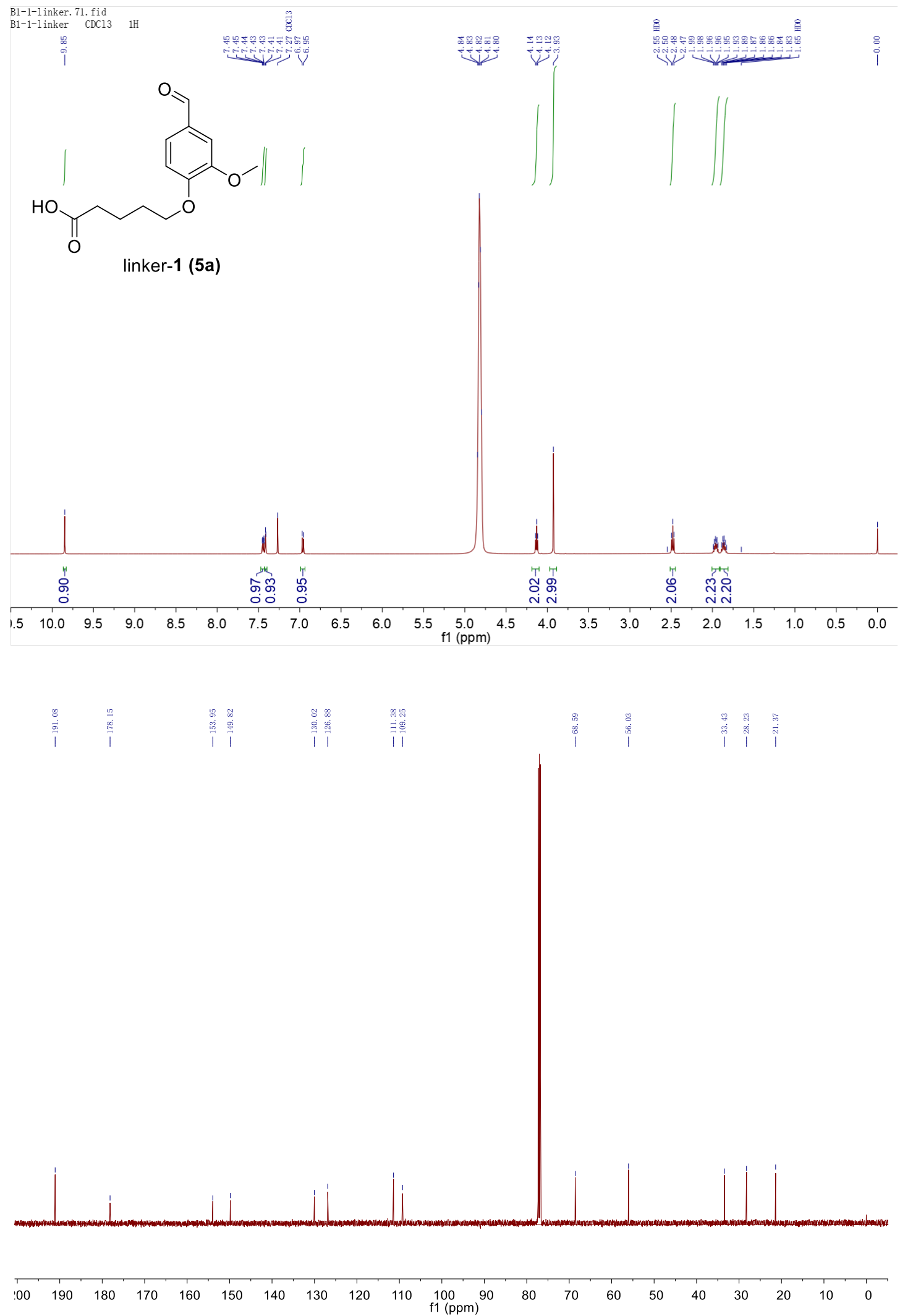

S67 

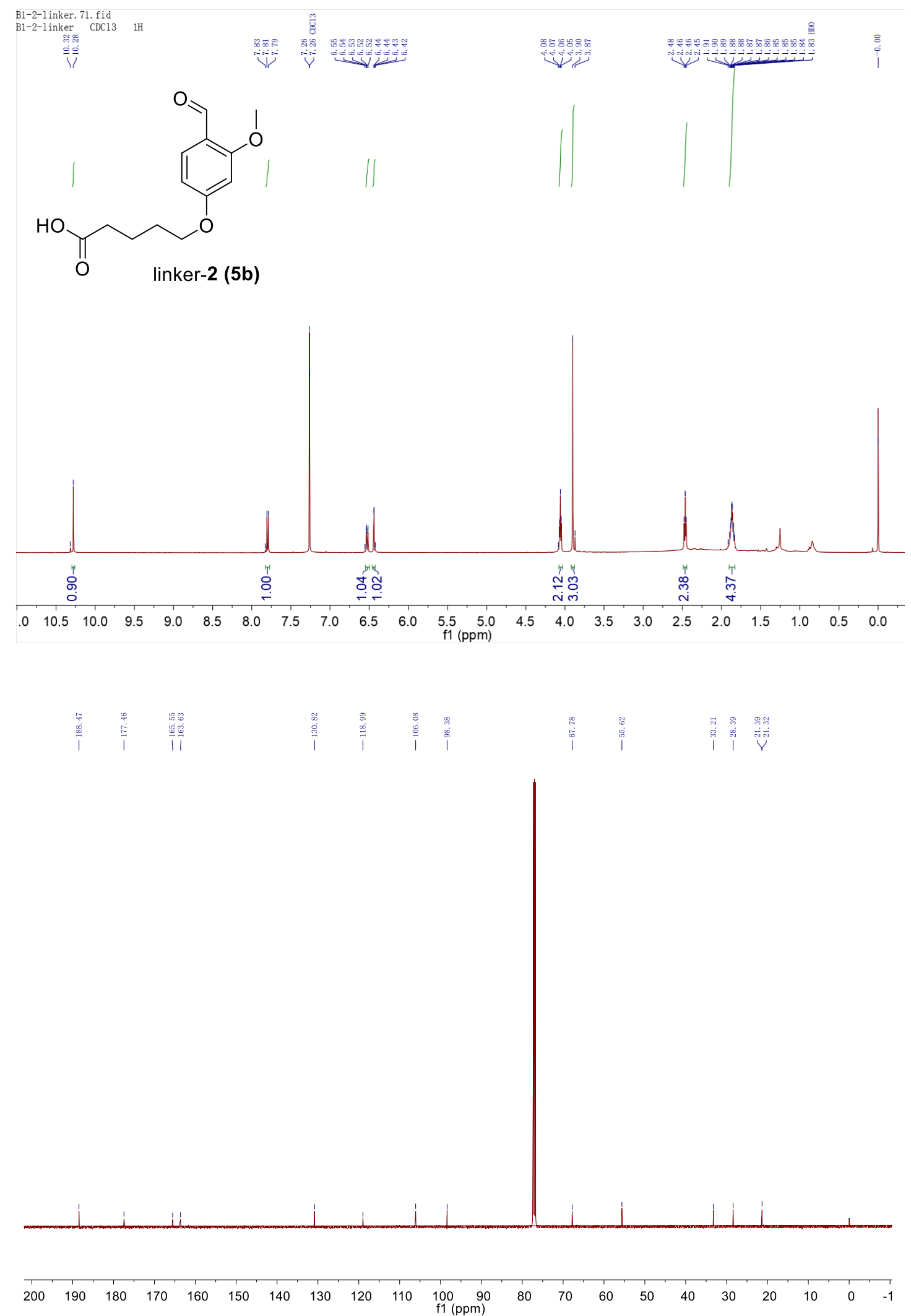

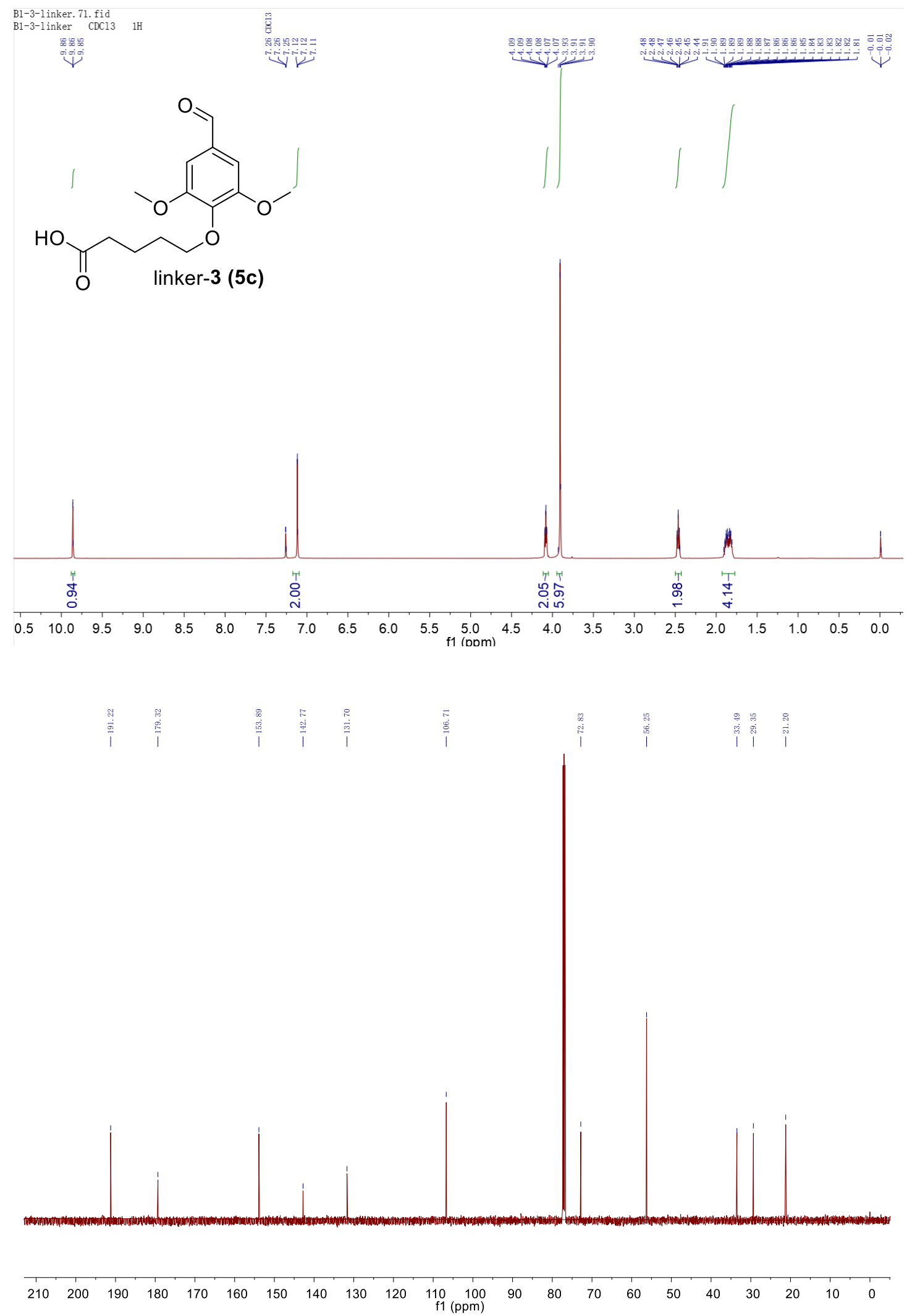


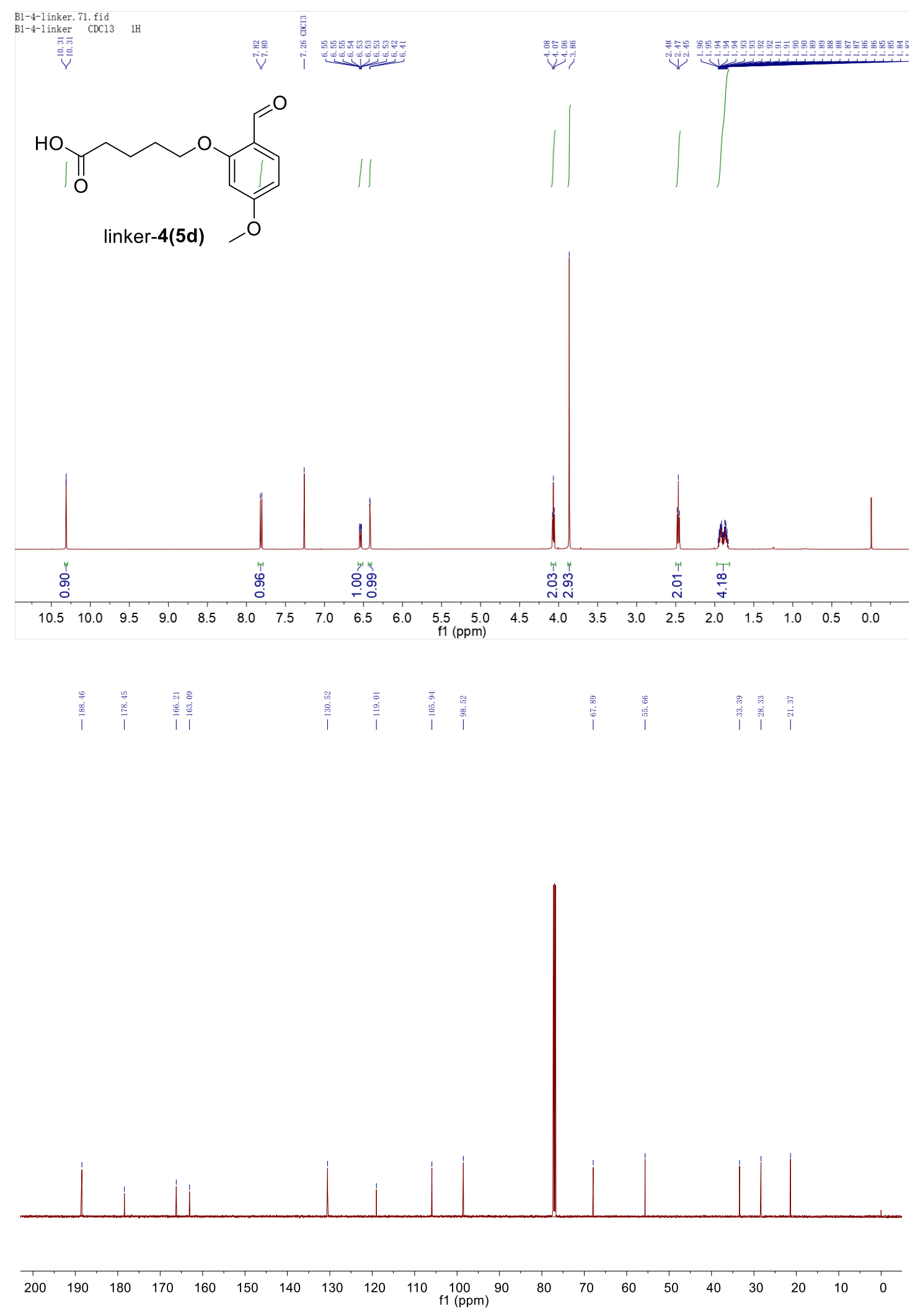




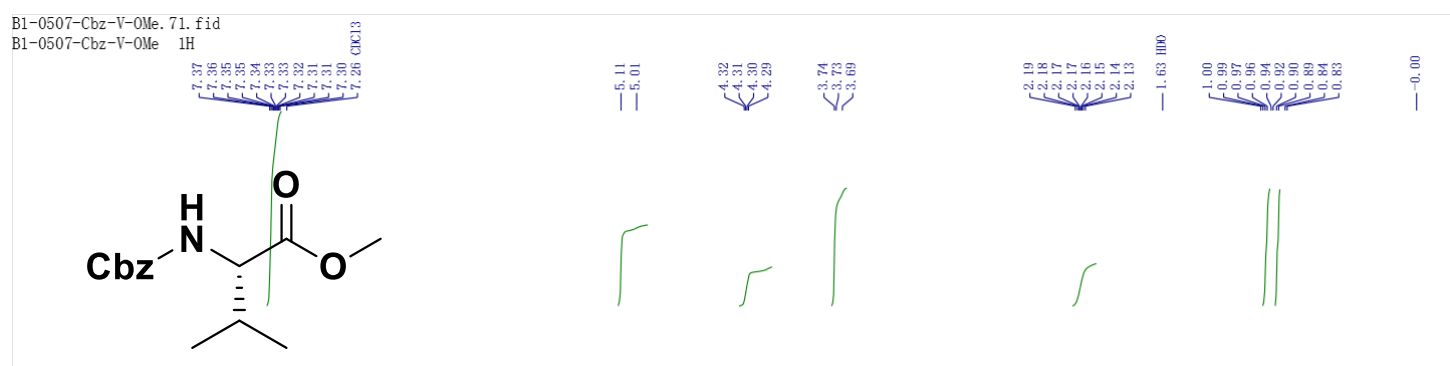

Cbz-V-OMe (3a)
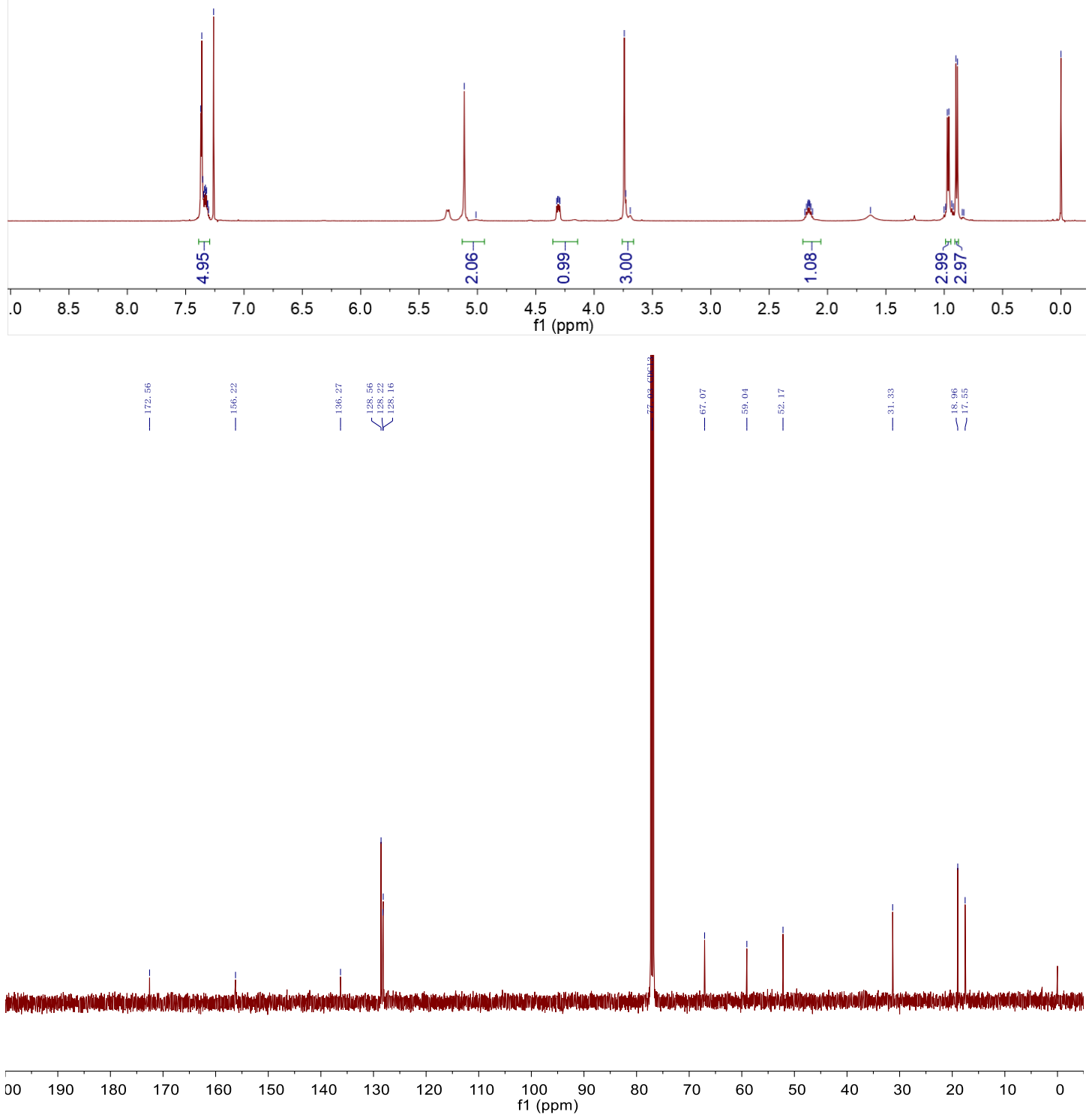
B1-Cbz-V-OEt. 71. fid
B1-Cbz-V-OEt CDC13 $\quad 1 \mathrm{H}$

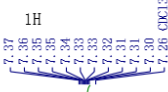<smiles>CCOC(=O)C(NC(=O)OCc1ccccc1)C(C)C</smiles>

\section{Cbz-V-OEt (3b)}

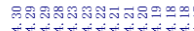

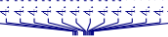

官

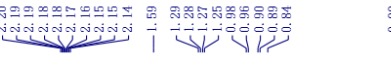

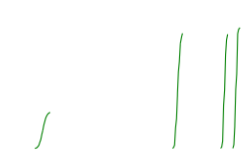




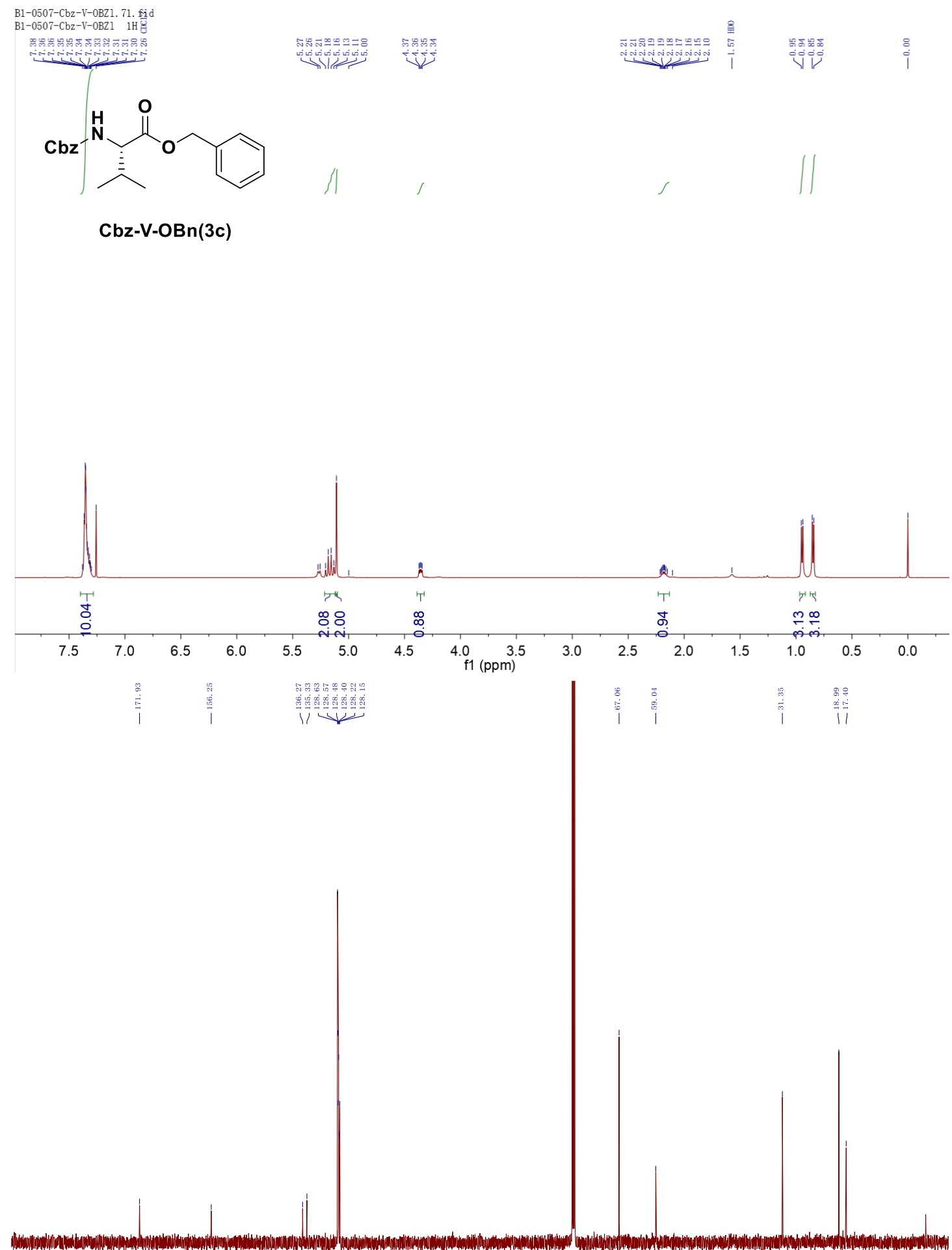

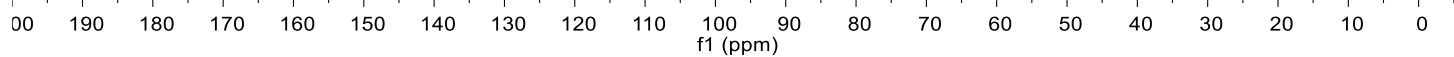



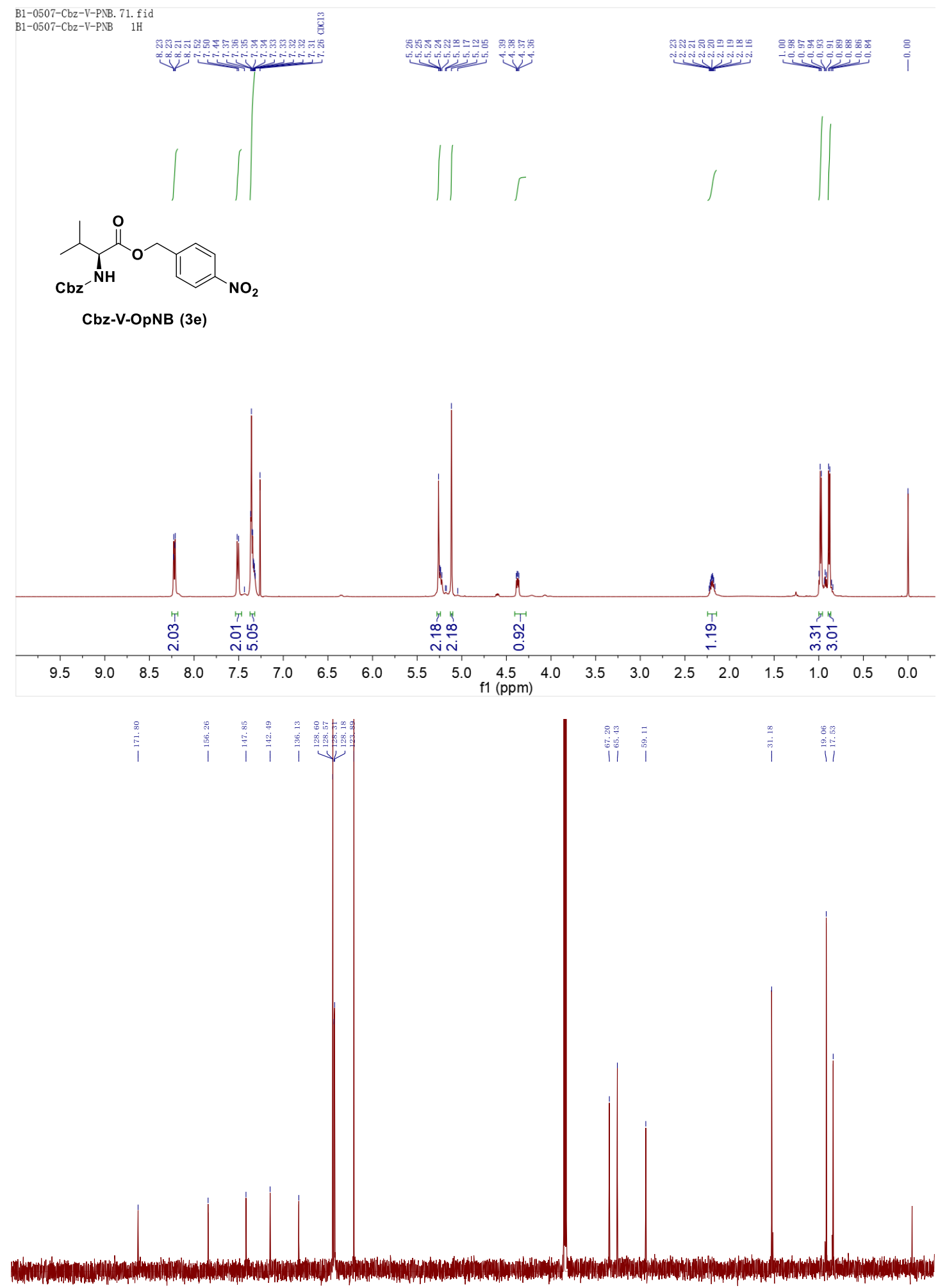

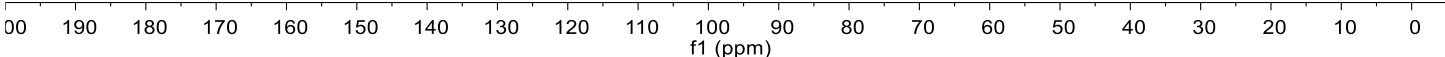




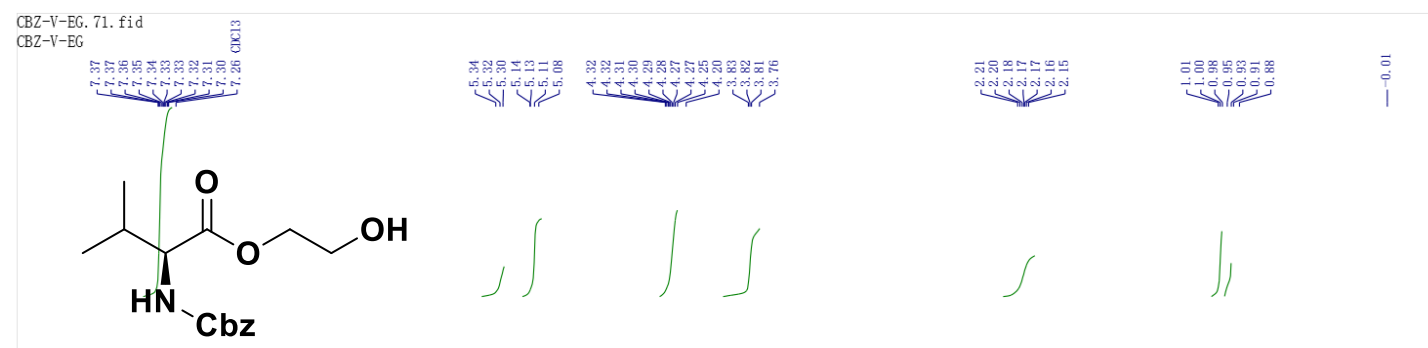

Cbz-V-OEg (3d)
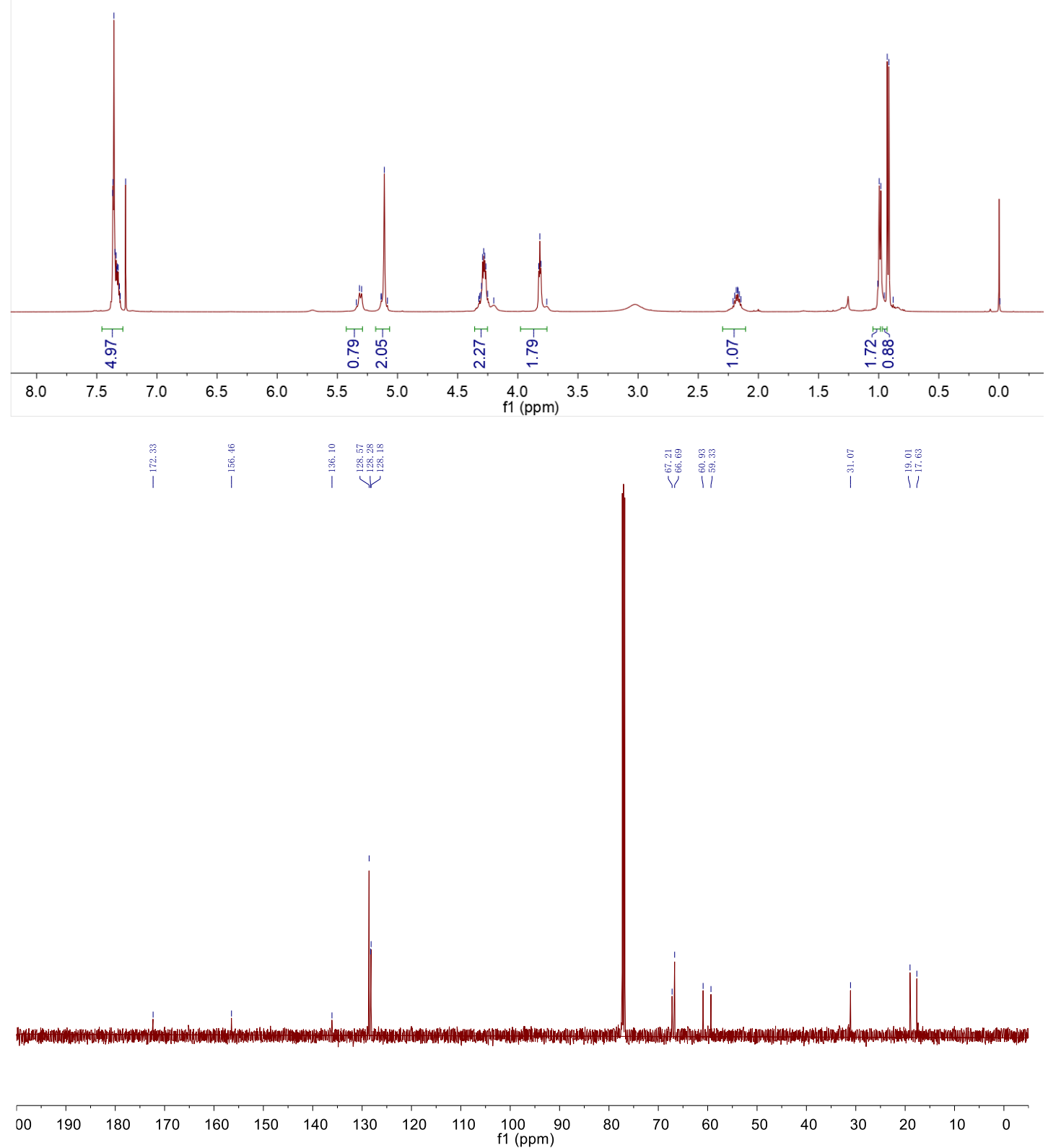


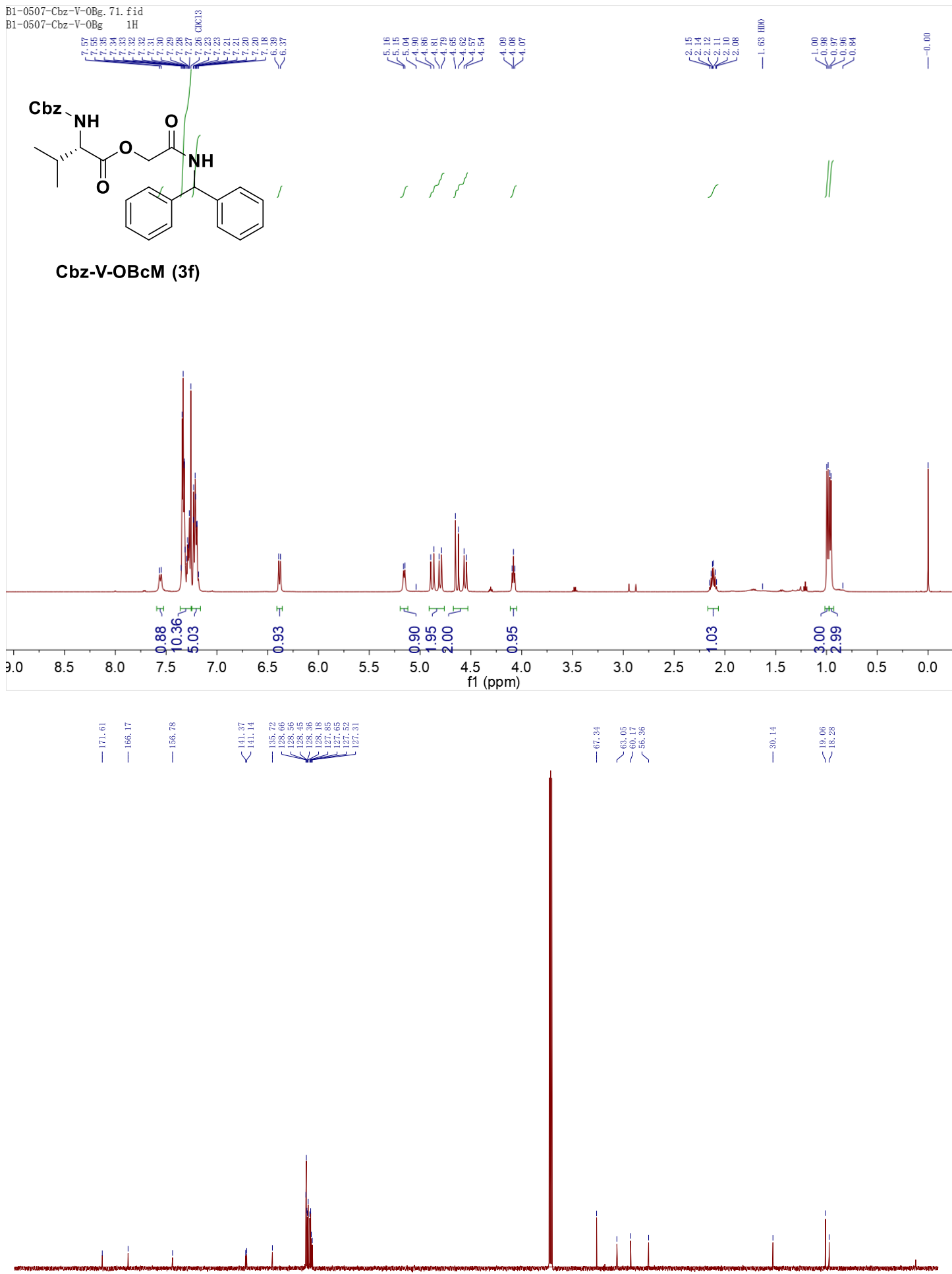

$\begin{array}{lllllllllllllllllll}90 & 180 & 170 & 160 & 150 & 140 & 130 & 120 & 110 & 100 \underset{\mathrm{f} 1(\mathrm{ppm})}{90} & 80 & 70 & 60 & 50 & 40 & 30 & 20 & 10 & 0\end{array}$ 


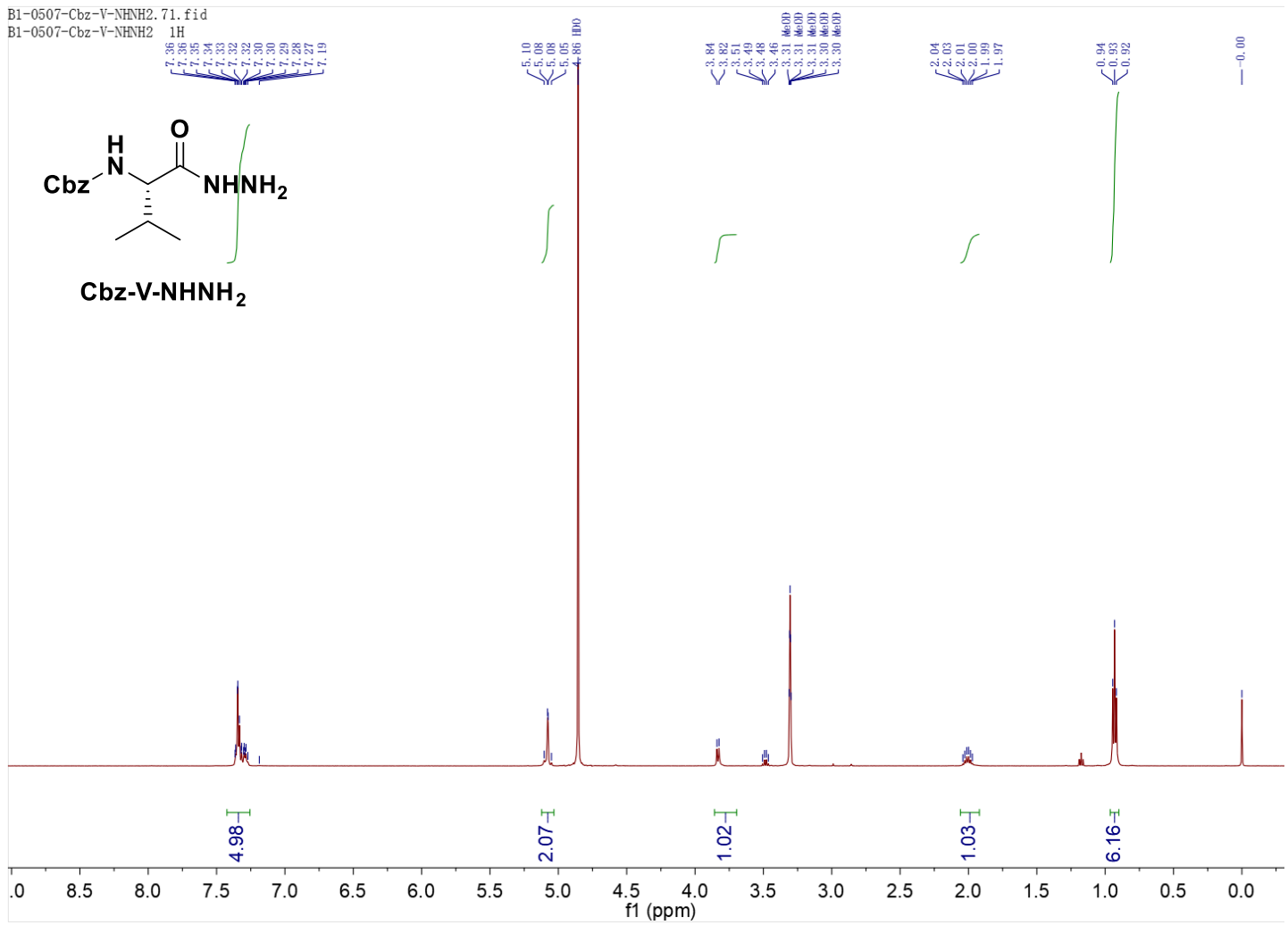

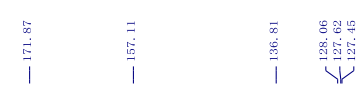

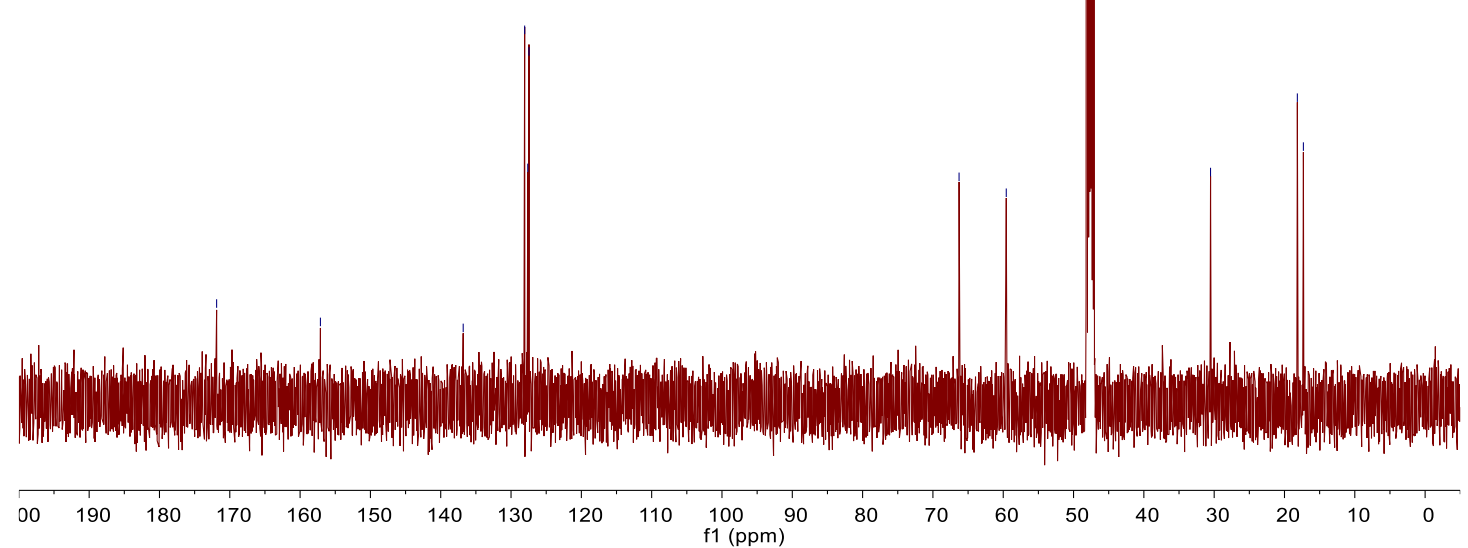




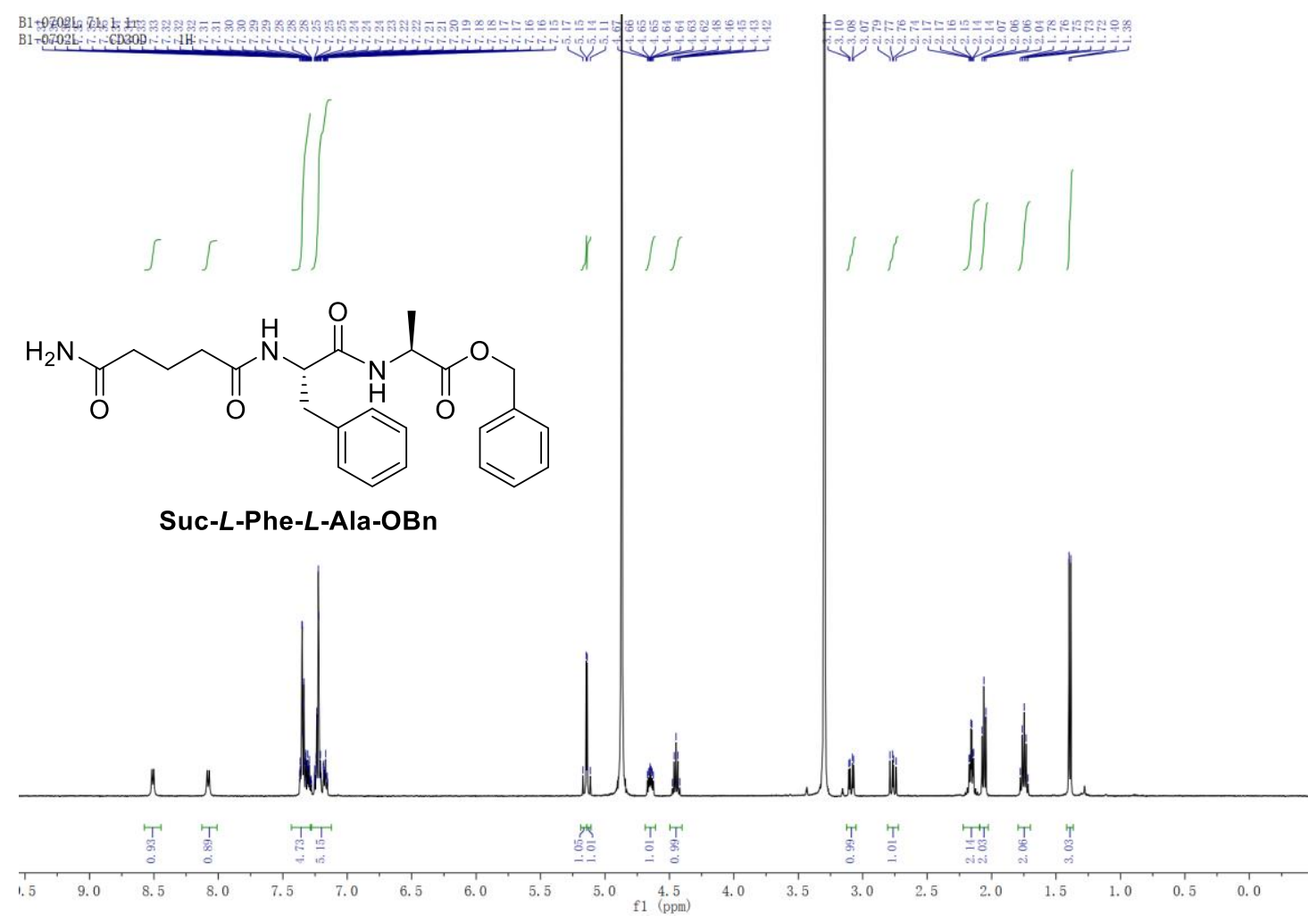

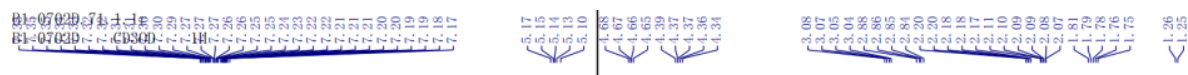
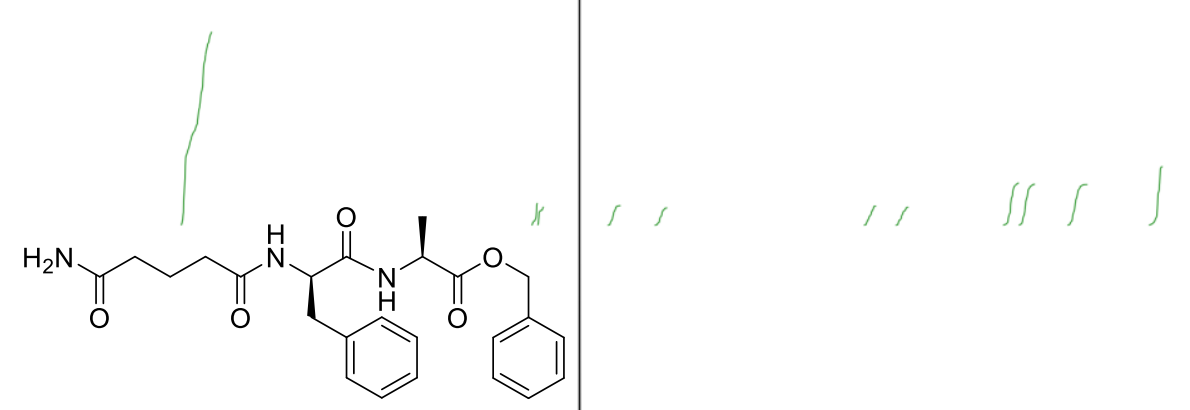

Suc-D-Phe-L-Ala-OBn

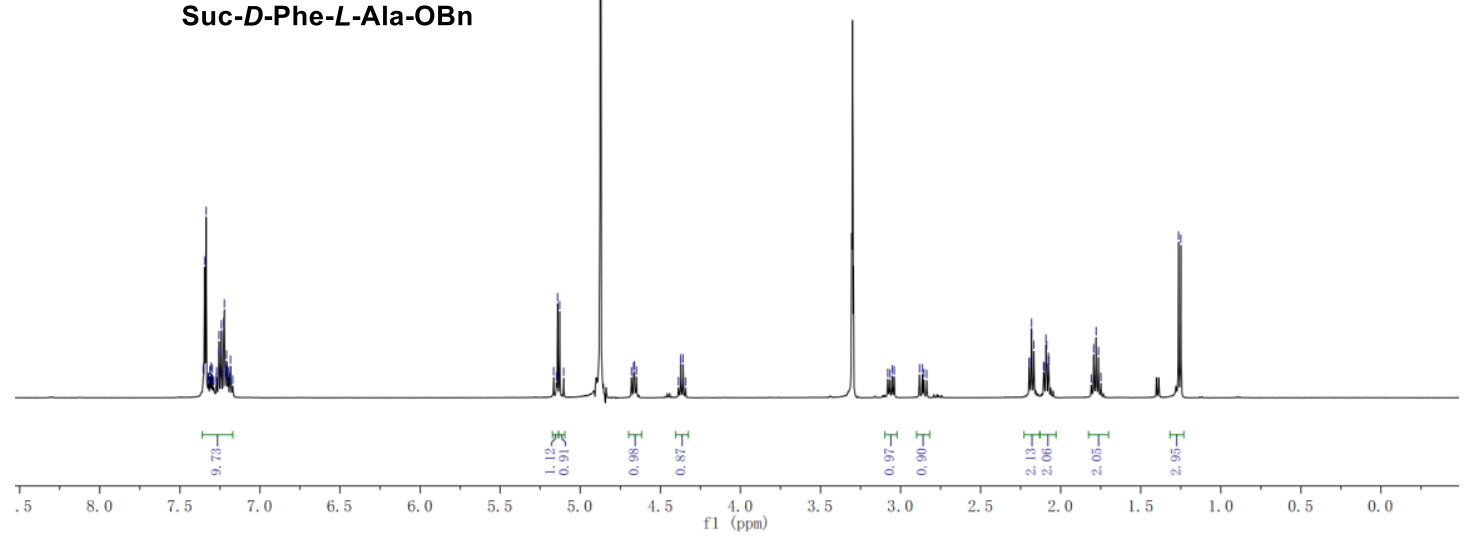

